\title{
Microseismicity during geothermal stimulation at the Ngatamariki geothermal field: New detections via a matched-filter method
}

\author{
Gabriel Matson
}

A thesis submitted to Victoria University of Wellington in fulfilment of the requirements for the degree of Master of Science in Geophysics

School of Geology, Environment and Earth Sciences

Victoria University of Wellington

Wellington, New Zealand

2016 


\section{Declaration of Authorship}

I, Gabriel Matson, declare that this thesis titled, 'Microseismicity during geothermal stimulation at the Ngatamariki geothermal field: New detections via a matchedfilter method' and the work presented in it are my own. I confirm that:

- This work was done wholly or mainly while in candidature for a research degree at this University.

- Where any part of this thesis has previously been submitted for a degree or any other qualification at this University or any other institution, this has been clearly stated.

- Where I have consulted the published work of others, this is always clearly attributed.

- Where I have quoted from the work of others, the source is always given. With the exception of such quotations, this thesis is entirely my own work.

- I have acknowledged all main sources of help.

- Where the thesis is based on work done by myself jointly with others, I have made clear exactly what was done by others and what I have contributed myself.

Signed:

Date: 


\section{Abstract}

The high-temperature, fluid-dominated Ngatamariki geothermal field is located in the central Taupo Volcanic Zone, North Island, New Zealand, and is used to generate electricity via an $82 \mathrm{MW}$ power plant. Injection wells have been in operation since June 2012. During June and July 2012, injection well NM8 was injected with with cold water in order to improve reservoir permeability. Geothermal stimulation and production may trigger microearthquakes by fluid flow through the reservoir. Close clustering of microseismic events' hypocentres relative to the sourcereceiver distance results in many events having similar waveforms. We capitalize on this relationship by using a matched-filter detection method in which highquality seismograms corresponding to a well-recorded earthquake ("templates") are cross-correlated against continuous data to reveal additional earthquakes with similar characteristics. Clustering of the detections' hypocenters also implies that small variations in travel times between two events corresponds to small differences in hypocentral locations, which is the foundation of the double-difference relocation method.

Using an 11 station seismic network, we detect 863 events via cross-correlation of 110 matched-filter templates during the two months stimulation testing. We locate each of these detections using a double-difference relocation method by which events are relocated based on relative travel times. The locatable seismicity delineates: a northern Ngatamariki cluster, a southern Ngatamariki cluster, and a cluster to the south, at the neighboring Rotokawa field. Seismicity in the northern Ngatamariki cluster (522 events) is of greatest interest for this project due to its proximity to well NM8 and temporal signature relative to injection. The seismicity cluster centers around well NM8 at a depth of $2.1 \mathrm{~km}$ below sea level. Events in this cluster extend to up to $2.5 \mathrm{~km}$ from the injection well. An increase in seismicity near NM8 lags behind the onset of injection by $4-8$ days. In contrast, a seismicity-rate decrease coincides with injection shut-in without any time lag. Local magnitudes in this cluster span the range $-0.09 \leq M_{l} \leq 1.66$ with a completeness magnitude of 0.25 . Seismicity within $200 \mathrm{~m}$ of NM8 is induced 
by thermal stresses caused by the difference in temperature between the injectate and the reservoir. Seismicity further than $200 \mathrm{~m}$, but still within this cluster, from NM8 is induced via pore fluid pressure increases from the injected fluid. The coupled mechanism acts on two different length scales and is known as a thermoporoelastic mechanism. The matched-filter detection of microseismic events allows interpretation of extent of injection well stimulation and the relationship between injection and seismicity. 


\section{Acknowledgements}

I would like to express my gratitude for those who have given their continual support for this project.

My most sincere gratitude goes to my supervisors: Martha Savage and John Townend. Martha and John's guidance, insight, and support were invaluable and essential to the direction of this project; their presence each step of the way was most appreciated. I am so thankful for their willingness to bring me onto this project, which has turned out to be a learning experience of a lifetime. They have shown by example what exceptional work and diligent research should look like, giving me something to strive for and continually pursue.

This project was made possible by generous funding from Mighty River Power. I would especially like to thank Steven Sewell, my contact at Mighty River Power, and now fellow graduate student. Steven not only provided me with the data for this project, but he was an important source of insights and ideas. His exceptional experience working at the Ngatamariki geothermal field was of immeasurable help. I would also like to thank Jonathan Clearwater, Irene Wallis, and Jaime Quinao for their welcome discourse and discussion regarding this project's results.

I would like to thank Calum Chamberlain for his guidance and emphatic willingness to help with roadblocks I had throughout the project. Calum provided me with the bulk of the matched-filter codes, which were originally developed by USGS researcher David Shelly, and further developed by Calum. He was always available to help during key times and was open to any question I had - even the seemingly obvious ones. I am grateful for his support.

For her patience and guidance in my dealings with HypoDD, I would like to thank Sandra Bourguignon. I am so appreciative of her kindness and willingness to answer my barrage of questions. In addition, she also happily provided me with data for the original Ngatamariki catalogue.

My sincere thanks go to Holly Godfrey who kindly read and edited numerous drafts of this document. I am so grateful for her insights on writing, style, and figure design. To Tom Wilson, for always being a great office mate and a go-to resource for obscure coding debugging - I am very happy to have had his help. I would also like to thank Dominic Evanzia for his help with Seisan and NonLinLoc. My gratitude also extends to Katrina Jacobs for helping me find a simple and easy way of 
analyzing my catalogue's frequency-magnitude distribution using MapSeis. To my fellow Mighty River Power-funded graduate students, Chet Hopp and Francesco Civillini for being around to bounce ideas off regarding Ngatamariki. Also, many thanks to Chet for helping me get started with HypoDD. I would also like to thank Laura-May Baratin for being patient and welcoming of any question I could possibly have regarding matched-filtering and Matlab. Many thanks to all of my other fellow graduate students for creating a fun and memorable environment.

My sincerest thanks also goes to the Geophysics technical team: Adrian Benson and Aleksandr Beliaev. They have provided an incredible amount of assistance with computing and debugging. I have learned an immense amount from all the queries I came to them with. Most of all I appreciate their willingness and patience to explain the basics of any problem I had.

I must also thank my family back in the California for their love and encouragement, especially my mother Susan, father David, sister Sophie, brother-in-law Jeff, and Aunts Cheri and Melina. 


\section{Contents}

Declaration of Authorship iii

Abstract $\quad$ v

Acknowledgements vii

$\begin{array}{ll}\text { Contents } & \text { ix }\end{array}$

List of Figures $\quad$ xi

List of Tables $\quad$ xiii

Abbreviations $\quad$ xv

Symbols $\quad$ xvii

1 Introduction 1

1.1 Motivation and objectives . . . . . . . . . . . . . . . 1

1.2 Tectonic setting . . . . . . . . . . . . . . . . . 2

1.2.1 Taupo Volcanic Zone . . . . . . . . . . . . . . . 4

1.2.2 The Ngatamariki geothermal field . . . . . . . . . . . 5

2 Background $\quad \mathbf{1 1}$

2.1 Geothermal fields and stimulation . . . . . . . . . . . . . . 11

2.2 Exploration and production at Ngatamariki . . . . . . . . . . . 13

2.2 .1 Injection well NM8 . . . . . . . . . . . . . . . . 16

2.3 Overview of induced seismicity sources . . . . . . . . . . . . 16

2.3.1 Mechanisms of induced seismicity . . . . . . . . . . 16

2.3.2 Locations of induced seismicity . . . . . . . . . . . . . 18

2.3.3 Detection of induced seismicity . . . . . . . . . . 20

3 Data and Methodology 23

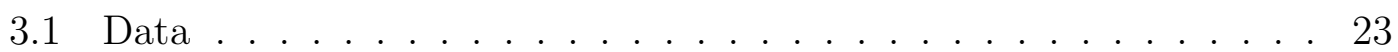


3.1 .1 Seismic data . . . . . . . . . . . . . . . . . 23

3.1 .2 Geothermal field data . . . . . . . . . . . . . . 26

3.2 Cross-correlation theory . . . . . . . . . . . . . . . 27

3.3 Preliminary processing . . . . . . . . . . . . . . . 30

3.3.1 Picking phase arrivals and isolating template events . . . . . 30

3.4 Matched-filter methodology . . . . . . . . . . . . . . . . . 32

3.5 Threshold testing . . . . . . . . . . . . . . . . . 39

3.6 Multiplet construction . . . . . . . . . . . . . . . . . . 43

3.7 Calculating local magnitude using singular value decomposition . . 45

3.7.1 Gutenberg-richter distribution . . . . . . . . . . . . . . . 46

3.8 Double-difference relocation . . . . . . . . . . . . . . . . . 47

4 Results $\quad 53$

4.1 Template multiplets . . . . . . . . . . . . . . . . . 53

4.2 Matched-filter detection . . . . . . . . . . . . . . . . 54

4.3 Double-difference microearthquake locations . . . . . . . . . . . . 62

4.3.1 Uncertainty in earthquake location . . . . . . . . . . . . . 66

4.4 Singular value decomposition magnitudes . . . . . . . . . . . . . . 73

4.5 Diffusion of seismicity . . . . . . . . . . . . . . . . . . 74

$\begin{array}{llr}5 & \text { Discussion } & \mathbf{7 9}\end{array}$

5.1 Induced seismicity f . . . . . . . . . . . . . . . . . . . . . . . . . . . 79

5.1 .1 Seismicity near NM8 . . . . . . . . . . . . . . . . . . . 79

5.1 .2 Seismicity near NM10 . . . . . . . . . . . . . . . . 81

5.1 .3 Seismicity south of Ngatamariki . . . . . . . . . . . . . . 81

5.2 Mechanisms of induced microseismicity . . . . . . . . . . . . . 82

5.2 .1 Thermoelastic stress . . . . . . . . . . . . . . . 83

5.2 .2 Poroelastic stress . . . . . . . . . . . . . . . 85

5.2 .3 Coupled thermo-poroelastic mechanism . . . . . . . . . 86

6 Conclusions $\quad 89$

6.1 Cross-correlation and matched-filtering . . . . . . . . . . . . . 89

6.2 Spatiotemporal analysis of newly detected seismicity . . . . . . . 90

6.3 Mechanisms of induced seismicity . . . . . . . . . . . . . . . . . 91

$\begin{array}{ll}\text { A Matched-filter detection results } & 93\end{array}$

$\begin{array}{ll}\text { Bibliography } & 97\end{array}$ 


\section{List of Figures}

1.1 Tectonic map of New Zealand . . . . . . . . . . . . . . . . . . . 3

1.2 Hikurangi margin cross section . . . . . . . . . . . . . . . . . . 4

1.3 Map of the Taupo Volcanic Zone geothermal fields . . . . . . . . . . 6

1.4 Geologic map of the Ngatamariki geothermal field . . . . . . . . . . 7

1.5 Geologic cross section of the Ngatamariki geothermal field . . . . . 8

1.6 Ngatamaraiki geothermal resource as defined by a magnetotelluric

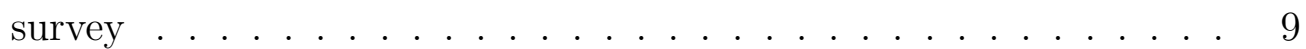

1.7 Hydrologic cross section of the Ngatamariki geothermal field . . . . 10

2.1 Diagram of a geothermal binary power plant . . . . . . . . . . . . 12

2.2 NM8 Injectivity Index scatter plot . . . . . . . . . . . . . . 14

2.3 Timeline for development at the Ngatamariki geothermal field . . . 15

2.4 Example of a diffusion envelope for microseismicity at Fenton Hill, NM. . . . . . . . . . . . . . . . . . . . 18

2.5 STA/LTA methodology schematic . . . . . . . . . . . . . . 21

2.6 Matched-filter methodology schematic . . . . . . . . . . . . . 22

3.1 Map of the Ngatamariki seismic network, injection wells, and tem-

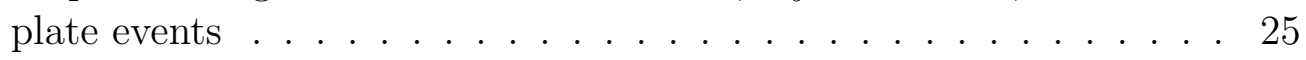

3.2 Drilling fluid loss schematic . . . . . . . . . . . . . . . . . . . . 27

3.3 Selecting a template event window . . . . . . . . . . . . . 31

3.4 Matched-filter methodology flow diagram . . . . . . . . . . . . . . 33

3.5 Example of template waveform . . . . . . . . . . . . . . 34

3.6 Example of correlation sum during 18 June 2012 . . . . . . . . . 36

3.7 Example of a matched-filter detection . . . . . . . . . . . . . . . . 38

3.8 Example of the synthetic testing to determine a matched-filter thresh-

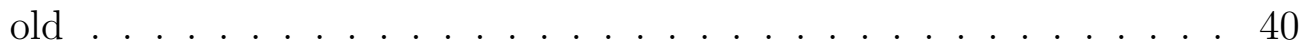

3.9 Results from synthetic testing for determination of matched-filter threshold . . . . . . . . . . . . . . . . . . 42

3.10 Correlation matrix for defining template multiplets . . . . . . . . . 44

3.11 Diagram of the Gutenberg-Richter law . . . . . . . . . . . . . . . 47

3.121 dimmensiional P-wave velocity model for Ngatamariki . . . . . . . 49

3.13 Schematic of the double-difference algorithm . . . . . . . . . . . . . 49

3.14 Example of the capabilities of HypoDD . . . . . . . . . . . . . 50

4.1 Example of two well correlated templates . . . . . . . . . . . . 55 
4.2 Example of two poorly correlated templates . . . . . . . . . . . 56

4.3 Map of template events clustered by multiplet . . . . . . . . . . . 57

4.4 A close look at an interval of high detection rate . . . . . . . . . . . 58

4.5 Detection waveforms from Figure 4.4 . . . . . . . . . . . . . . 59

4.6 Example of stacked detection waveforms for station WPRZ . . . . . 60

4.7 Example of stacked detection waveforms for station NS08 . . . . . . 61

4.8 Scatter plot illustrating the timing of detections for each template . 63

4.9 Scatter plot of total number of detections versus template magnitude 64

4.10 Map of matched-filter detections located using the double-difference relocation method . . . . . . . . . . . . . 65

4.11 Map of matched-filter detections plotted by time . . . . . . . . . . 66

4.12 Enlarged map of seismicity cluster near NM8 . . . . . . . . . . . . 67

4.13 Temporal evolution of seismicity for detections in northern Ngatamariki cluster . . . . . . . . . . . . . . . . 68

4.14 Enlarged map of seismicity cluster near NM10 . . . . . . . . . . . . 69

4.15 Temporal evolution of seismicity for detections in southern Ngata-

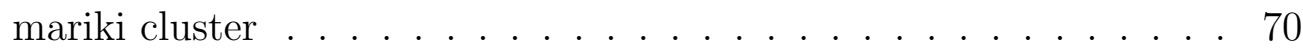

4.16 Enlarged map of seismicity cluster outside of the Ngatamariki geothermal field . . . . . . . . . . . . . . . . . . . 71

4.17 Temporal evolution of seismicity for detections outside the Ngatamariki geothermal field . . . . . . . . . . . . . 72

4.18 Residuals from the double-difference relocation method . . . . . . . 73

4.19 Plot of local magnitude over time . . . . . . . . . . . . . . . . . . . . . . . . . . . . 74

4.20 Histogram of local magnitudes . . . . . . . . . . . . . . . . . . . . . . . . . . . . 75

4.21 Gutenberg-Richter plot of local magnitudes . . . . . . . . . . . . . 76

4.22 Pore fluid pressure diffusion envelope . . . . . . . . . . . . . . . 77

5.1 Diagram of thermal front model . . . . . . . . . . . . . . . . . . 84

5.2 Time evolution of a temperature during injection of cold fluid into a fractured reservoir. . . . . . . . . . . . . . . . 85

5.3 Induced seismicity mechanism diagram . . . . . . . . . . . . . . . . 87

5.4 Mohr circle diagram for mechanisms of induced seismicity . . . . . . 88 


\section{List of Tables}

3.1 Seismic stations around Ngatamariki . . . . . . . . . . . . . 24

3.21 dimmensional P-wave velocity model for Ngatamariki (Zara Rawlinson, pers. com. 2015) . . . . . . . . . . . . . . 48

3.3 HypoDD parameter definitions . . . . . . . . . . . . . . . . 51

3.4 Parameters used for ph2dt . . . . . . . . . . . . . . . 52

3.5 Parameters used for HypoDD earthquake relocation . . . . . . . . . 52

4.1 Double-difference location errors . . . . . . . . . . . . . . . . 72

5.1 Thermoelastic model parameters . . . . . . . . . . . . 84

A.1 Index of all template events and resulting number of detections. Template locations, times, and magnitudes were provided in a data release from Mighty River Power. . . . . . . . . . . . . . . . 93 


\section{Abbreviations}

$\begin{array}{ll}\text { TVZ } & \text { Taupo Volcanic Zone } \\ \text { CVZ } & \text { Central Volcanic Zone } \\ \text { MRP } & \text { Mighty River Power } \\ M_{l} & \text { local magnitude } \\ \text { RT } & \text { seismograph designation at Rotokawa } \\ \text { NS } & \text { seismograph designation at Ngatamariki } \\ \text { NM } & \text { geothermal well designation at Ngatamariki } \\ \text { SNR } & \text { signal-to-noise ration } \\ \text { STA/LTA } & \text { short term-long term average } \\ \text { MAD } & \text { median absolute deviation } \\ \text { SVD } & \text { single value decomposition } \\ \text { LSQR } & \text { least squares } \\ \text { II } & \text { Injectivity Index or injectivity } \\ \text { WHP } & \text { well head pressure }\end{array}$




\section{Symbols}

Chapter 2

$W \quad$ injection flow rate t/hr

WHP well head pressure bar

$P_{r} \quad$ reservoir pressure bar

$\rho_{f} \quad$ fluid density $\mathrm{kg} / \mathrm{m}^{3}$

$g \quad$ acceleration of gravity $\mathrm{m} / \mathrm{s}^{2}$

Z downhole depth m

$K \quad$ coefficient of frictional forces

II Injectivity Index

$t$ time $\mathrm{s}$

$n \quad$ stimulation slope

$\tau \quad$ shear stress $\quad \mathrm{MPa}$

$\sigma_{n} \quad$ normal stress $\quad \mathrm{MPa}$

$\mu_{s} \quad$ coefficient of static friction $\quad \mathrm{MPa}$

$C_{f} \quad$ cohesion $\quad \mathrm{MPa}$

$p_{f} \quad$ pore fluid pressure $\quad \mathrm{MPa}$

$\begin{array}{ll}\sigma_{\text {eff }} & \text { effective normal stress } \\ \mathrm{MPa}\end{array}$

$R \quad$ extent of pore fluid pressure m

D hydraulic diffusivity

Chapter 3, Section 3.2

$g \quad$ template time series

f $\quad$ image time series 


$\begin{array}{clc}\tau & \text { time of cross correlation } & \mathrm{s} \\ M & \text { length of template } & \text { samples } \\ N & \text { length of image } & \text { samples } \\ d_{f, g(\tau)} & \text { Euclidean distance between functions } & - \\ c(\tau) & \text { component of } d_{f, g(\tau)} & - \\ \gamma & \text { normalized correlation coefficient } & \mathrm{m} / \mathrm{s} \\ \bar{g} & \text { template mean } & \mathrm{m} / \mathrm{s} \\ \bar{f}_{\tau} & \text { mean of } f(\tau) \text { in the region under the template } & (\mathrm{m} / \mathrm{s})^{2} \\ e_{f} & \text { energy of zero mean image } & -\end{array}$

Section 3.4

$S \quad$ set of window start times for a template

$m \quad$ number of stations

$n \quad$ number of components per station

ccc day-long vector of $\gamma$ for a station-component

cccSum network sum of day-long vector ccc vectors

$L \quad$ number of station components $(m \times n)$

MAD median absolute deviation

$T \quad$ matched-filter threshold $(R \times \mathrm{MAD})$

$R \quad$ threshold scalar value

$D \quad$ set of detections

$t_{\text {delay }_{i}} \quad$ delay time for station-component $i$

$t_{\text {detection }} \quad$ detection time

$t_{\text {maxccc }_{i}} \quad$ time of max corr. for station-component $i \quad \mathrm{~s}$

$t_{\text {detection phase }_{\text {pick }}} \quad$ phase pick on for detection on station-component $i \quad \mathrm{~s}$

$t_{\text {template phase } \text { pick }_{i}}$ phase pick on for template on station-component $i \quad \mathrm{~s}$

Section 3.7

A matrix of a template's detection waveforms

$U \quad$ matrix of relative amplitudes 
$V \quad$ matrix of basis vectors for the waveforms space

$\Sigma \quad$ matrix of singular values

$K \quad$ kernel matrix

$B \quad$ matrix of relative moments

D $\quad$ zero matrix

Section subsec:Gutenberg-Richter

M local magnitude

$N \quad$ number events with magnitude $M$ or more

$a, b \quad$ constants defining the Gutenberg-Richter law

$M_{c} \quad$ completeness magnitude

Section 3.5

$r \quad$ magnitude of spectrum-amplitude component of $f$

$\phi \quad$ phase component of $f$

radians

$\phi_{\text {rand }}$

randomized phase

radians

$f_{\text {rand }} \quad$ phase randomized noise

$\lambda_{i} \quad$ random scalar for amplitude scaling a template

$\tau_{i} \quad$ random time for seeding template

$g^{\prime}(t) \quad$ scaled template

Section 3.6

mcc mean network cross-correlation coefficient

Section 3.8

$r \quad$ time residual

$t^{o b s}$

observed travel time

$t^{\text {cal }}$

calculated travel time

S

$\delta \mathbf{m}^{k} \quad$ hypocentral perturbation vector

$d r_{i}^{k l} \quad$ difference in residuals at station $i$ between events $k$ and $l$ s

$t_{i}^{k} \quad$ travel time of event $k$ to station $i$ 
$G \quad$ matrix of partial derivatives for double-difference method

d vector of double-differences

$m \quad$ vector of changes in hypocentral parameters

$W \quad$ diagonal matrix of weights 
For my mother, Susan Gerson. 


\section{Chapter 1}

\section{Introduction}

\subsection{Motivation and objectives}

This study aims to characterize seismicity induced by geothermal resource exploitation. Our goal is to use a matched-filter methodology to detect and locate small magnitude earthquakes, or microearthquakes $\left(\leq 3 M_{L}\right)$ within a geothermal field during a time of cold water fluid injection. We use two months of data prior to and after the first stimulation of the Ngatamariki geothermal field to examine the relationship between seismicity and injection.

The North Island of New Zealand sits just west of the Hikurangi Margin where the Pacific Plate subducts westward underneath the Australian Plate (Figure 1.1) (Wilson et al., 1995). Extension from back-arc rifting in the central North Island gives rise to the prominent Taupo Volcanic Zone (TVZ)(Figure 1.2) (Stern et al., 2010, Wilson et al., 1995, Wilson and Rowland, 2016). Volcanism in the TVZ commenced approximately $2 \mathrm{Ma}$ and continues today (Wilson et al., 1995). Starting in the 1950s humans began to exploit the region to produce geothermal energy (Chambefort et al., 2016, Wilson et al., 1995). Continual exploration into the geothermal resources of TVZ resulted in establishing 20 geothermal fields throughout the region (Figure 1.3) (Bibby et al., 1995). The high-temperature, fluid-dominated Ngatamariki geothermal field is used to generate electricity via an 82 MW binary power plant, which was commissioned in 2013 (Chambefort et al., 2014). Due to the reservoir structure of Ngatamariki, injection of fluids back into the deep geothermal aquifer increases the efficiency of power production and sustains the vitality of the resource (Chambefort et al., 2014, Clearwater et al., 2015). 
During June and July 2012, cold water was injected into Ngatamariki injection well NM8 in order to improve permeability through thermal stimulation of the reservoir (Clearwater et al., 2015, Grant et al., 2013). It is well documented that seismicity is often induced from fluid injection related processes, such as thermal contractions and pore fluid pressure increase. Our aim is to apply a matched-filter methodology, a technique that is gaining popularity in seismology (Gibbons and Ringdal, 2006), to create a catalogue of microearthquakes during the stimulation period.

Specific goals for this project include: (1) determine a robust matched-filter threshold, (2) adapt a matched-filter methodology to detect microearthquakes within a background signal, (3) apply a double-difference relocation method to obtain precise relative locations for clusters of seismicity, and (4) use a singular value decomposition method to determine relative magnitudes of events with highly similar waveforms. Upon achieving these goals we will be able to determine the viability of these methods at the Ngatamariki geothermal field and characterize detected microseismicity that occurred during June-July 2012. We aim to determine whether or not seismicity is induced, and if it is, then to determine its mechanism.

\section{$1.2 \quad$ Tectonic setting}

New Zealand sits at the boundary between the Australian and Pacific Plates (Figure 1.1). There are three main areas of interaction between these two plates that are directly relevant to New Zealand: (1) northern subduction along the Hikurangi Margin, (2) the transpressional Alpine Fault, and (3) southern subduction along the Puysegur subduction system. South of the South Island, the Australian Plate subducts towards the northeast beneath the Pacific Plate (Cole and Lewis, 1981, Norris et al., 1990). The plate interface transitions to the dextral, transpressional Alpine Fault, which runs down the length of the South Island. Relative plate motion across the Alpine Fault provides stress to uplift the Southern Alps (Norris et al., 1990). Northward still, the Pacific Plate subducts beneath the Australian Plate along the northern Hikurangi Subduction Zone. Subduction is slightly oblique, with $35 \mathrm{~mm} / \mathrm{yr}$ in the parallel to the margin and $6 \mathrm{~mm} / \mathrm{yr}$ normal to the margin, with a resultant vector of $45 \mathrm{~mm} / \mathrm{yr}$ (Stern et al., 2010). The Hikurangi margin sits east of the North Island, running roughly parallel to the eastern coastline (Figure 1.1) (Cole and Lewis, 1981). 


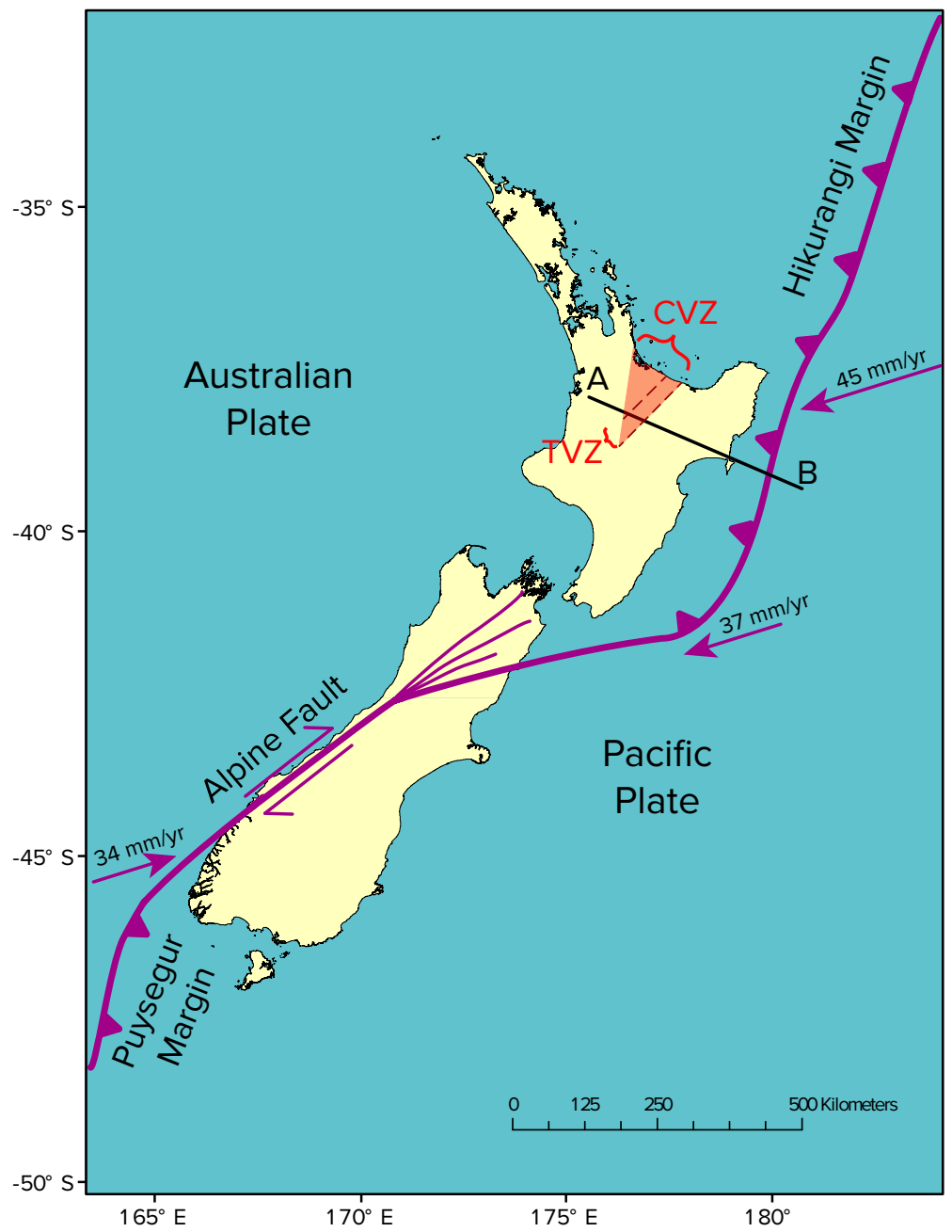

Figure 1.1: Map view of New Zealand Tectonics displaying the Hikurangi Margin, Alpine Fault, and Puysegur Margin. The Central Volcanic Zone (CVZ), TVZ, and cross section trace (A-B)(Figure 1.2) are displayed atop the North Island. Plate motion rates are from Stern et al. (2010).

Oblique subduction of the Pacific Plate underneath the Australian Plate along the Hikurangi Margin causes active volcanism and back-arc rifting (Cole and Lewis, 1981). Volcanism is the result of released water from hydrated minerals within the subducting slab, which lowers the melting point of rocks in the overlying continental lithosphere (Wilson et al., 1995). Accompanying the volcanism is a zone of back-arc extension dominated by northeast trending normal faulting, known as the Central Volcanic Zone (CVZ) (Figure 1.2) (Cole and Lewis, 1981, Stern et al., 2010). 


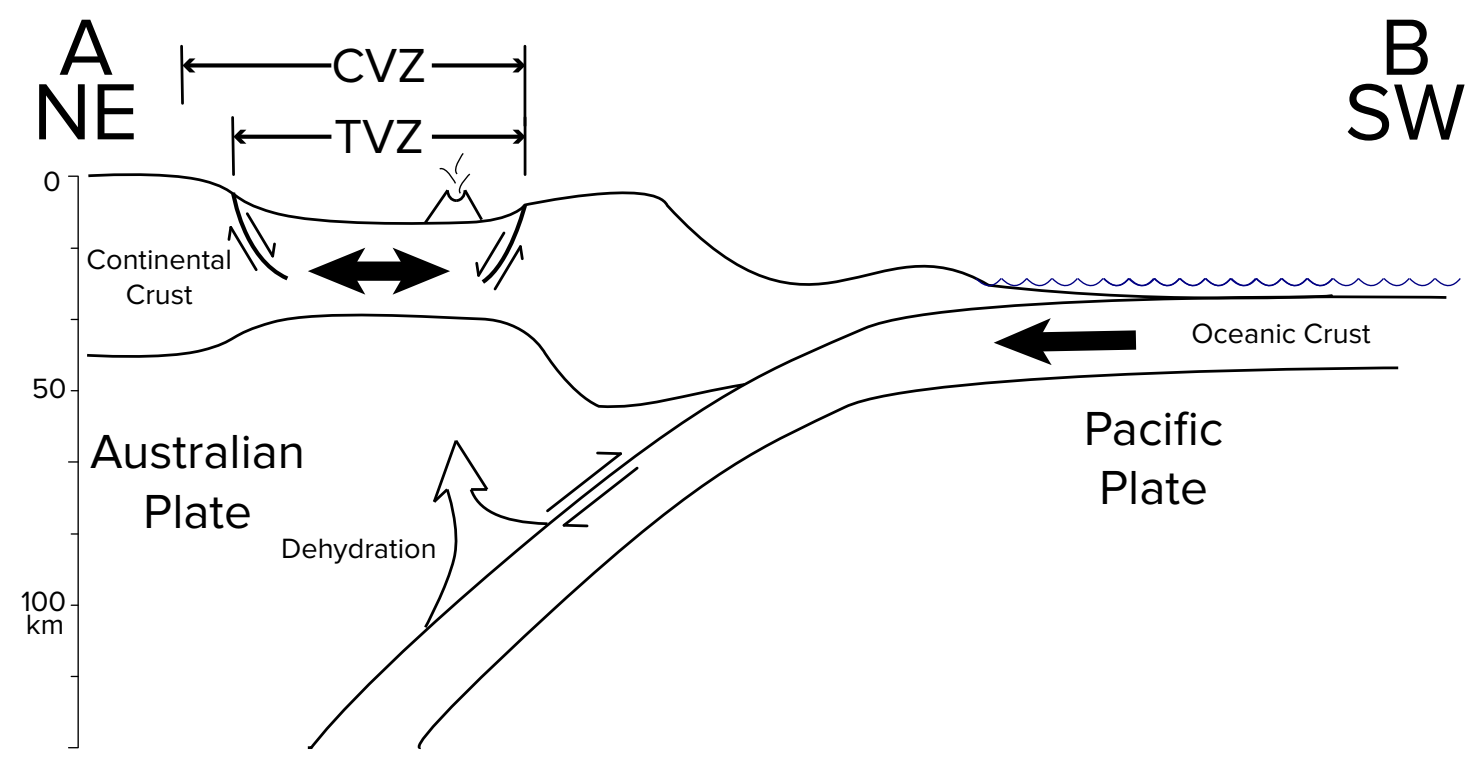

Figure 1.2: Cross section across the Hikurangi margin (A-B) illustrating the back-arc rifting resulting in the extension of the CVZ and TVZ.

\subsubsection{Taupo Volcanic Zone}

The eastern segment of the CVZ wedge is known as the Taupo Volcanic Zone (TVZ) - an area of high heat flow and comprising andesitic to rhyolitic volcanics (Wilson et al., 1995, Wilson and Rowland, 2016). The TVZ is the location for the majority of New Zealands's Pliocene and Quaternary volcanic rocks (Wilson et al., 1995). Early andesitic activity in the area dates to $2 \mathrm{Ma}$, whereas rhyolitic activity commenced later at approximately 1.6 Ma (Wilson et al., 1995, Wilson and Rowland, 2016).

The TVZ is divided into three spatial sections and three temporal sections (Figure 1.3)(Wilson and Rowland, 2016). The Northern Section of the TVZ is dominated by andesite-dacite cones, and extending off shore north of the North Island. The Central TVZ comprises siliceous volcanics, geothermal activity and extensional faulting (Figure 1.3)(Wilson and Rowland, 2016). The Southern TVZ is similar to the Northern TVZ in lithology - primarily andesite-dacite, while the landscape is dominated by large composite cones and minimal hydrothermal activity (Wilson et al., 1995, Wilson and Rowland, 2016).

Wilson and Rowland (2016) describe the three temporal stages in TVZ's evolution. The Old TVZ (2 Ma to $350 \mathrm{ka}$ ) is primarily buried, but outcrops are seen in the western edges of the TVZ and in drill cores. Comprehensive stratigraphy for the 
Old TVZ has not been established (Wilson and Rowland, 2016). Activity during the Young TVZ (350 ka to $61 \mathrm{ka}$ ) accounts for major structural and defining characteristics of the TVZ, the earliest of which was the eruption of the ignimbritic Whakamaru Group. The youngest period of activity is the Modern TVZ (61 ka to present) (Wilson and Rowland, 2016). The Modern TVZ contains features that have great impact on the geothermal activity of the Central TVZ, including frequently active rhyolitic volcanism (Bibby et al., 1995, Wilson and Rowland, 2016).

\subsubsection{The Ngatamariki geothermal field}

The Ngatamariki geothermal field is located northeast of Lake Taupo in the Central TVZ (Figure 1.3). Here we present three main aspects about the field: (1) geology and structure, (2) hydrology, and (3) geothermal features (Figures 1.4, $1.5,1.6$, and 1.7$)$.

Ngatamariki sits atop Torlesse greywacke, a Mesozoic metasedimentary basement rock that was emplaced during subduction (Chambefort et al., 2016, Price et al., 2015, Wilson and Rowland, 2016). The oldest volcanic unit at Ngatamariki is the Reporoa group (1.8 Ma), whose largest member is the Tahorakuri formation (Chambefort et al., 2016, 2014). The Reporoa group comprises all volcanic

and sedimentary formations between the Torlesse Greywacke basement and the Whakamaru Ignimbrites. The Tahorakuri formation includes a 1500 m-thick silicic pyroclastic deposit and overlying lacustrian sediments and tuffs. The lower pyroclastic deposit is defined by three ignimbrite units, all of which have undergone hydrothermal alteration. These ignimbrites are dated at 1.8, 0.9, and $0.7 \mathrm{Ma}$, respectively and together are approximately $900 \mathrm{~m}$ thick. Sedimentary rocks overlaying the ignimbrites are hydrothermally altered, poorly sorted, coarse grained sandstone and carbonaceous lacustrine mudstone/siltstone (Chambefort et al., 2016, 2014).

The 0.4 Ma Whakamaru Group is a 500 m-thick, crystal-rich volcanic deposit erupted from the Whakamaru caldera. This major stratigraphic unit covers Ngatamariki and the entire TVZ (Chambefort et al., 2014). It comprises two units: (1) strongly welded, lithic-poor pumice and (2) non-welded, lithic-rich pumice(Chambefort et al., 2016, 2014). The Whakamaru Group is devoid of hydrothermal alteration (Chambefort et al., 2016). 


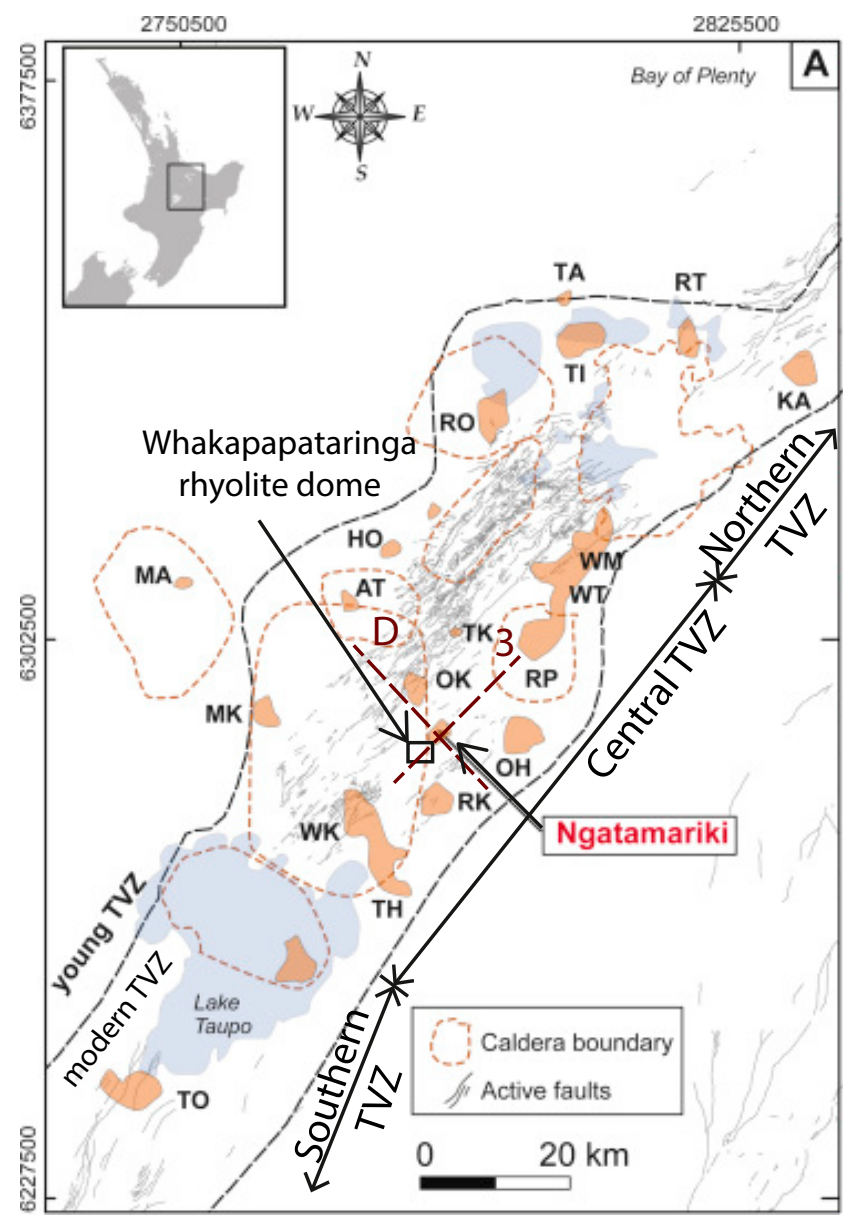

Figure 1.3: An overview of the geothermal fields of the TVZ (Chambefort et al., 2016, adapted). Each geothermal field of the TVZ is highlighted in orange. Ngatamariki sits in the Central TVZ northeast of Lake Taupo. The cross-sections D and 3 are illustrated in Figure 1.6.

Overlying the Whakamaru group are three younger units: the Waiora, Huka Falls, and Oruanui formations (Chambefort et al., 2016). The 0.25 Ma Waiora Formation is an interbedded tuff overlain by rhyolitic lavas of variable thickness that is interbedded with fine-grained, dark siltstone (Chambefort et al., 2016, 2014). The Huka Falls formation is a sequence of lacustrine mudstone, siltstone and tephra formed 0.25 Ma. The Oruanui formation is an even younger surficial deposit of reworked material (Chambefort et al., 2016, 2014).

Ngatamariki is unique among TVZ geothermal fields due to the presence of an intrusive complex (Chambefort et al., 2016, Wilson and Rowland, 2016) The intrusive bodies reach depths as shallow as 2000 meters below sea level (mbsl) (Chambefort et al., 2016, 2014). The complex includes three bodies ranging in age from 0.55-0.72 Ma. The two shallowest units are microdiorite and quartz diorite. The microdiorite has a uranium-lead (U-Pb) date of $0.72 \mathrm{Ma}$, whereas the 


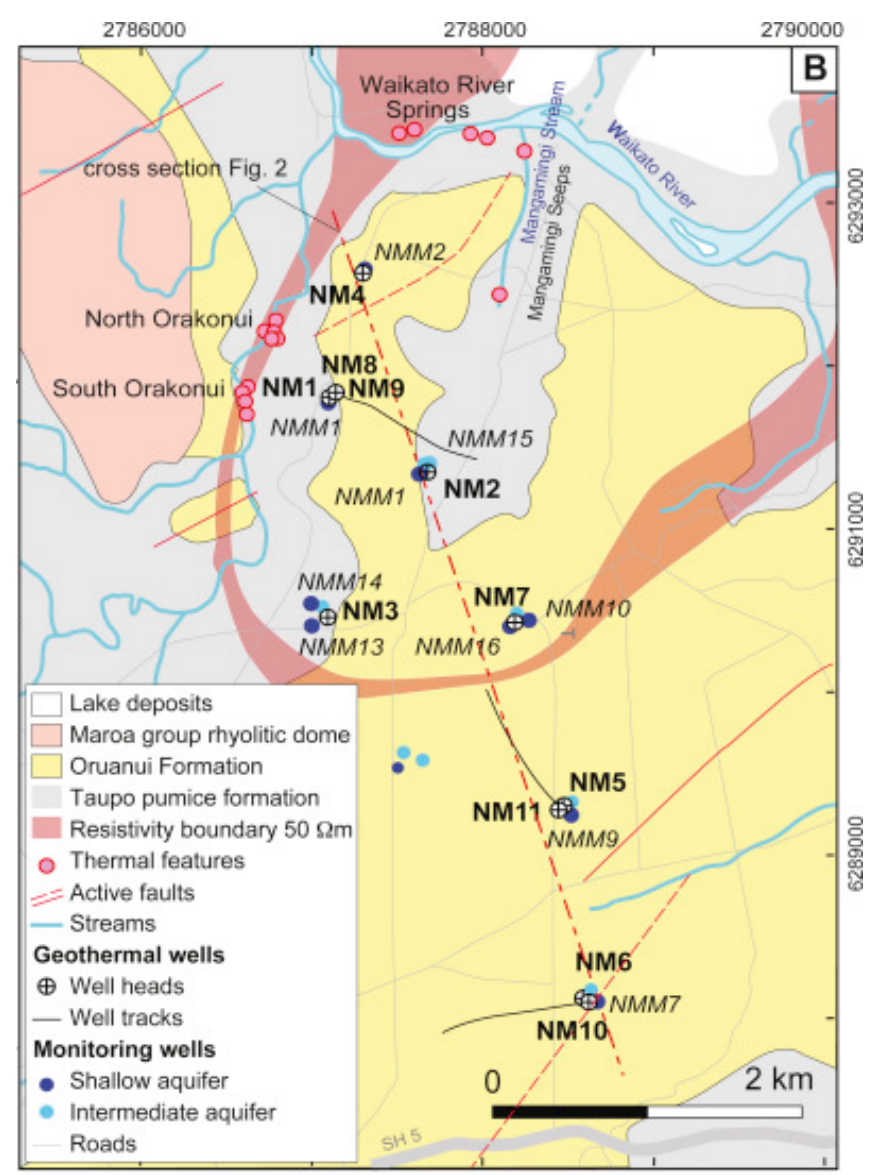

FiguRE 1.4: An overview geologic map of the Ngatamariki geothermal field depicting geothermal wells, active faults, and volcanic features (Chambefort et al., 2016). The cross-section marked with the dashed line is illustrated in Figures 1.5 and 1.7.

quartz diorite has been dated with an argon-argon (Ar-Ar) method to be $0.55 \mathrm{Ma}$ (Chambefort et al., 2014). The deepest intrusive body is a tonalite that is dated at 0.66 Ma (Chambefort et al., 2014).

The intrusive complex is inferred to have provided a heat source for now relict hydrothermal alteration during emplacement (0.70-0.55 Ma). Arehart et al. (2002) describe the intrusive complex's presence as evidence for a paleo-geothermal system. There is a lack of alteration of Whakamaru Ignimbrite compared to the upper Reparoa Group, implying the diorite's produced heat had decreased by 330 ka (Arehart et al., 2002, Bertrand et al., 2015, Chambefort et al., 2016). As an active system, the diorite lasted at most $250 \mathrm{kyr}$ : $550 \mathrm{ka}$ (intrusion age) to 330ka (Whakamaru Group age) (Arehart et al., 2002, Milicich et al., 2014). This is evidence disproving the hypothesis that this intrusive complex is the heat source for 


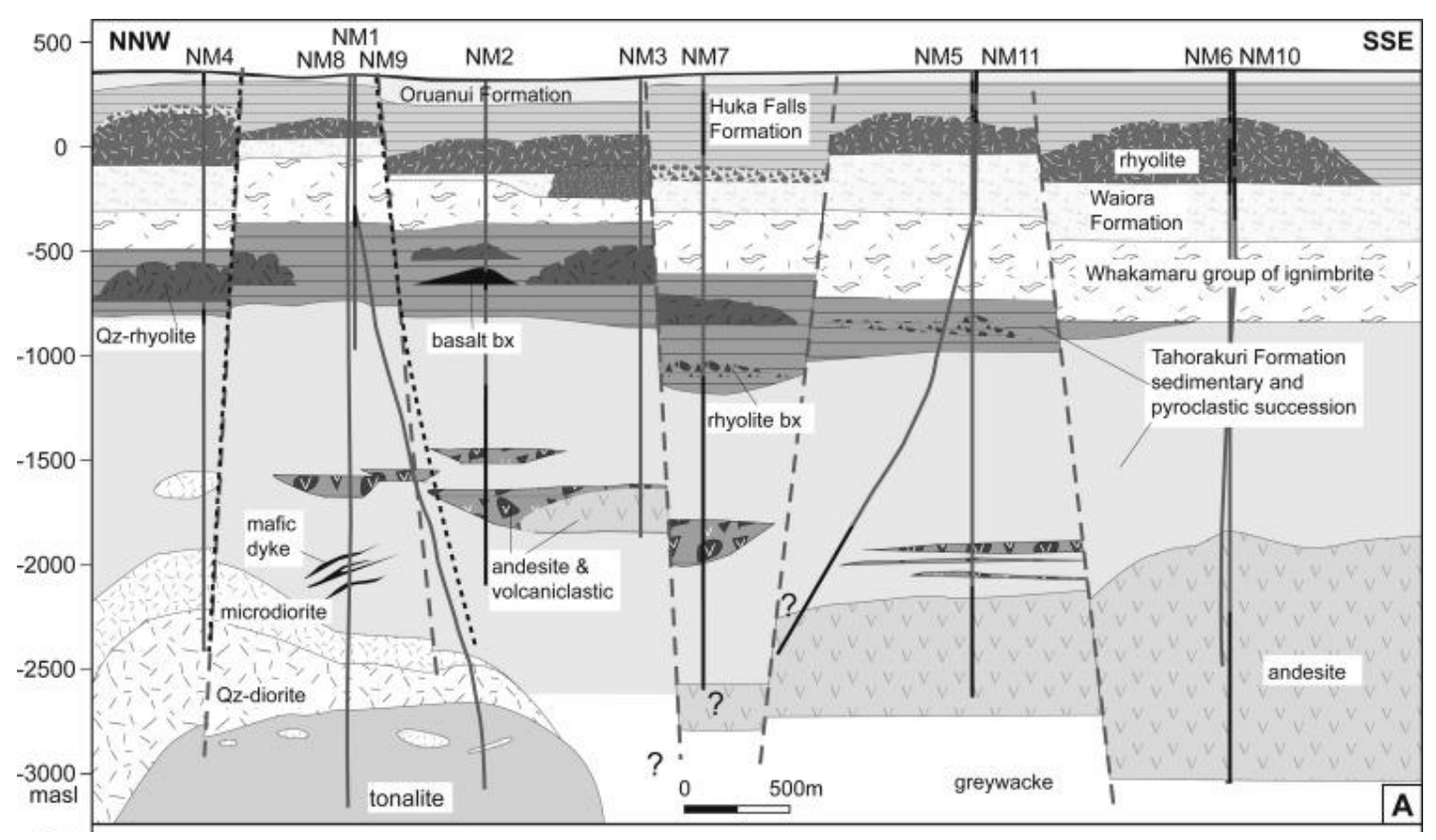

Figure 1.5: Cross-section of the Ngatamariki geothermal field depicting geothermal wells and general lithology (see Figure 1.4 for location) (Chambefort et al., 2016). Descriptions of the geologic units can be found in Section 1.2.2.

the current geothermal system (Arehart et al., 2002, Bertrand et al., 2015, Boseley et al., 2010).

Although the intrusive complex may have been presumed as the source of geothermal heat at Ngatamariki, the lack of alteration by and age of the aforementioned intrusive complex implied that this was not the source. A recent 3-D magnetotelluric survey of the area by Bertrand et al. (2015) illuminated a large conductive body beneath the Whakapapataringa Rhyolite Dome to the west of the field (Figure 1.3), and centered at approximately $5 \mathrm{~km}$ depth (Figure 1.6) (Bertrand et al., 2015). Bertrand et al. (2015) interpret this conductive zone to be a crystallizing magma body causing convection of high-temperature magmatic fluid and attribute it as the source of current geothermal energy at Ngatamariki.

The hydrology at Ngatamariki can be interpreted with reference to three aquifers. According to Boseley et al. (2010), these are a high-temperature $\left(260-300^{\circ} \mathrm{C}\right)$, high-permeability deep aquifer, an intermediate aquifer at $160^{\circ} \mathrm{C}$, and a shallow $15^{\circ} \mathrm{C}$ aquifer (Figure 1.7). These three aquifers are separated by smectite-rich clay layers, which are confirmed by resistivity data (Bertrand et al., 2015, Boseley et al., 2010, Chambefort et al., 2016). Isotherms (Figure 1.7) indicate a significant 


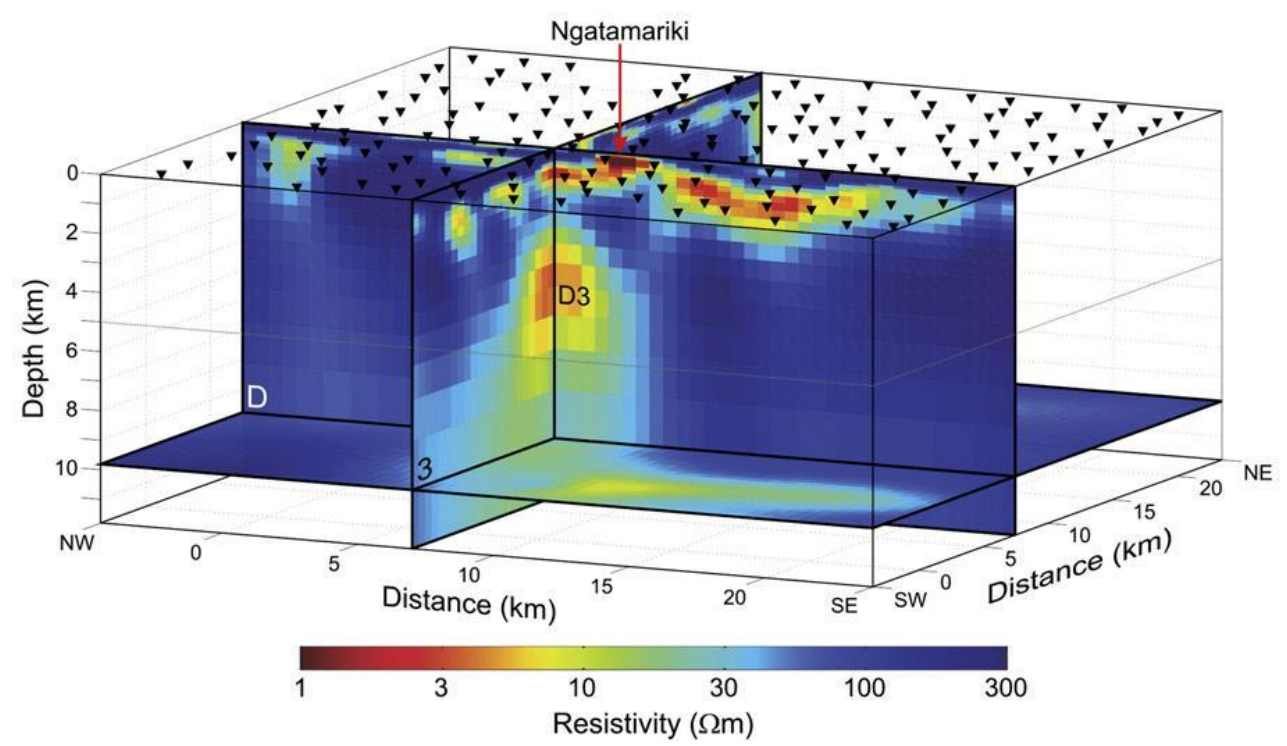

FiguRE 1.6: Orthogonal cross sections showing electrical resistivity beneath the Ngatamariki geothermal field (see Figure 1.3 for location) (Bertrand et al., 2015). Bertrand et al. (2015) name the deeper conductive body (labeled D3) as the source of the geothermal resource beneath Ngatamariki.

release of fluids from the high-temperature reservoir to the intermediate aquifer in the central field. Injection wells are needed at Ngatamariki to prevent downflow of fluid from the intermediate aquifer and cooling of the reservoir by pressure draw-down from production (Chambefort et al., 2016, Clearwater et al., 2015). 


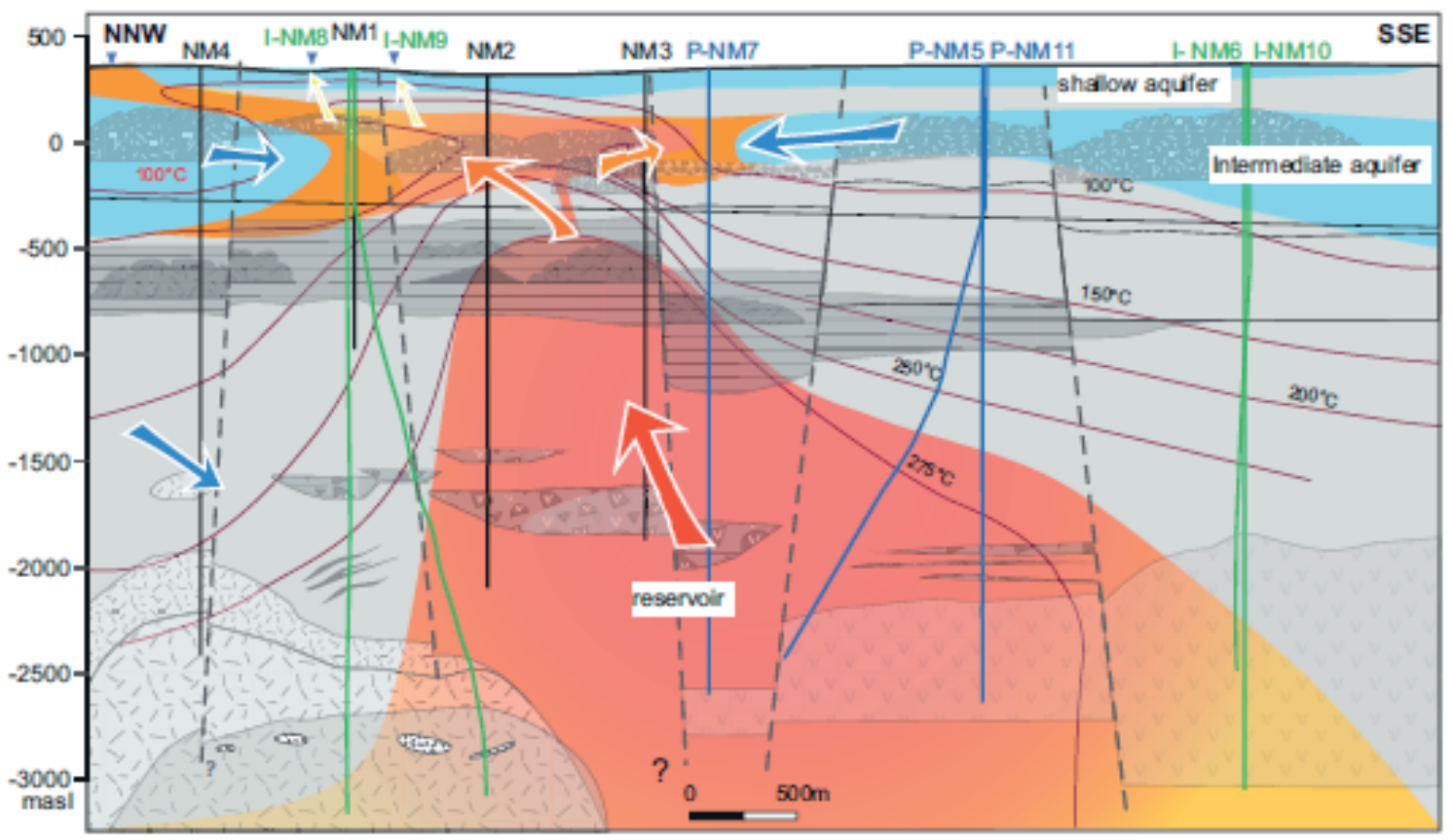

Figure 1.7: Cross-section of the Ngatamariki geothermal field depicting geothermal wells and hydrologic structure see Figure 1.4 for location) (Chambefort et al., 2016). There is a prominent leak at the central area of the field between the geothermal reservoir and the intermediate aquifer. 


\section{Chapter 2}

\section{Background}

\subsection{Geothermal fields and stimulation}

Temperature increases with depth below Earth's surface. This phenomenon is represented as the geothermal gradient, or the change in temperature over depth. On average, the geothermal gradient is $30^{\circ} \mathrm{C} / \mathrm{km}$, yet it can reach levels over $100^{\circ} \mathrm{C} / \mathrm{km}$ (Grant and Bixley, 2015). A high geothermal gradient is often caused by shallow magma bodies (Barbier, 2002). Heat may be transferred from these heat sources into adjacent pressurized fluids, or into a geothermal reservoir. By drilling into the geothermal reservoir, humans extract this heat and use it to produce power, termed 'geothermal energy production' (Barbier, 2002).

Geothermal fields can be divided into two categories based on the type of hydrothermal fluid in the reservoir: water-dominated or vapour-dominated (Barbier, 2002). Vapour-dominated fields contain dry steam at high pressures. A cap rock is necessary in these fields to keep steam from escaping at the surface (Barbier, 2002). Although vapour-dominated fields are not as common as water-dominated fields, they can generate significant amounts of energy (Barbier, 2002, Grant and Bixley, 2015). Water-dominated fields can be subdivided into hot water fields (containing water up to $100^{\circ} \mathrm{C}$ ) or wet steam fields (containing pressurized water above $100^{\circ} \mathrm{C}$ ) (Barbier, 2002, Grant and Bixley, 2015). Ngatamariki is a wet steam field, a high-temperature, fluid-dominated geothermal resource (Chambefort et al., 2014). The most commonly exploited geothermal fields contain wet steam. 


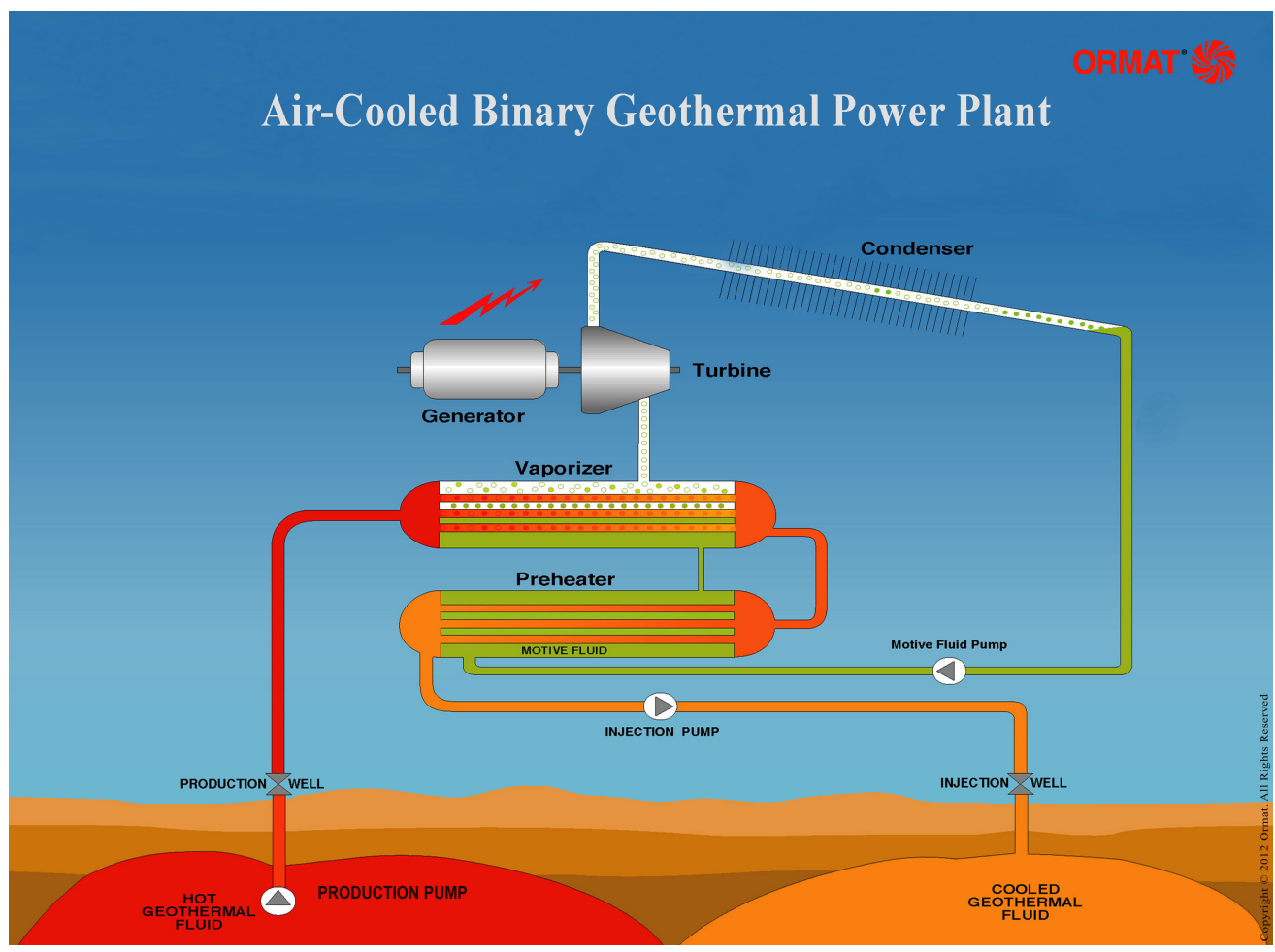

Figure 2.1: Diagram of a geothermal binary power plant with Ormat technology [http://www.ormat.com/solutions/Geothermal_Binary_Plant, 2016]. The power plant at Ngatamariki comprise a single binary unit able to produce $82 \mathrm{MW}$ of power (Clearwater et al., 2015).

Extracting heat from a reservoir generally requires the installation of production and injection wells by rotary drilling to penetrate the deep aquifer (Figure 2.1) (Barbier, 2002, Grant and Bixley, 2015). Production wells draw heated, pressurized fluid from the reservoir and injection wells pump cooled water or brine back into the reservoir (Barbier, 2002, Clearwater et al., 2015). In the case of Ngatamariki, a binary plant, fluid is drawn up through the production well and is used to heat and vaporize a secondary, more volatile fluid. The vapours from the secondary fluid drive a turbine, are condensed and are pumped back into a heating chamber (Figure 2.1). The cooled geothermal field is injected back into the reservoir (Grant and Bixley, 2015). Injection is used to aid in production and keep the geothermal resource viable by maintaining fluid pressures and aquifer volume (Clearwater et al., 2015).

It is standard practice is to inject cold water into the reservoir before geothermal production begins to improve the performance of geothermal injection wells (Clearwater et al., 2015, Grant et al., 2013). This injection improves reservoir permeability by cooling the rock mass adjacent to the well, thereby expanding 
fractures in the reservoir host rock (Grant et al., 2013). This process is known as "stimulating" the reservoir (Clearwater et al., 2015). This differs from hydraulic fracturing in which fractures are created and expanded by using significantly higher pressure during injection. To quantify how well an injection well performs, we use a quantity termed the Injectivity Index, or just injectivity, II. Formally, II is defined using the injection flow rate $W$ and the well head pressure, WHP (Clearwater et al., 2015).

$$
\mathrm{WHP}=P_{r}-\rho g Z+\frac{W}{\mathrm{II}}+K W^{2}
$$

where $P_{r}$ is reservoir pressure, $\rho$ is density, $g$ is acceleration due to gravity, $Z$ is downhole depth, $W$ is mass flow rate, and $K$ is a coefficient that describes frictional forces (Grant et al., 2013). As the injection continues over time, II will increase as a power of time $t$ (Figure 2.2) (Clearwater et al., 2015, Grant et al., 2013). II is measured in (t/hr/bar). That is,

$$
\mathrm{II}=C t^{n} \text {, such that } C \in \mathbb{R}, n \in[0.4,0.7]
$$

Clearwater et al. (2015) shows empirically that $n=0.7$ for Ngatamariki (Figure 2.2). There are areas of the stimulation curve, where the trend of II does not agree with a slope of 0.7 . This is ascribed to transient phases in stimulation and not true stimulation of the reservoir (Clearwater et al., 2015). Most importantly, the permeability in fractured, hydrothermal rock increases when a front of colder temperature is applied via injection of cold water (Clearwater et al., 2015, Grant et al., 2013). This increase is caused by thermal contraction of reservoir rock, which can be more significant than fluid pressure at driving fractures open (Grant et al., 2013).

\subsection{Exploration and production at Ngatamariki}

Geophysical study of the TVZ began in the 1960s with an electrical resistivity survey spanning the entire zone with the intention of resource identification (Boseley et al., 2010). An observed low-conductivity anomaly indicated the need for further investigation at Ngatamariki (Boseley et al., 2010, Chambefort et al., 2016, Clearwater et al., 2015). 


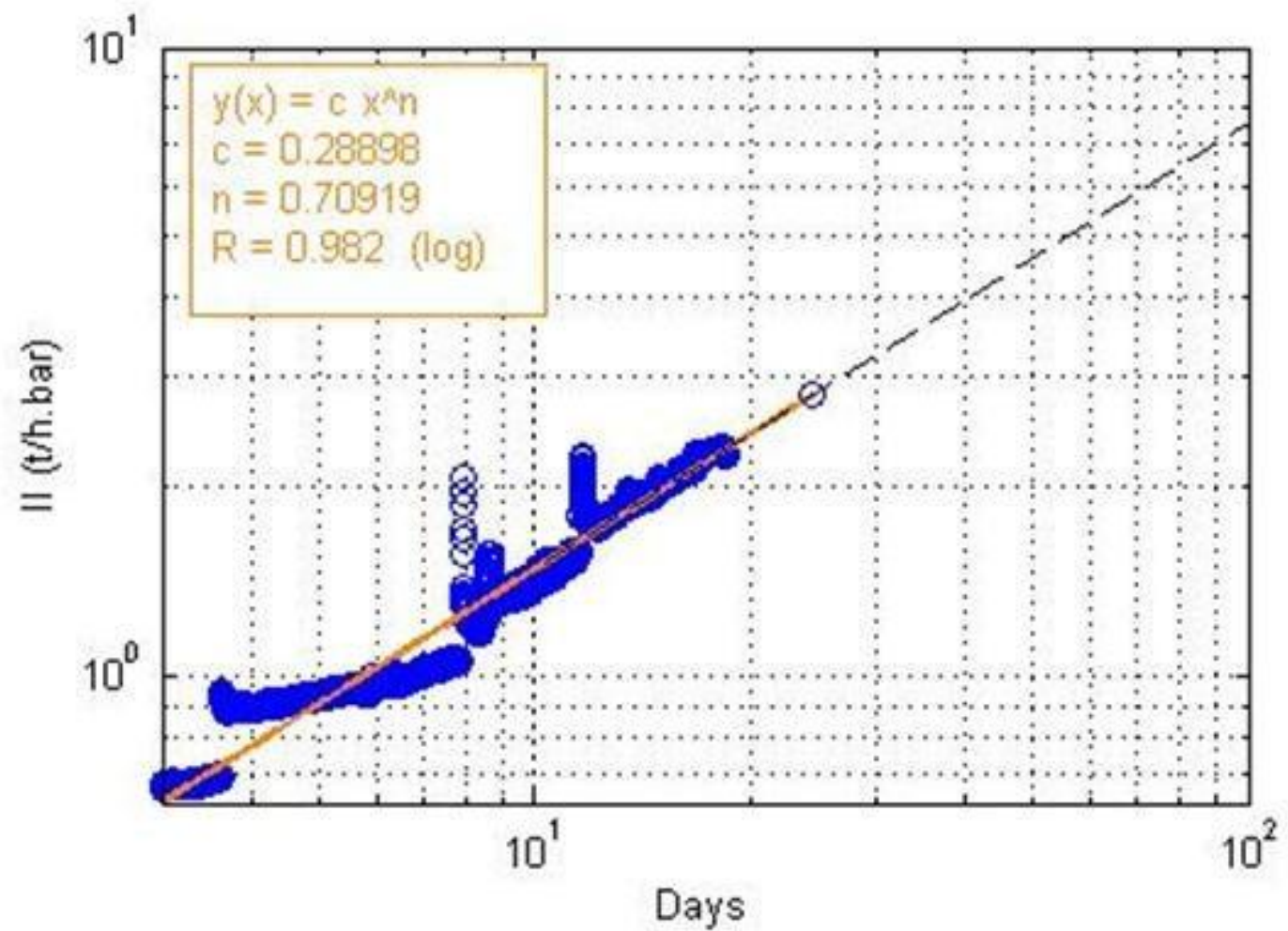

FIgURE 2.2: Log-log scatter plot of NM8 Injectivity Index during the 31 day stimulation period (Clearwater et al., 2015). The plot shows that injectivity is proportional to nth power of time, $\mathrm{II}=t^{n}$ or in $\log -\log$ space, $\log (\mathrm{II})=n \log (t)$.

Exploration at the Ngatamariki geothermal field began in 1980 with the drilling of four wells (NM1-NM4) using rotary drilling and abundant coring (Figure 2.3) (Chambefort et al., 2016). Wells NM2 and NM3 encountered a high permeability geothermal resource at temperatures above $280^{\circ} \mathrm{C}$ (Figures 1.4 and 1.7) (Boseley et al., 2010, Chambefort et al., 2016, Clearwater et al., 2015). In 2004, Mighty River Power (MRP) gained access to the field along with Tauhara Development Limited and Rotokawa Joint Venture Limited and drilled three more wells (NM5NM7) in order to delineate the resource. From 2008 to 2010, multiple conceptual models were developed in order to build constraints on the resource extent (Boseley et al., 2010, Clearwater et al., 2011, Dempsey et al., 2013). Additionally, in 2010 MRP and Tauhara North No. 2 Trust were granted resource consent to withdraw 60,000 tonnes/day of geothermal fluid (Chambefort et al., 2016, Clearwater et al., 2015). In the same year, Boseley et al. (2010) performed a smaller scale magnetotelluric, survey which revealed a 7 to $12 \mathrm{sq}$. km conductive anomaly.

In 2011, MRP commenced construction of the $82 \mathrm{MW}$ plant and began rotary drilling of four more wells (NM8-NM11) (Chambefort et al., 2016, Clearwater 


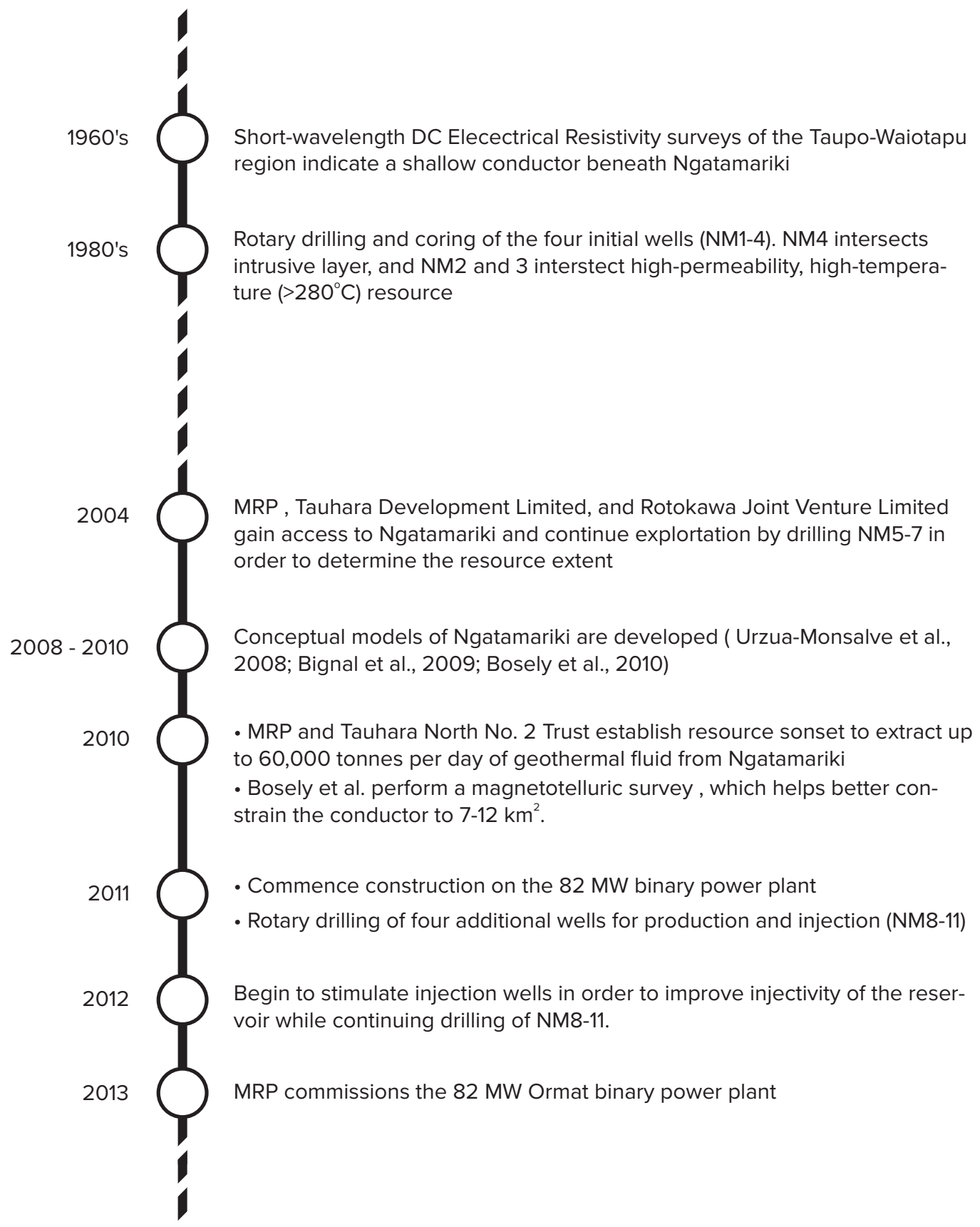

Figure 2.3: Timeline for development at the Ngatamariki geothermal field.

et al., 2015). Starting in June 2012 with the completion of the first injection well, NM8, MRP started stimulation tests to asses and improve injectivity of the reservoir (Clearwater et al., 2015, Dempsey et al., 2013). NM9 and NM10 were subsequently stimulated via injection of cold river water. In 2013, the plant was commissioned and production commenced (Clearwater et al., 2015). 


\subsubsection{Injection well NM8}

NM8 sits at the northern end of the Ngatamariki geothermal field and reaches $3600 \mathrm{~m}$ depth (Figure 1.5) (Chambefort et al., 2016). It was constructed as an injection well and completed in May 2012. The formations that NM8 intersects are detailed in Section 1.2.2. NM8 underwent stimulation from June 7 through July 8 , over which the Injectivity Index improved from 0.4 to $2.7 \mathrm{t} / \mathrm{hr} / \mathrm{bar}$, following a stimulation slope of $n=0.7$ (Section 2.1) (Clearwater et al., 2015). Although permeability at the base of the well is low due to its position within the intrusive pluton, feed zones higher along the borehole allow for stimulation to be effective (Clearwater et al., 2015). A feed zone is an area that allows for exchange between the fluid within the well and the fluid in the reservoir. When injecting cold fluids, this allows fluid release into the reservoir before reaching the bottom of the well (Glynn-Morris et al., 2011).

\subsection{Overview of induced seismicity sources}

Induced seismicity is the process by which human activity causes significant enough perturbations in the stress field to cause a fault to slip, or a fracture to open, resulting in an induced earthquake. Humans may induce earthquakes in myriad ways including mining, building reservoirs, and injecting fluid below the surface. Here we present an overview of the mechanisms of induced seismicty and the human activities that contribute to causing seismicity.

\subsubsection{Mechanisms of induced seismicity}

An earthquake is a release of energy at the time slip occurs along a failure plane. Failure occurs when the shear stress $\tau_{s}$ exceeds a scalar multiple of the normal stress $\sigma_{n}$ and the cohesion of the parent rock $C_{f}$ (Mossop, 2001, Shapiro, 2015).

$$
\tau_{s} \geq \mu_{s} \sigma_{n}+C_{f}
$$

The equation for $\tau_{s}$ is called the Coulomb failure criteria, and if a fault were to fail due to these conditions, we call this Coulomb failure. Under a given stress regime, a fault plane may fail if it is in a favourable orientation, otherwise it will 
not fail at all. Reducing normal stress by subsurface injection of cold fluid may cause a fault to fail. We consider two possibilities by which this is achieved: (1) a reduction for effective normal stress due to the increase of pore fluid and (2) thermal contraction due to temperature differences between the injected fluid and the reservoir (Bodvarsson, 1972, Mossop, 2001, Shapiro, 2015).

Addition of fluids into a system, reduces $\sigma_{n}$, so that for a pore fluid pressure of $p_{f}$, the effective normal stress becomes $\sigma_{\text {eff }}=\sigma_{n}-p_{f}$ (Shapiro et al., 2003, Shapiro, 2015). Injected fluid causes diffusion of pore fluid pressure from the well into the surrounding reservoir (Shapiro, 2015). Being diffusive, the radial extent $(R)$ of a pore fluid pressure front in an isotropic medium, a time $t$ after injection at a point can be described as $R=\sqrt{4 \pi D t}$, where $D$ is the hydraulic diffusivity of the reservoir (Shapiro, 2015). Hydraulic diffusivity is a measure of how easily a fluid can move through a medium, and is measured in $\mathrm{m}^{2} / \mathrm{s}$. Once injection starts, it is common to see microseismicity induced between the source of fluid and the radial front of pore fluid pressure (Figure 2.4) (Shapiro et al., 2003, Shapiro, 2015). Shapiro (2015) provides a overview of many case studies for which microseismicity is induced by an increase in pore fluid pressure, via poroelastic stresses. We can estimate hydraulic diffusivity based on the envelope method illustrated in Figure 2.4. This method of diffusivity determination is only an estimate because in reality, hydraulic diffusivity is represented by a matrix, and will only be represented by a scalar if the reservoir is isotropic with respect to fluid flow. For this reason, we do not require $100 \%$ of the events to fall within the front of pore fluid pressure (Figure 2.4), as they could potentially be occurring in a fast-direction of fluid flow (Shapiro and Dinske, 2009, Shapiro et al., 2003, Shapiro, 2015).

Pore fluid pressure increase can cause earthquakes regardless of the temperature of the reservoir. However, at Ngatamariki the temperature difference between injected fluid and the reservoir provides another mechanism of induced stress perturbation (Bodvarsson, 1972, Clearwater et al., 2015, Grant et al., 2013, Sewell et al., 2015, Sherburn et al., 2015b). When a cool injectate is introduced into a hot geothermal reservoir, temperature advects and carries heat to the reservoir and host rock (Bodvarsson, 1972, Mossop, 2001). As the reservoir cools, pre-existing fractures contract, reducing the normal stress by increasing the volume of the cracks and hence decreasing density. Thermal contraction has been shown to be significant enough to cause seismicity (Bodvarsson, 1972, De Simone et al., 2013, Sewell et al., 2015, Sherburn et al., 2015b). Sherburn et al. (2015b) and Sewell et al. 


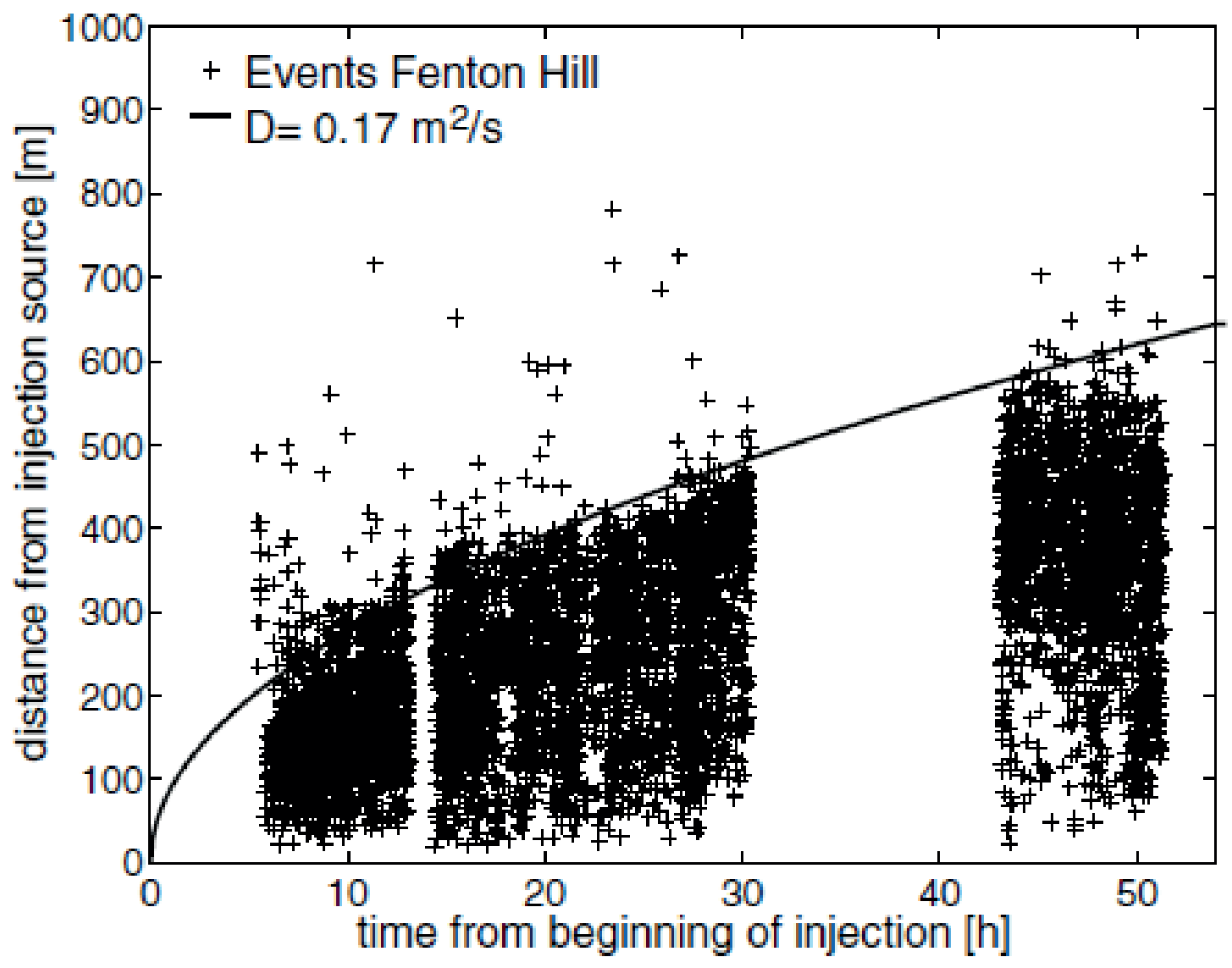

FiguRE 2.4: Example of a diffusion envelope for microseismicity during hydraulic fracturing experiments at Fenton Hill, New Mexico. Events are plotted by when they occurred from the start of injection versus how far from injection they occurred. The curve represents the extent of a fluid pressure front extending radially from the point of injection assuming a hydraulic diffusivity, D, of $0.17 \mathrm{~m}^{2} / \mathrm{s}$ (Shapiro et al., 2003)

(2015) attribute induced microseismicty at the Rotokawa geothermal field, adjoining Ngatamariki to the south, to be caused primarily from thermal contraction as opposed to pore fluid pressure (Figure 1.3, labeled "RK"). During June-July stimulation testing at NM8, injected fluid was approximately $20^{\circ} \mathrm{C}$, whereas the reservoir is approximately $300^{\circ} \mathrm{C}$ (Clearwater et al., 2015, Dempsey et al., 2014). This is enough temperature difference to induce seismicity via thermal contraction (Sewell et al., 2015, Sherburn et al., 2015b).

\subsubsection{Locations of induced seismicity}

Here we consider the variety of anthropogenic sources for induced seismicity including reservoirs, mining, and injection. 
Reservoirs: Studies of induced seismicity started in the 1930s with the reporting of increased seismicity following construction of water reservoirs. Until recently, the largest earthquake induced by reservoir loading was in Koyna, India, with a 6.5 $M_{w}$ event (Gupta and Rastogi, 1976). In 2008, the $7.9 M_{w}$ Wenchuan earthquake surpassed the Koyna event as the largest recorded reservoir induced event (Kerr and Stone, 2009). Reservoirs induce seismicity by increasing the pore fluid pressure at depth (Gupta and Rastogi, 1976, Kerr and Stone, 2009).

Mining: Mining offers several mechanisms for inducing seismicity, including changing the overburden stress by removing material, explosive blasts, and mine collapse. For example, in the 1970s Witwatersrand gold fields in South Africa were being excavated by removal of tabular sections of rock (Cook, 1976). Stress changes resulted from the collapse of voids left by mining, causing a high rate of seismicity within a few hundred meters of the mine (Cook, 1976). The largest known mining induced event is a magnitude $5.4 M_{w}$ event in 1989 induced by rockbursts in Volkerhausen, Germany (Bennett et al., 1994, Knoll, 1990). Bennett et al. (1994) show that the Volkershausen events had implosive components in their sources, implying an induced mechanism.

Injection: Healy et al. (1968) describes a correlation between earthquakes occuring in 1968 near Denver, Colorado and the injection of wastewater. By 1976, the U.S. Geological Survey performed an experiment at the Rangley Oil Field, Colorado to gain insights into the induced seismicity that Healy et al. introduced (Raleigh et al., 1976). Raleigh et al. (1976) confirmed the Healy et al. (1968) hypothesis that earthquakes can be triggered by increased pore fluid pressure. Injection has induced earthquakes in boreholes as deep as $9.1 \mathrm{~km}$ during Germany Continental Deep Drilling Program (Harjes, 1997). Earthquakes were induced just above the base of the injection well and closely followed typical behaviour of seismicity induced by pore fluid pressure (Harjes, 1997, Shapiro, 2015).

The TVZ: Several studies have analysed induced seismicity related to geothermal production and injection. Although little work has been published on induced microseismicity at Ngatamariki, there have been several studies across the TVZ and at Ngatamariki's neighbouring field, Rotokawa (Figure 1.3) (Sewell et al., 2015, Sherburn et al., 2015b).

Large-scale geothermal energy production in the TVZ began in the 1950s, but injection did not commence until the 1980s at Wairakei (Sherburn et al., 2015a). 
Injection here has dual purposes, namely the disposal of geothermal brine and to maintain the viability of the geothermal resource. Most modern fields rely on deep injection, far below the depth that production wells draw fluid from. This eliminates interaction with intermediate and shallow aquifers which may cause fluid discharge at the surface (Sherburn et al., 2015a).

Sherburn et al. (2015b) and Sewell et al. (2015) present a detailed study of microseismicity at the Rotokawa geothermal field from 2008 to 2012 . They show that there is a significant spatiotemporal relationship between injection/production and the occurrence of microseismicity. Using the microseismicity, they mapped and characterized the Central Field Fault, a feature that acts as a conduit along its strike, but a as a barrier to fluid moving normal to strike (Sewell et al., 2015, Sherburn et al., 2015b). Additionally, due to changing of injection and production, Sherburn et al. (2015b) and Sewell et al. (2015) observe that the locations of seismicity changed with differing injection well sites. Lastly, Sherburn et al. (2015b) and Sewell et al. (2015) attribute the mechanism of seismicity to thermal stresses caused by cold fluid injected into the hot reservoir rock (Section 2.3.1).

\subsubsection{Detection of induced seismicity}

In order to study the possible effects of anthropogenic factors on seismicity, we need to have a catalogue of earthquakes to consider. Historically, earthquake signals are detected within a background signal without knowing the source parameters or the waveform of the event (Gibbons and Ringdal, 2006). To detect an earthquake within a background signal, one would compare the power of the time series over a short time interval (STA) with the power of the time series over a long time interval (LTA). The ratio is known as the short term-long term average (STA/LTA), and described in terms of the signal-to-noise ratio (SNR) (Freiberger, 1962). When the power in the short term is subsequently large relative to the power over the long interval, or the SNR is large enough to surpass a threshold, then there is a detection (Figure 2.5). The STA/LTA method has been used extensively since it was introduced by Freiberger (1962). This is a suitable method for detecting strong signals, but once the signal-to-noise ratio (SNR) becomes too weak, the STA/LTA method is no longer effective (Gibbons and Ringdal, 2006). The STA/LTA method will no longer be able to reliably distinguish a signal from noise. 


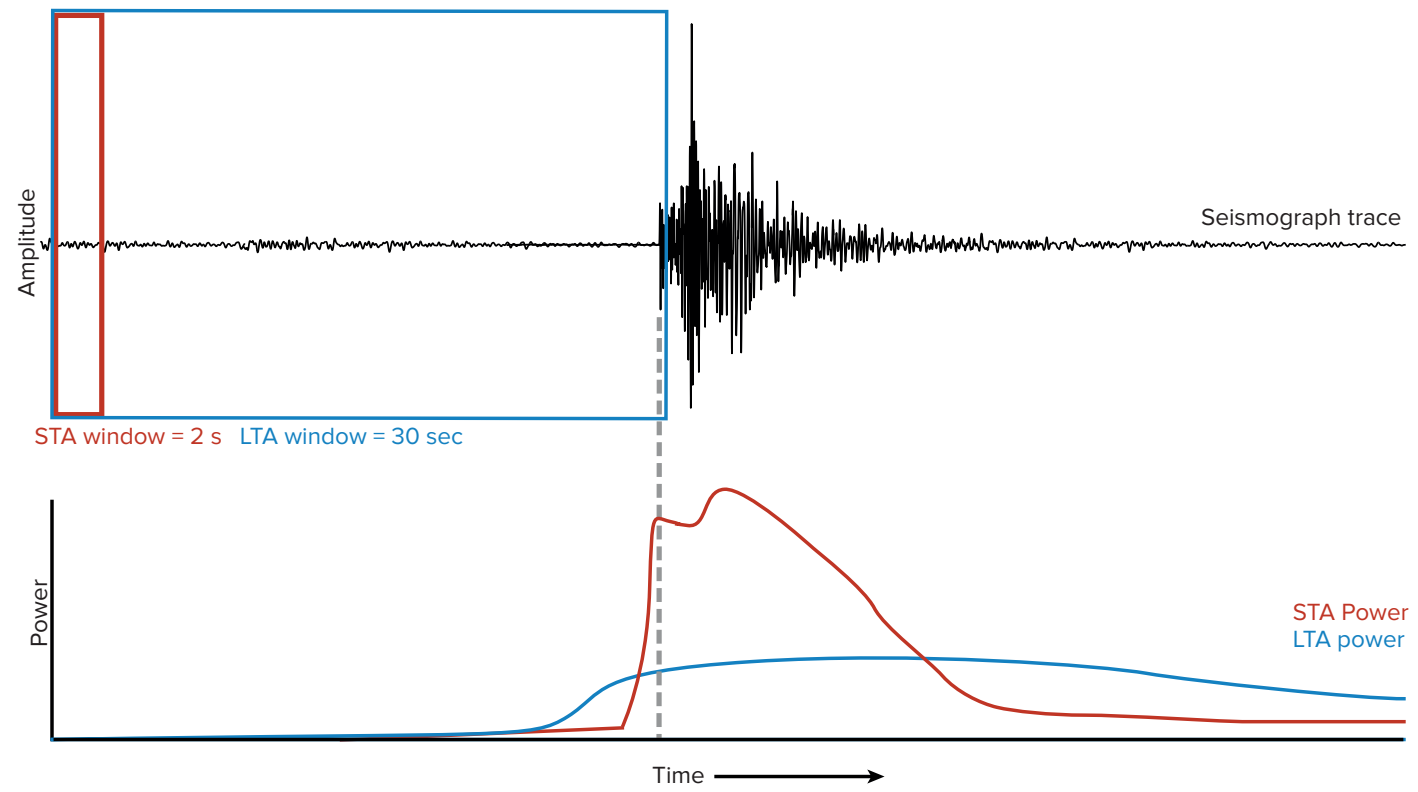

FIgURE 2.5: STA/LTA methodology schematic. An example of a signal is plotted above with examples of a short-term and long-term window. Power of the signal is calculated for each short-term and long-term window, which is displayed below. An event is said to occur when the extrema in the STA is large enough relative to the LTA, the timing of which is illustrated by the dashed line.

Geothermal areas are known to have high levels of seismic noise (Clacy, 1968, Douze and Sorrells, 1972, Goforth et al., 1972). We are interested in small magnitude earthquakes $\left(<3 \mathrm{M}_{\mathrm{L}}\right)$, which have low SNR in a highly noisy geothermal area. One solution for detecting small events in noisy signals is to use waveform cross-correlation of the background signal with a known, filtered earthquake signal, or template (Anstey, 1964, Chamberlain et al., 2014, Gibbons and Ringdal, 2006, Schaff and Waldhauser, 2010, Shelly et al., 2007, Van Trees Jr et al., 1968). As will be described in Chapter 3, cross-correlation quantifies the similarity between two signals. Cross-correlating the template with the background signal will quantify if at any point in time the background signal displays similarity to the template. This is known as a matched-filter methodology (Figure 2.6) (Anstey, 1964, Shelly et al., 2007, Van Trees Jr et al., 1968). Matched-filtering is widely used in the larger scientific/mathematic/engineering fields; however, widespread use in seismology is more modern (Brown et al., 2008, Chamberlain et al., 2014, Gibbons and Ringdal, 2006, Schaff and Waldhauser, 2010, Shelly et al., 2007).

Several studies have used matched-filtering to detect low-frequency earthquakes on local networks in Japan, the Alpine Fault, and Alaska (Brown et al., 2008, Chamberlain et al., 2014, Shelly et al., 2007). Here, we use methods developed by 

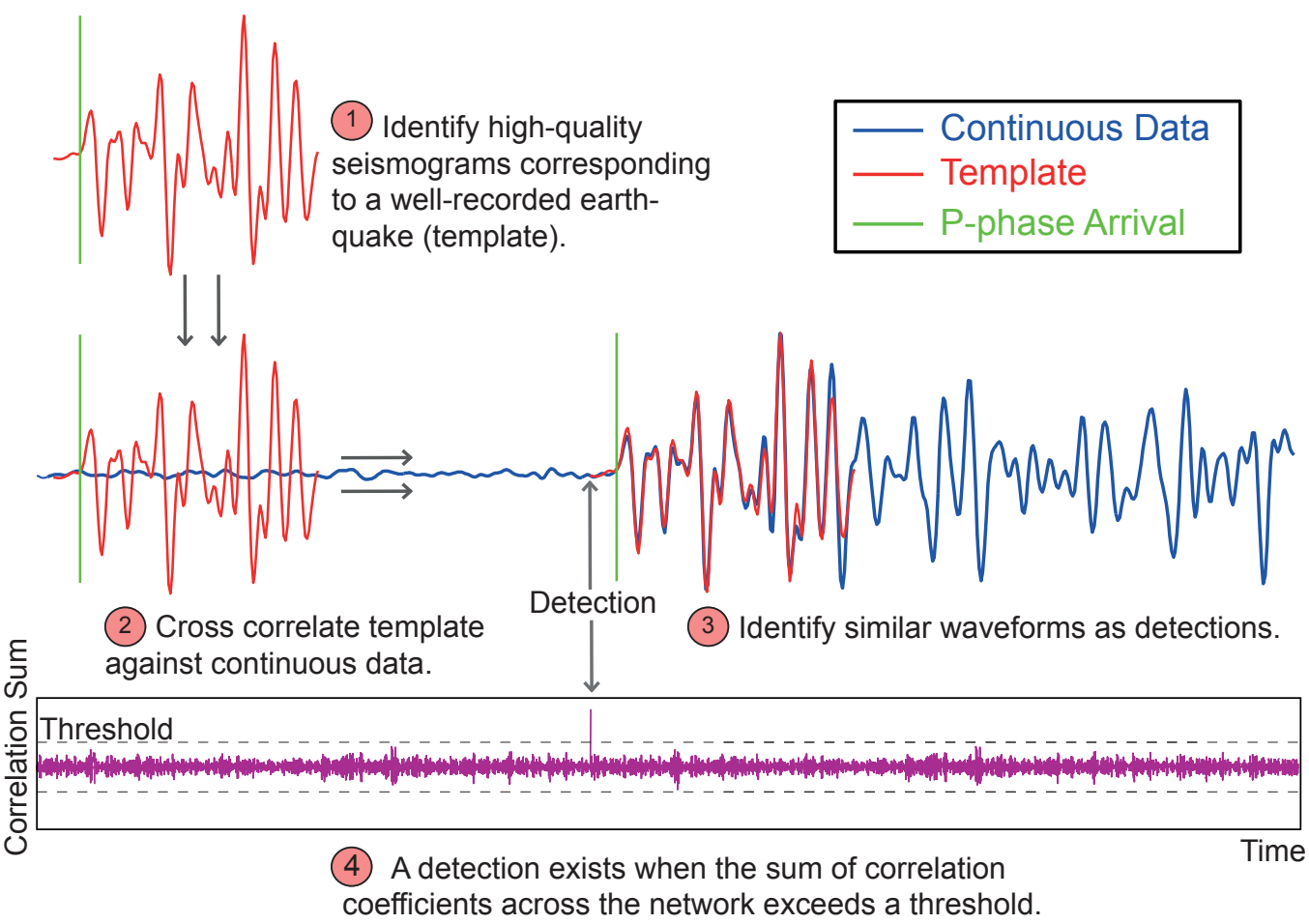

FiguRE 2.6: Matched-filter methodology schematic.

Shelly et al. (2007) and Chamberlain et al. (2014) which implement the matchedfilter routine using Matlab. Low-frequency earthquakes differ from microseismicity, but the matched-filter method can be applied to either. Geothermal fields are also a suitable place to use matched-filtering due to the low SNR of most seismic signals of interest. Rowe et al. (2002a) adapts cross-correlation to make precise phase arrival picks for earthquakes in the Soultz geothermal reservoir. Rawlinson (2011) applies the matched-filter methodology (based on the method developed by Rowe et al. (2002b)) to the Rotokawa geothermal field (Figure 1.3). Our study aims to continue the process of adapting matched-filter methods for use in the TVZ. 


\section{Chapter 3}

\section{Data and Methodology}

In Chapter 3, we describe the data available and the methodology we use for analysis. We use two types of data: (1) seismic waveform data and (2) geothermal field data. Seismic data consists of day long seismographs and timing of known events, whereas geothermal field data consists of locations and amounts of fluid release into the subsurface.

The methodology description begins with an precise definition of cross-correlation, followed by a flow of the matched-filter method. We give a detailed explanation of the methods presuming the reader has a university level of mathematical knowledge. For further explanation of concepts upon which we build the methods, see Lyons (2010).

\subsection{Data}

\subsubsection{Seismic data}

Mighty River Power (MRP) began operation of the Ngatamariki seismic network in 2012 just before stimulation of NM8 (Figure 3.1). At the time, the network consisted of seven stations (labeled 'NS', Figure 3.1). MRP also operates the Rotokawa network immediately south of Ngatamariki (labeled 'RT', Figure 3.1) (Sherburn and Bourguignon, 2013). Instruments in the Rotokawa and Ngatamariki networks are Geospace GS-11D 4.5 Hz seismometers with a $200 \mathrm{~Hz}$ sample rate (Rawlinson, 2011). GS-11D instruments have highest response at frequencies 
between 4.5 to $100 \mathrm{~Hz}$ (Table 3.1). Since installation in 2012, the Ngatamariki and Rotokawa networks have been operated independently, despite their close proximity (Figure 3.1) (Sherburn and Bourguignon, 2013). MRP supplied continuous seismic data from the ten stations that were operating in June and July 2012 for this project (Steven Sewell, MRP, pers. comm., 2015). These data are in compact miniSEED format and segmented into day-long intervals (00:00 to 23:59) by station and component.

We use additional data from the New Zealand National Seismograph Network station Whakapapataringa, or WPRZ (Figure 3.1) (Petersen et al., 2011). Waveform data from all stations in the national network are available through GeoNet (www.geonet.org.nz). Station WPRZ began operation in March 2010 and comprises a Lennartz LE-3DliteMkII short-period instrument with a sampling rate of $100 \mathrm{~Hz}$ (www.lennartz-electronic.de, 2015). The Lennartz instrument is lowpower and low-noise; it has the highest response at frequencies from 1 to $100 \mathrm{~Hz}$ (www.lennartz-electronic.de, 2015).

TABLE 3.1: Seismic stations around Ngatamariki

\begin{tabular}{ccccc}
\hline Station & Latitude & Longitude & Elevation $(\mathrm{m})$ & Instrument \\
\hline NS02 & -38.5543 & 176.1623 & 375 & Geospace GS-11D \\
NS03 & -38.5233 & 176.2092 & 327 & Geospace GS-11D \\
NS04 & -38.5425 & 176.2257 & 345 & Geospace GS-11D \\
NS06 & -38.5655 & 176.1945 & 407 & Geospace GS-11D \\
NS07 & -38.5608 & 176.2270 & 345 & Geospace GS-11D \\
NS08 & -38.5303 & 176.1855 & 345 & Geospace GS-11D \\
NS09 & -38.5755 & 176.1628 & 451 & Geospace GS-11D \\
RT02 & -38.6017 & 176.1765 & 346 & Geospace GS-11D \\
RT07 & -38.6045 & 176.2065 & 320 & Geospace GS-11D \\
RT11 & -38.5993 & 176.1942 & 330 & Geospace GS-11D \\
WPRZ & -38.5197 & 176.1623 & 519 & Lennartz LE-3DliteMkII \\
\hline
\end{tabular}

In order to apply the matched-filter method, we require a set of known seismic events (Shelly et al., 2007). The Institute of Geological and Nuclear Sciences (GNS Science) carries out detection and location routines using the Mighty River Power's Ngatamariki and Rotokawa networks (Figure 3.1) (Sherburn and Bourguignon, 2013). Detection is performed automatically using an STA/LTA method (Sherburn and Bourguignon, 2013). Initial locations are also computed automatically. Events detected by STA/LTA with more than 10 phase arrivals (across components) undergo manual phase-pick refinement (Sherburn and Bourguignon, 


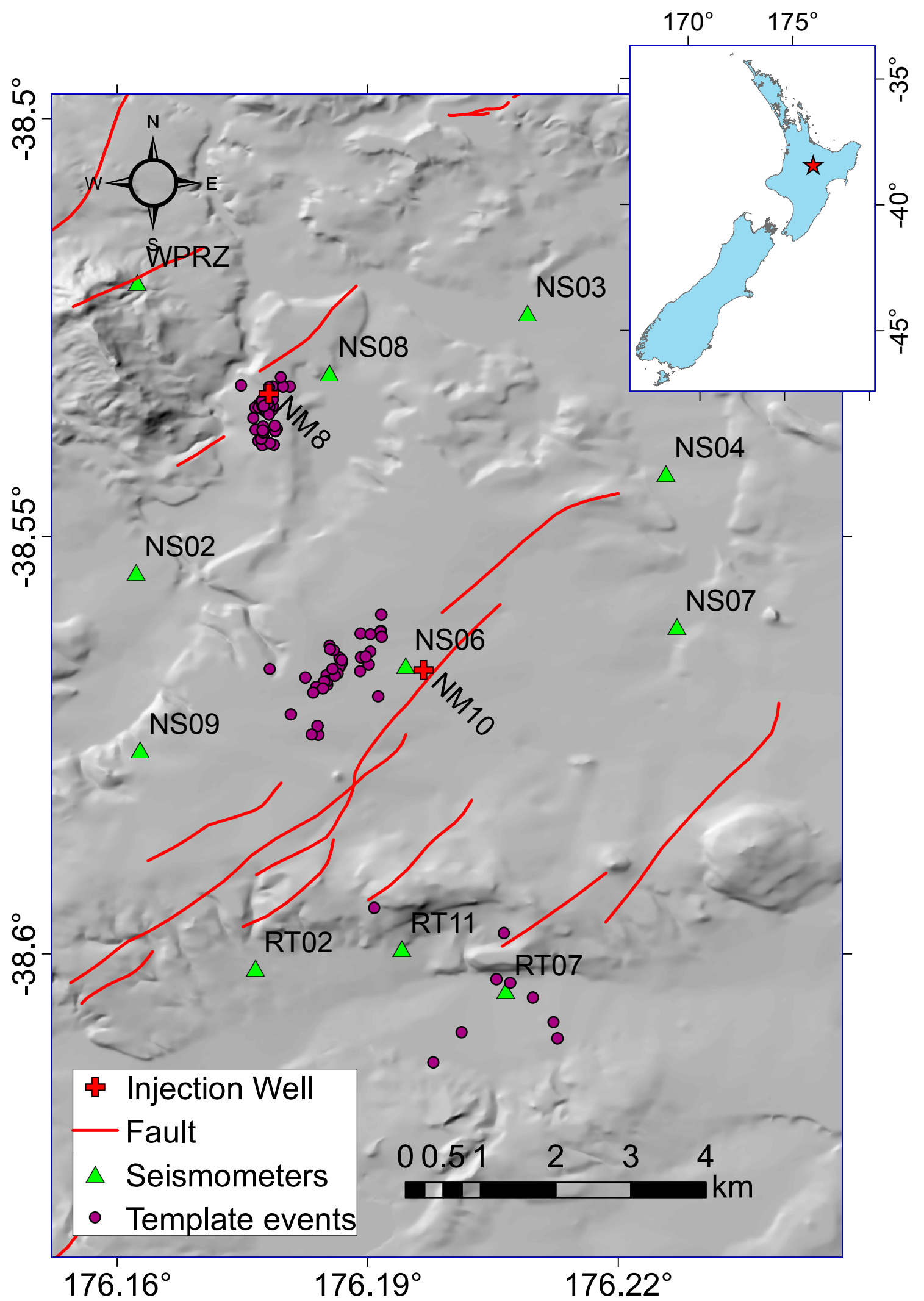

Figure 3.1: Map of the Ngatamariki geothermal field. Seismometers, two relevant injection wells (NM8 and NM10), and a priori known template events are shown.Faults were provided from (Langridge et al., 2016) 
2013). Next, in order to improve locations, GNS Science researchers perform waveform cross-correlation using BCSeis, a method developed by Du et al. (2004). This generates lag times between pairs of events that are subsequently used for double difference relocation (Du et al., 2004). The double-difference methodology determines more accurate relative locations for a set of closely clustered events (Waldhauser and Ellsworth, 2000). The known events, or templates are shown on Figure 3.1.

\subsubsection{Geothermal field data}

There are 11 wells at Ngatamariki which intercept the geothermal reservoir (Figure 1.4) (Chambefort et al., 2016). Four wells (NM6, NM8, NM9, NM10) are currently used for injection while three (NM5, NM7, NM11) are used for production (Boseley et al., 2010, Chambefort et al., 2016). Rotary drilled holes often deviate from vertical. Three of the wells at Ngatamariki (NM5, NM9, NM10) deviate significantly (Chambefort et al., 2016). It is important to know each well's orientation at depth when considering induced seismicity. A well is not always entirely impermeable along its length (Glynn-Morris et al., 2011). Knowledge of a feed zone location can be significant for inferring from where pore fluid pressure or temperature changes emanate. Mighty River Power has provided depth profiles and feed zone locations for all 11 wells at Ngatamariki, however we focus on NM8 and NM10 in this study. The principal feed zone for NM8 sits at 2000 meters below sea level and is approximately 20 meters long (Steven Sewell, MRP, pers. comm., 2015).

For the NM8 stimulation period (June - July 2012) MRP measured injection flow rate (tonnes/hour) and well head pressure, or WHP (bars). These are both measured every five minutes throughout June and July 2012 (Steven Sewell, MRP, pers. comm., 2015). We will compare these data with microseismicity rates in order to infer whether seismicity is induced through exploitation or naturally occurs.

Starting in May, MRP oversaw the drilling of injection well NM10, and in July the borehole reached the level of the reservoir (Steven Sewell, MRP, pers. comm., 2015). During drilling, operators pump fluid down into the borehole to aid in the drilling process. Fluid flows down the drilling pipe, out through the bit, then back up towards the surface. However, uncontrolled flow may leak into the surrounding 


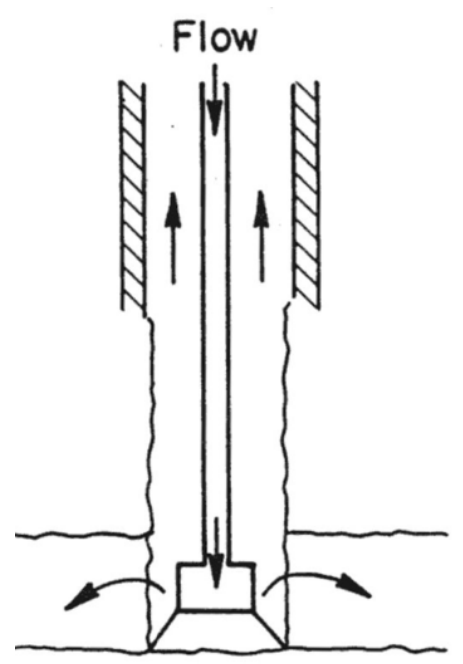

a. Partial loss

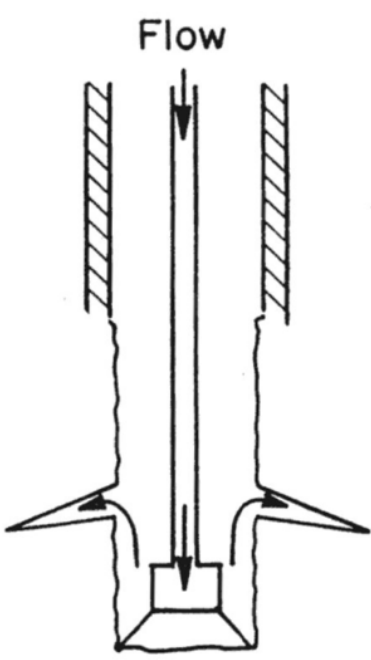

b. Total loss

Figure 3.2: Schematic of (a) partial and (b) total fluid loss during drilling [http://petrowiki.org/Lost_circulation, 2016]

formation instead of circulating back towards the surface. This situation is called partial, or total loss of circulation (Figure 3.2) (http://petrowiki.org/Lost_ circulation, 2016). In mid-late July, MRP experienced loss of drilling fluids into the formation (Steven Sewell, MRP, pers. comm., 2015). Rate and cumulative amounts of fluid loss were recorded during the occurrences. We will use these data to compare with microseismicity rates near NM10.

\subsection{Cross-correlation theory}

Matched-filtering is based upon cross-correlation of a known signal, or template, with the continuous background waveform, or image (Briechle and Hanebeck, 2001, Lewis, 1995). The following is a detailed, mathematical approach to crosscorrelating template $g$ with image $f$ at time $\tau$.

We define a time series $f(t)$ of length $M$ as:

$$
f(t)=t_{1}, t_{2}, t_{3}, \ldots, t_{M}, \text { such that } t_{i} \in \mathbb{R}, i=1,2,3, \ldots, M .
$$

We will now show how to quantify the similarity of $g(t)$ (template, of length $N$ ) and $f(t)$ (image, of length $M$ ), at time $\tau$. This could be thought of as how far apart $g$ and $f$ are from each other (i.e. Euclidean distance) (Lewis, 1995). The 
distance $d_{f, g(\tau)}$ is defined as:

$$
d_{f, g(\tau)}^{2}=\sum_{t}[f(t)-g(t-\tau)]^{2}
$$

where $f$ is the image, and the sum is over times $t$ under the interval containing the template $g$ at time $\tau$. By the binomial theorem,

$$
d_{f, g(\tau)}^{2}=\sum_{t}\left[f^{2}(t)-2 f(t) g(t-\tau)+g^{2}(t-\tau)\right]
$$

The term $\sum g^{2}(t-\tau)$ is constant, and if $\sum f^{2}(t)$ is also constant, then the remaining component of distance is:

$$
c(\tau)=\sum_{t} f(t) g(t-\tau)
$$

There are however several disadvantages to using $c(\tau)$ for matched-filtering (Lewis, 1995).

1. If $\sum f^{2}(t)$ (the image energy) is not constant, then calculating $c$ will be skewed . For example, the correlation between the template and an exact matching region in the image may be less than between the template and a region with a large spike in amplitude.

2. The range of $c(\tau)$ is dependent on the length of the template, $N$.

3. $c(\tau)$ varies with changes in image amplitude.

A better approach to calculating similarity is by calculating the correlation coefficient $\gamma$ (Briechle and Hanebeck, 2001, Lewis, 1995):

$$
\gamma(\tau)=\frac{\sum_{t}\left[f(t)-\bar{f}_{\tau}\right][g(t-\tau)-\bar{g}]}{\left\{\sum_{t}\left[f(t)-\bar{f}_{\tau}\right]^{2} \sum_{t}[g(t-\tau)-\bar{g}]^{2}\right\}^{\frac{1}{2}}},
$$

where $f$ is the image, $\bar{g}$ is the template mean, $\bar{f}_{\tau}$ is the mean of $f(\tau)$ in the region under the template, and

$$
\bar{f}_{\tau}=\frac{1}{N} \sum_{t=\tau}^{\tau+N-1} f(t)
$$


The denominator represents the variance of the zero-mean image, $f(t)-\bar{f}_{\tau}$, and the shifted zero-mean template $g(t-\tau)-\bar{g}$. Dividing by these values makes $\gamma$ independent of the amplitudes of the template and image (Briechle and Hanebeck, 2001). The normalization makes the correlation coefficient more robust than using other methods for determining similarity (Briechle and Hanebeck, 2001).

Although $\gamma$ a reasonable choice for comparing two signals, it is computationally expensive. Recall that the length of $f$ and $g$ are $M$ and $N$ respectively. For each time $\tau \in\{0,1, \ldots M-N\}$, the mean image under the template region $\bar{f}_{\tau}$, and the energy of the zero mean image $e_{f}(\tau)=\sum_{t=\tau}^{\tau+N-1}\left[f(t)-\bar{f}_{\tau}\right]^{2}$ must be calculated resulting in $N(M-N+1)$ additions and $N(M-N+1)$ multiplications (Briechle and Hanebeck, 2001, Lewis, 1995). Instead of making these computations directly, we can make modifications to the method in order to reduce computing power. Consider the numerator of $\gamma$ :

$$
\gamma^{n u m}(\tau)=\sum_{t}\left[f(t)-\bar{f}_{\tau}\right][g(t-\tau)-\bar{g}]
$$

Let $f^{\prime}(t)=f(t)-\bar{f}_{\tau}$ and $g^{\prime}(t)=g(t)-\bar{g}$, we have

$$
\gamma^{n u m}(\tau)=\sum_{t} f^{\prime}(t) g^{\prime}(t-\tau)
$$

This is the definition of convolution of the image $f$ and the reversed template $g^{\prime}(-t)$ (Lewis, 1995). As such,

$$
\Longrightarrow \gamma^{\text {num }}(\tau)=\mathcal{F}^{-1}\left[\mathcal{F}\left(f^{\prime}\right) \mathcal{F}^{*}\left(g^{\prime}\right)\right],
$$

where $\mathcal{F}$ is the Fourier transformation and $\mathcal{F}^{*}$ is the complex conjugate transformation.

Extending the calculation of the numerator of $\gamma$ into the frequency domain by use of the Fast Fourier Transform (FFT) reduces the number of calculations to $12 M \log _{2} M$ multiplications and $18 M \log _{2} M$ additions/subtractions (Briechle and Hanebeck, 2001, Lewis, 1995). With $M>>$ the complexity of the direct $\gamma$ calculation is $N \cdot M$ multiplications and additions. The FFT method becomes most efficient when $\mathrm{N}$ approaches the size of $\mathrm{M}$ and when $\mathrm{N}$ and $\mathrm{M}$ are both large (Lewis, 1995). 


\subsection{Preliminary processing}

\subsubsection{Picking phase arrivals and isolating template events}

We start with a set of known template events and continuous data (see Section 3.1, Figure 3.1). In order to apply the matched-filter method, we need to extract the template waveforms from each station in the network at which the event is well recorded. We use phase arrival times (picked manually) in order to determine how much of the waveform to extract, in order to define our template window. We extract a 70 second long event waveform from every station and component within our network (Table 3.1). The long event waveform starts 10 seconds before and extends 60 seconds beyond the event origin time (Figure. 3.3).

Next, we bandpass filter the waveforms between 2 and $12 \mathrm{~Hz}$ and determine Pand S-phase arrivals at each station for each template event. The lower limit of the bandpass filter was chosen to eliminate noise below $2 \mathrm{~Hz}$; however, Du et al. (2004) notes that the method of cross-correlation presented here should not be sensitive to the filtering bounds. Each phase is manually picked to yield the most accurate phase arrival. P-phase arrivals were picked on the vertical component and an S-phase arrivals on the clearer horizontal component. Although this does not take shear wave splitting into account, because of noise levels it was more accurate to pick on a single component. A quality marker is also assigned along with the time for each pick. A quality marker denotes how confident we are in the pick (integer 0 through 4 , with 0 being the most confident). We also designate whether the phase arrival is impulsive or emergent and whether the P-phase arrival has upward or downward first motion. Although not every station gave a clear recording of a particular event, we only proceed with template generation using events that are well-recorded on at least five stations (i.e. with a pick grade $\leq 3$ ).

The next step is to select the window size used for the template waveforms. Using too short a window results in an over-abundance of false detections, whereas using too long of a window of the template waveform will result in missed detections (Baisch et al., 2008, Lees, 1998). We use a window that includes both P-and Sphase arrivals to focus on finding families of detections that have similar waveforms (Du et al., 2004, Lees, 1998). The matched-filter methodology we are using requires that a constant window be chosen for each station across the network (Brown et al., 2008, Chamberlain et al., 2014, Shelly et al., 2007). We elect to use a minimal 

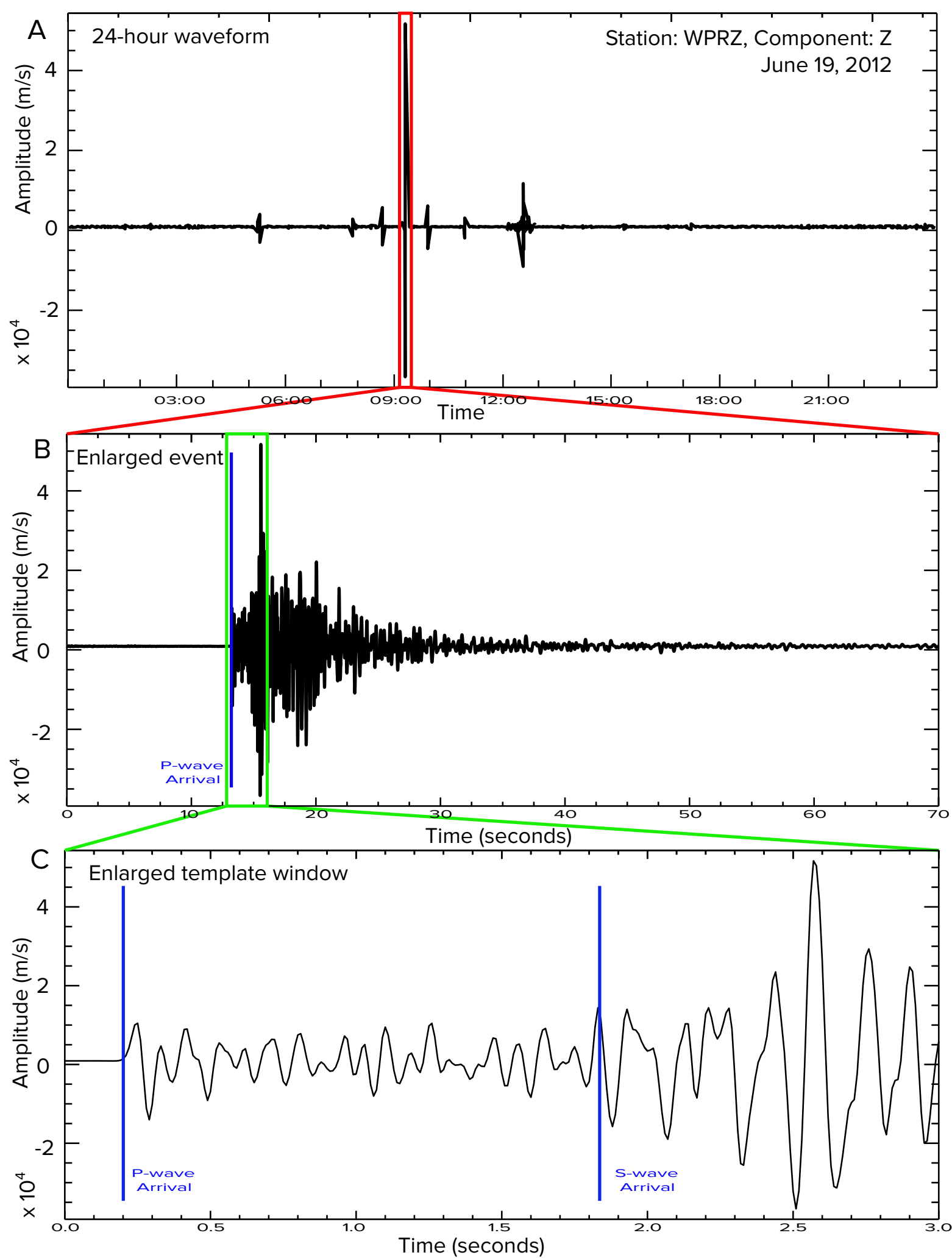

FiguRE 3.3: Diagram illustrating how a window is selected for a template at a particular station and channel (WPRZ, Vertical). For the event, a P-phase arrival is chosen, then a 3 -second window is cut out around the arrival starting 0.2 seconds before the P-phase arrival and extending 2.8 seconds afterwards. The S-phase arrival is also displayed, although not picked on this component. 
window that includes both P- and S-phase arrivals. In order for the window to contain these for all events at all stations, we select a 3 second window that starts 0.2 seconds before the P-phase arrival. We can use such a short window because of the small station-event distances, the maximum of which is approximately $5 \mathrm{~km}$, corresponding to and S-P interval of approximately 2.8 seconds. Figure 3.3 shows the template window selection for the vertical component of a template event.

\subsection{Matched-filter methodology}

Based on the understanding of the theory underpinning cross-correlation of a single time series template with a single time series image at a particular time (Section 3.2 ), we now outline how we apply it to two months of seismic waveform data and for all stations that recorded a template event. As noted above, the matchedfilter procedure we use was originally developed by Shelly et al. (2007) and further developed by Chamberlain et al. (2014). The following is a detailed description of the matched-filter process, illustrated in Figure 3.4. For convenience, we define the term 'station-component' to mean, 'each component at each station.' For example, instead of 'for every component at every station...', we state, 'for every station-component...'.

1. Template Creation: The waveform for each station-component has been cut to a 3 second window to include both P- and S-phase arrivals. Each station does not have the same P-phase arrival, thus each station may have a different window start time (Figure 3.5). It is important to take this delay into account during cross correlation so that we detect events with similar differential travel times. Delay times are calculated for each station. For station $i$, denoted $\operatorname{sta}_{i}$, its associated delay time, delay ${ }_{i}$, is equal to the difference in window start time between $\mathrm{sta}_{i}$ and the station with the earliest arrival. In other words, let a template event be well recorded on $n$ stations and

$$
\begin{aligned}
S & =\left\{t_{i} \mid t_{i}=\text { window start time for station } i, i=1,2, \ldots, n\right\} \\
\text { Let } t_{\text {min }} & =\min (S) \\
\Longrightarrow t_{\text {delay }} & =t_{i}-t_{\text {min }}
\end{aligned}
$$




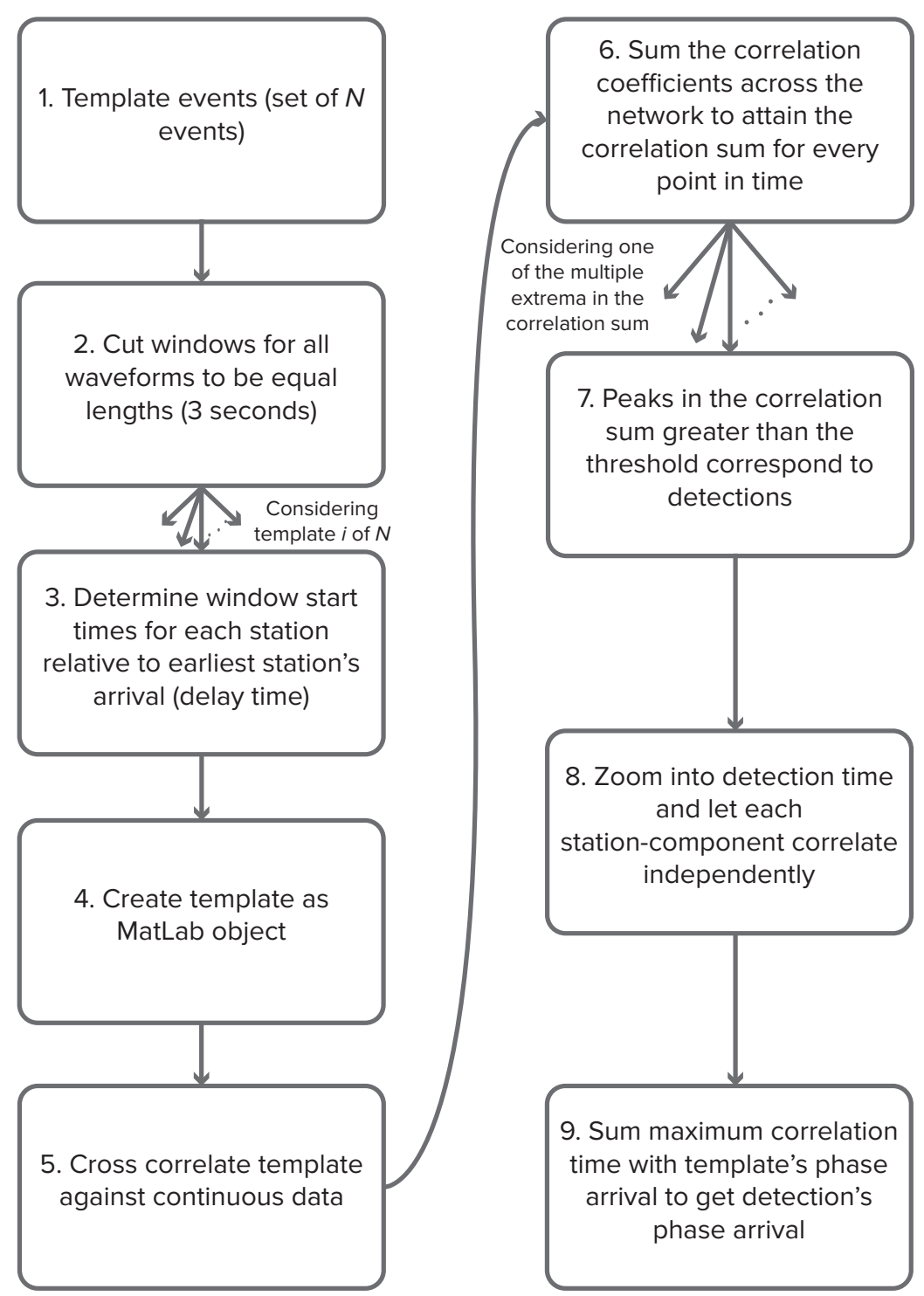

FIgURE 3.4: Flow diagram detailing the steps to perform the matched-filter method

We store the delay times, the filter range over which we bandpass (2-12 $\mathrm{Hz}$ ), the sample rate, and the waveform data for each station-component for use during the cross-correlation stage. The waveform data are stored in an $m \times n$ matrix, where $m$ is the number of stations multiplied by the number of components per station, and $n$ is the number of samples in the waveform for each component. In our case, we are using a 3 second window down-sampled to $100 \mathrm{~Hz}$ when necessary. If the earthquake is well recorded on all 11 available stations, the matrix representing the waveform data will have dimensions $33 \times 300$. 


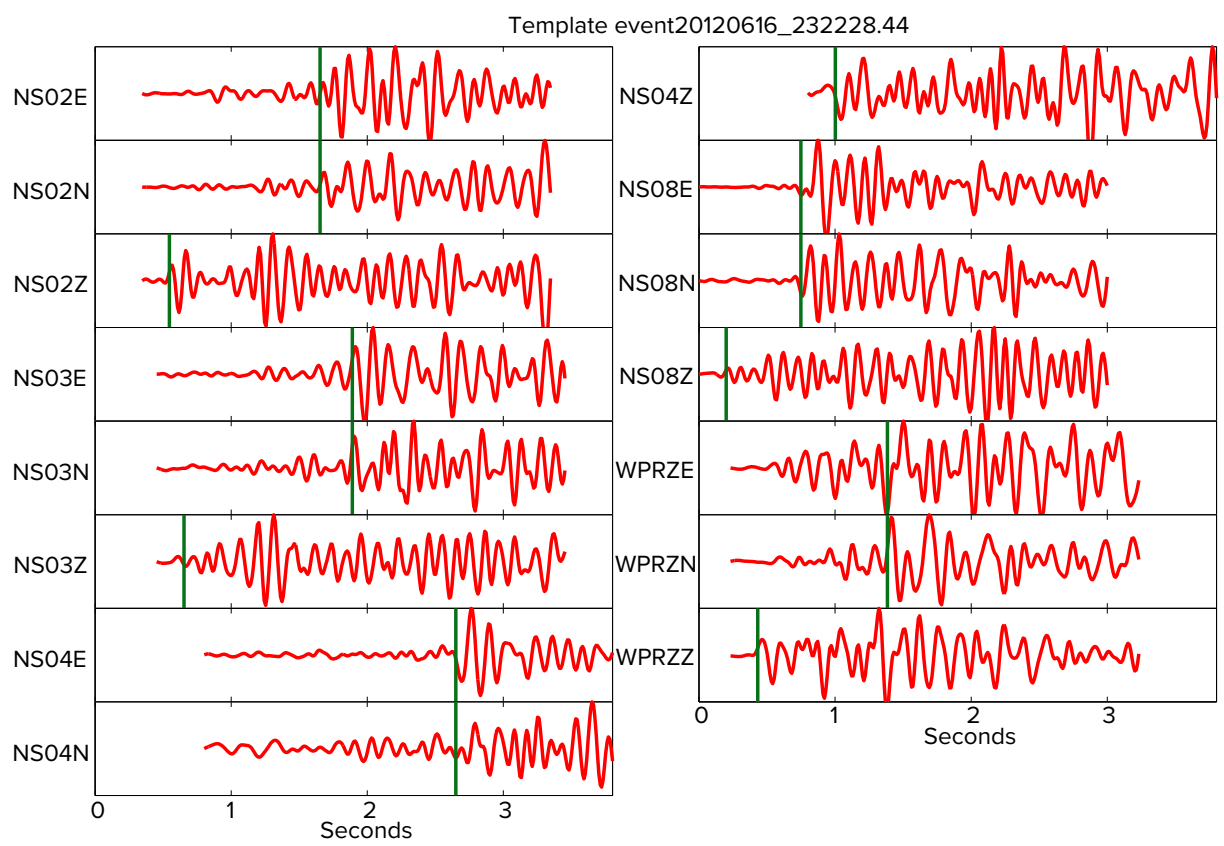

FiguRE 3.5: Example of a template waveform (event20120616_232228.44) on all its well recorded stations. Templates are named by their origin time and date (YYYYMMDD_hhmmss.ss). The pertinent phase arrival (P-phase for vertical components and S-phase for horizontal components) is marked in green. In this example, station NS08 has a delay time of zero, as it is the nearest station to the event in this case.

2. Cross-correlation against continuous data: We determine if the template signal lives within the continuous data at any point in time (besides when the template itself occurred). We adapt the normalized crosscorrelation calculation (from Section 3.2) to be performed on each stationcomponent. For each station-component, we read in the continuous waveform data for each day at that component and cross-correlate it with the respective component in the template matrix. We correlate the template with the continuous waveform data at every possible 3 second window, thus calculating the correlation coefficient at each point in time. So each correlation moves one sample further in time. The number of cross-correlation coefficients (ccc) calculated is equal to the number of samples in a day (number of seconds in a day $\times$ sample rate, or 86,400 seconds $\times 100 \frac{\text { samples }}{\text { second }}=$ 8, 640, 000 samples).

We now have a vector of correlation coefficients for each station-component. To determine whether or not we have a detection at any point in time, we consider the network correlation coefficient, or correlation sum (Brown et al., 
2008, Chamberlain et al., 2014, Shelly et al., 2007):

$$
\operatorname{cccSum}=\sum_{i=0}^{L} \operatorname{ccc}_{i}
$$

where $\mathrm{ccc}_{i}$ is the correlation coefficient for station-component $i$ of the $L$ station-components that recorded the template event.

3. Determining a daily threshold value: Before we can say whether or not we have any times in the network correlation sum that correspond to detections, we need to compute a threshold for cccSum, above which a network correlation sum value corresponds to a detection. The threshold we use is based on the median absolute deviation (MAD) of the cccSum across a day (Brown et al., 2008, Chamberlain et al., 2014, Shelly et al., 2007). The median absolute deviation (MAD) is a statistic more robust to outliers than the standard deviation, i.e. the MAD is influenced less by outliers (Brown et al., 2008, Chamberlain et al., 2014, Shelly et al., 2007). MAD is a measure of the distribution of a set of real numbers around its median (Brown et al., 2008, Chamberlain et al., 2014, Shelly et al., 2007). Specifically:

$$
\begin{aligned}
\text { Let } n_{s t a} & =\text { number of station-components, } \\
x & =\left\{x_{j}: x_{j} \in[-s, s], j=1,2, \ldots, 8,640,000\right\} \\
\text { and } \bar{x} & =\operatorname{median}(x) \\
\Longrightarrow \operatorname{MAD}(x) & =\operatorname{median}\left(\left\{y_{j}: y_{j}=\left|x_{j}-\bar{x}\right| \forall x_{j} \in x\right\}\right)
\end{aligned}
$$

We define our match-filter threshold $T$ as

$$
T=R \times \operatorname{MAD}(\operatorname{cccSum})
$$

where $R \in \mathbb{Q}$ is determined empirically (See Section 3.5).

4. Identifying detection: Given a cccSum threshold of $T$, we define a detection as the set of upper and lower bounds to the set $(-T, T)$ that are contained in cccSum.

$$
\begin{aligned}
& \text { Let } D=\text { set of detections } \\
& \Longrightarrow D=\left\{x_{i}: x_{i} \in \operatorname{cccSum} \cap\left|x_{i}\right| \geq|T|\right\}
\end{aligned}
$$




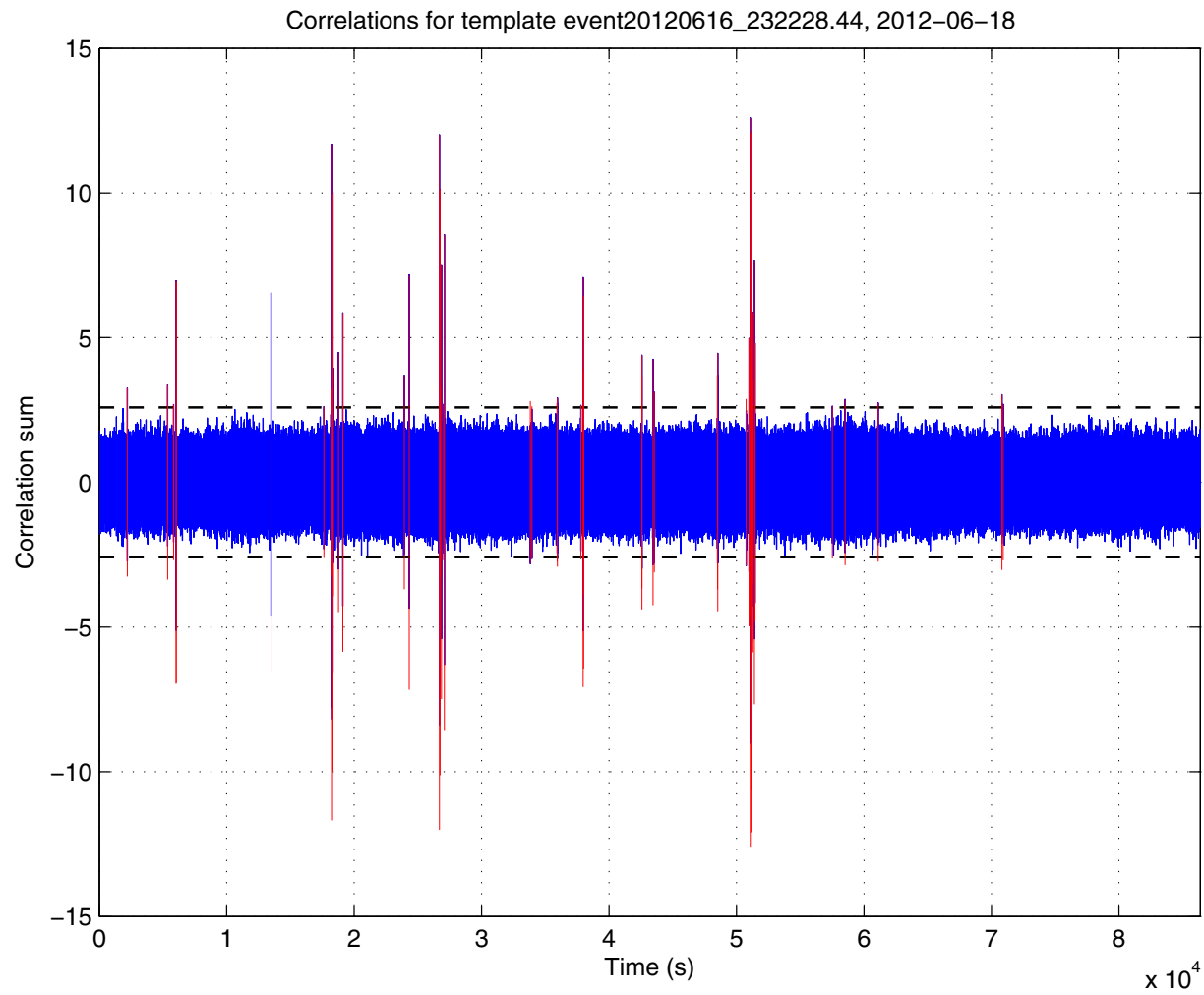

Figure 3.6: Correlation sum time series (blue) during 18 June 2012 for a template occurring on 19 June 2012. Detections are based on a matched-filter threshold of $7.6 \times \mathrm{MAD}$. Red highlights the detection times, where the crosscorrelation sum exceeds the matched-filter threshold.

In other words, detections are the extrema of the correlation coefficient sum that exceed the threshold. The detection time is a point in time when there is an extrema in the correlation sum (Figure 3.6). This does not necessarily correspond to the time when each station-component best correlates to the continuous data, due to slight differences in locations and travel times of the template and the detection. This means that the detection time approximately equals the time at which the station-component with $t_{\text {delay }}=0$ best correlates with the continuous data. The other station-components are best

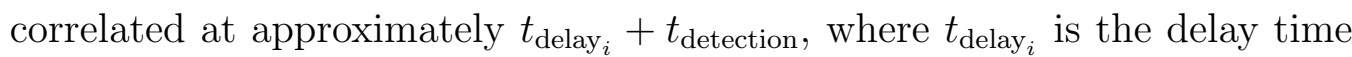
for station-component $i$.

One constraint we put on detections for each template is that they must be at least 3 seconds apart. That is, if we have a peak in the correlation sum above the threshold, then that template cannot detect another event for 3 seconds afterwards (Chamberlain et al., 2014, Shelly et al., 2007). We impose this constraint so that we do not achieve false detections from later 
parts of the coda of a detection. Three seconds is chosen as it is the length of the template.

5. Calculating detection phase arrival times: In order to calculate the differences in travel times between the different station-components, we perform a second, smaller scale cross-correlation at each detection time for each day. For a particular detection time $t_{\text {detection }_{0}}$, we allow each station-component to correlate independently within an interval centered around $t_{\text {detection }_{0}}+t_{\text {delay }_{i}}$. Each template event is $3 \mathrm{~s}$ long, so we choose a $4.5 \mathrm{~s}$ window of continuous data over which to correlate the template (Figure 3.7) (Chamberlain et al., 2014). We chose this length to minimize the amount of time the window is allowed to search over, therefore avoiding false phase picks, yet still allowing the station-component waveform sufficient independence (Chamberlain et al., 2014). For each station-component we identify the maximum correlation coefficient over this window and the time at which it occurs.

Detections typically have a lower signal to noise ratio (SNR) than the template. Consequently, stations that record the template well, may not record low SNR detections well (i.e. the noise is too strong for the earthquake signal to be well recorded, possibly due to low magnitude $M_{L}$ ). In order to deal with this problem, we only accept maximum correlation times from the secondary correlation that correspond to correlation coefficients exceeding 0.45 , based on testing through this work and on the work by DeShon et al.. For example, in Figure 3.7, the P-phase arrival recorded by stationcomponent NS04Z would be discarded since the waveform is not well correlated enough for it to be trusted. Only accepting picks from the highly correlated station-components guarantees that the detection is well recorded on all station-components we use to generate phase arrival times.

The maximum correlation time for station-component $i, t_{\text {maxccc }_{i}}$, corresponds to the start of the template window. In order to get the relative phase arrival for that particular station-component:

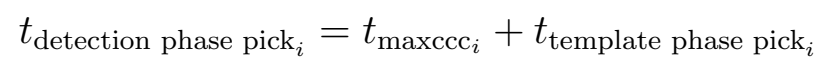

An S-file is used to store pick times for a detection and can easily be read and processed by seismic analysis program Seisan (Havskov and Ottemoller, 1999). 
NS02E 0.83

NS02N 0.87

NS02Z 0.87

NSO3E 0.88

NS03N 0.85

NS03Z 0.60

NS04E 0.60

NS04N 0.64

NS04Z 0.43

NS08E 0.91

NS08N 0.91

NS08Z 0.93

WPRZE 0.91

WPRZN 0.89

WPRZZ 0.95

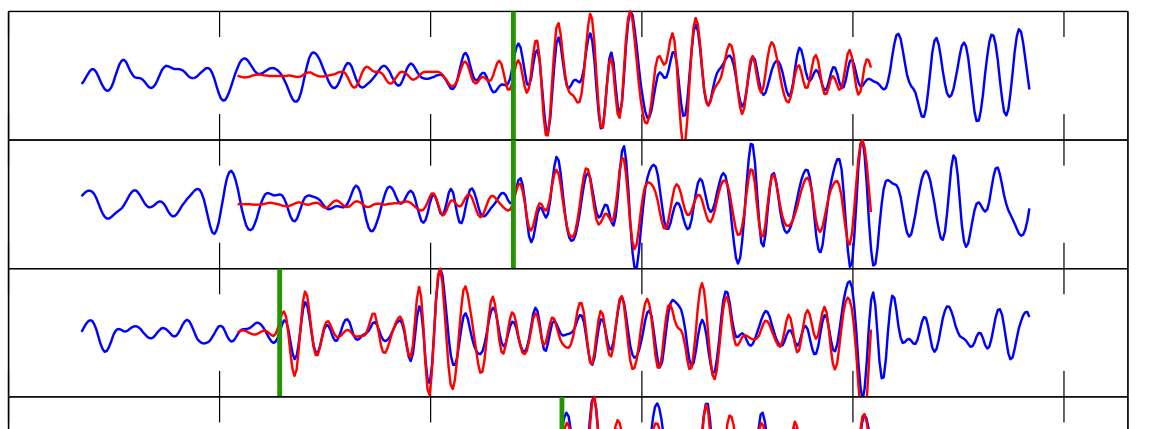

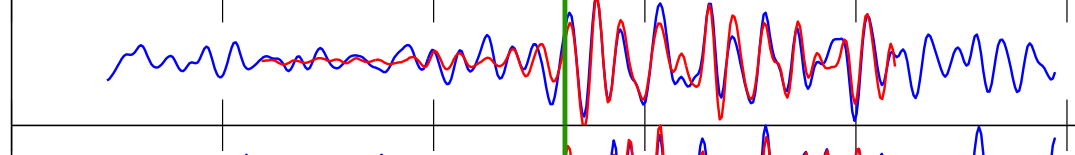

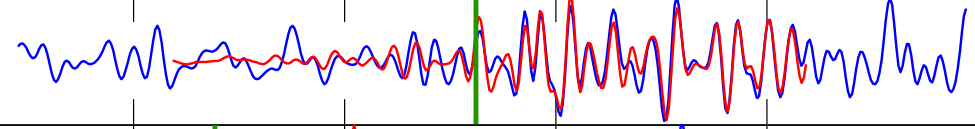

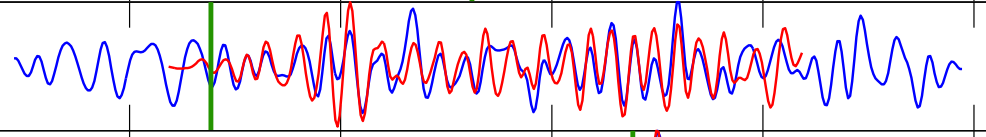

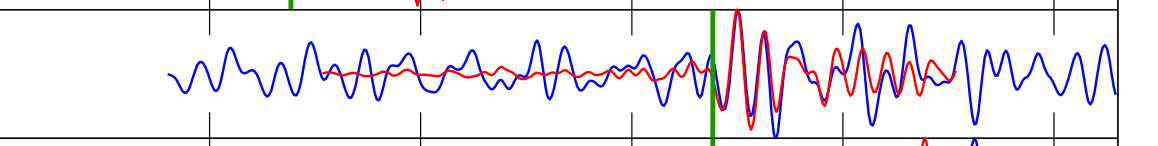

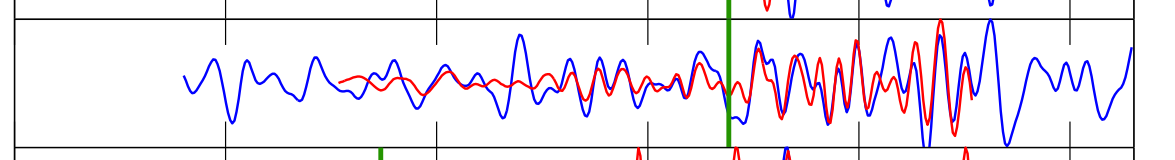

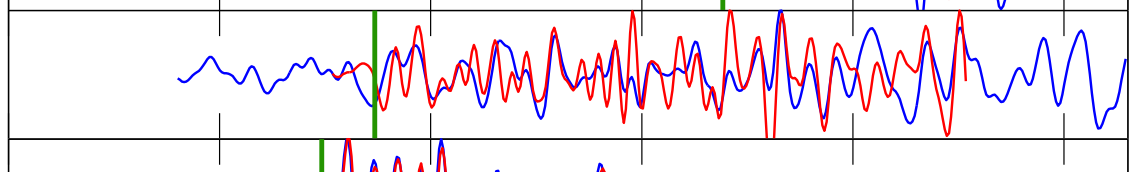

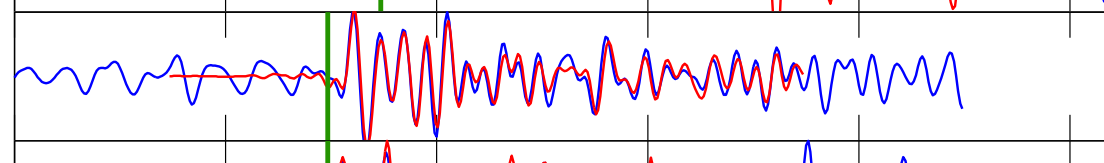

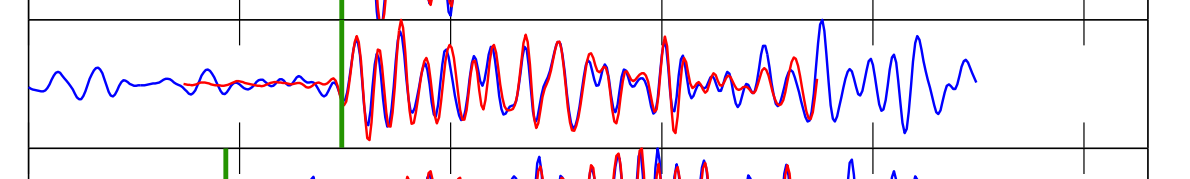

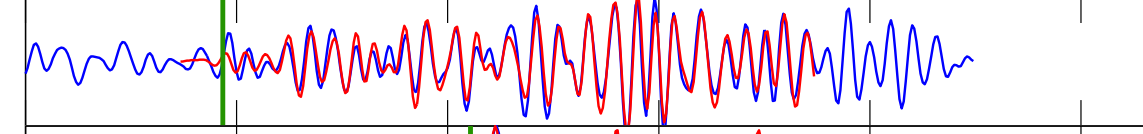

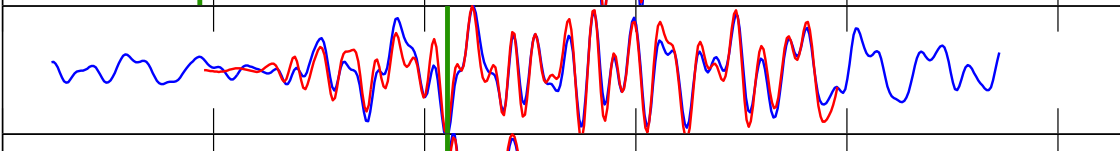

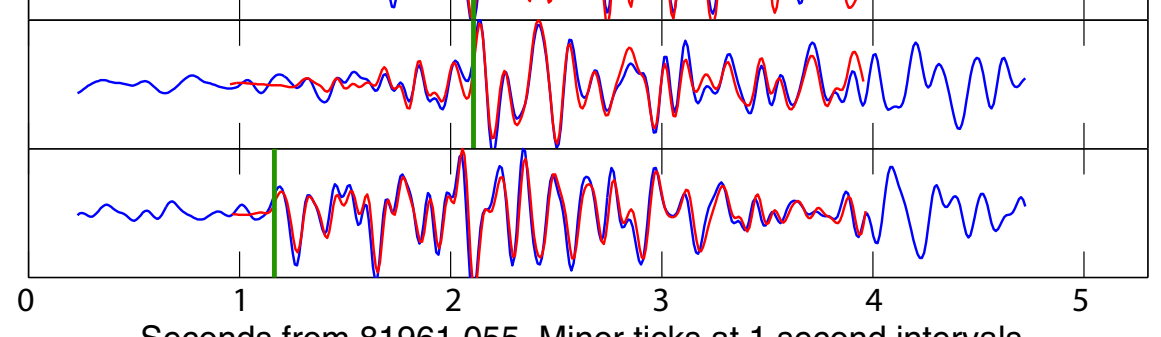

Seconds from 81961.055. Minor ticks at 1 second intervals.

FiguRE 3.7: Example of a detection from template event20120616_232228.44, 81962 seconds from the start of the day on 2012-06-15. The pertinent phase arrival (P-phase for vertical components and S-phase for horizontal components) is marked in green. The template waveform (red) is overlain on the continuous data (blue) at the point of maximum correlation for each station-component. Waveforms for station NS04 is the latest phase arrival and worst correlated. Phase picks were not used for correlation coefficients less than 0.45. Correlation coefficients are shown on the left next to the respective station names. 


\subsection{Threshold testing}

Recall from Section 3.4, step 3 that the matched-filter threshold is based on the median absolute deviation of the correlation sum throughout the day, or $R \times$ MAD (Brown et al., 2008, Chamberlain et al., 2014, Shelly et al., 2007). As explained above, we define the MAD for set $X=\left\{X_{i}\right\}$ to be MAD $=\operatorname{median}_{i}\left(\left|X_{i}-\operatorname{median}(X)\right|\right)$ (Shelly et al., 2007). For each day that matched-filtering is performed, we presume the threshold may vary due to daily variation of correlation sum MAD, but the $R$ value remains constant. The $R$ value is a function of the seismic noise levels of our study area and the templates that we are using. We determine the $R$ value through a synthetic testing process (Chamberlain et al., 2014, Rawlinson, 2011, Shelly et al., 2007). The synthetic test involves seeding varying sizes of template waveforms into phase-randomized noise to create a new day-long waveform. Then, using the same matched-filter routine described in Section 3.4, we search through this constructed waveform at increasing $R$ values and record when we get successful detections of seeds and when we get false detections (Chamberlain et al., 2014, Rawlinson, 2011, Shelly et al., 2007). We require an $R$ value that results in a threshold for which we have $95 \%$ confidence that a detection is true.

To begin the synthetic test, we start with a template and continuous waveform data for the day the template occurred. We use the day of the template because we know we can achieve a detection for this template at the noise amplitude on this day (Figure 3.8) (Chamberlain et al., 2014, Shelly et al., 2007). For each stationcomponent, we randomize the phase of the continuous waveform in order to prevent getting a true detection from an actual event that lives within the waveform for the given day (Chamberlain et al., 2014, Rawlinson, 2011, Shelly et al., 2007). We perform the phase randomization in the frequency domain by considering the imaginary component of the fast Fourier transform of the continuous waveform, randomizing the phase component, then performing the inverse Fourier transform. In other words, for continuous waveform $f$,

$$
\begin{aligned}
F & =\mathcal{F}(f) \\
r & =|F| \text { is the magnitude of the spectrum-amplitude component of } f \\
\phi & =\arctan \frac{\operatorname{Im}(F)}{\operatorname{Re}(F)} \text { is the phase component of } f
\end{aligned}
$$



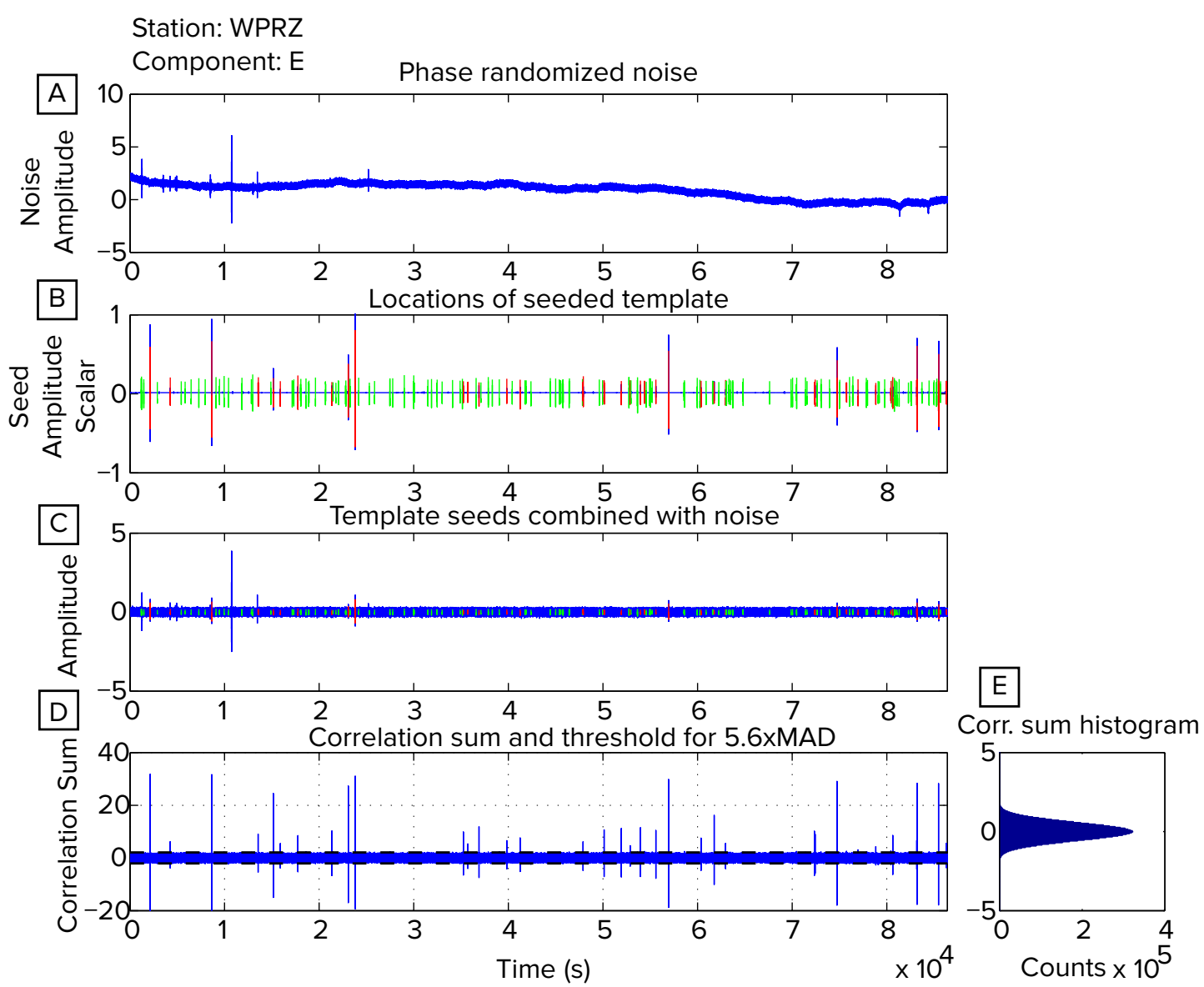

FIGURE 3.8: An example of the synthetic testing for the matched-filter threshold multiplier for template on 10/6/2012 showing (A) the phase randomized noise $f_{\text {rand }},(\mathrm{B})$ the location and scaled amplitudes of the seeds $\left(g^{\prime}(t-\tau)\right),(\mathrm{C})$ the convolution of seeds and the noise $\left(g^{\prime}\left(t-\tau_{i}\right)+f_{\text {rand }}\right),(\mathrm{D})$ the correlation sum with threshold of 5.6× MAD in dashed line, and (E) a histogram of correlation sum values according to their frequency. In (B) and (C) the red highlighted sections of the time series correspond to successful detections, where as green highlights correspond to false detections.

Let there be $N$ distinct phases. We randomize the phase vector, denoted $\phi_{\text {rand }}$ and combine it with the magnitude $r$.

$$
\begin{gathered}
\phi_{\text {rand }}=\left\{\phi_{\text {rand }, j}: \phi_{\text {rand }, j} \text { is a random scalar between } 0 \text { and } 1, j=1,2, \ldots, N\right\} \\
\phi_{\text {rand }, j}=\frac{\phi_{\text {rand }, j}-\bar{\phi}}{\phi_{\max }-\bar{\phi}} \times \phi_{\max } \\
\phi_{\text {rand }, \frac{N}{2}+1}=\phi_{\frac{N}{2}+1}
\end{gathered}
$$

This sets the nyquist frequency of the randomized phase to that of the original.

$$
\begin{aligned}
& F_{\text {rand }}=r \times e^{i \phi_{\text {rand }}} \\
& f_{\text {rand }}=\mathcal{F}^{-1}\left(F_{\text {rand }}\right)
\end{aligned}
$$


Next we create a set of scalar multiples of the template that will be seeded at random times throughout the randomized noise $f_{\text {rand }}$. We use 130 seeds, 10 of whose amplitudes are scaled by a value between 1 and 0.1, 30 of whose amplitudes are scaled by a value between 0.1 and 0.01 , and 90 of whose amplitudes are scaled by a value between 0.01 and 0.001 . We multiply the template's amplitude by a scalar for each seed and then place the seed at a random time inside the day long noise (Figure 3.8). The following describes the placing of a seed of template $g$.

For the $i^{\text {th }}$ seed event, let $\lambda_{i} \in(0,1]$ be a random scalar and $\tau_{i} \in[0,86400]$ be a random time, in seconds, from the beginning of the day. Let $g^{\prime}(t)=\lambda_{i} \cdot g(t)$. Then, consider the Dirac delta function $\delta\left(t-\tau_{i}\right)$ at time $\tau_{i}$. We convolve the scaled template and the Dirac delta:

$$
\begin{aligned}
g^{\prime}(t) * \delta\left(t-\tau_{i}\right) & =\int_{-\infty}^{\infty} g^{\prime}(T) \delta\left(t-\tau_{i}-T\right) \mathrm{d} T \\
& =\int_{-\infty}^{\infty} g^{\prime}(T) \delta\left(T-\left(t-\tau_{i}\right)\right) \mathrm{d} T \\
& =g^{\prime}\left(t-\tau_{i}\right)
\end{aligned}
$$

Finally, we sum the convolution with the phase randomized noise $g^{\prime}\left(t-\tau_{i}\right)+f_{\text {rand }}$ to get the seeded, scaled template within the noise.

For each randomization and seeding, we run though an array of threshold multiplier values $R$ ranging from 5.0 to 10.0, in increments of 0.1 . For the given threshold multiplier value $R$ we have a set of detections comprising a subset of successful detections of seeds and a subset of false detections. To determine which detections are successful, we compare the set of positions in time of the detections to the positions in time of the seeds. A detection whose position matches a position of a seed is termed a successful seed detection. The set of detections whose position in time does not match any of the seeds is the set of false detections. To prevent bias from a particular randomization or seed location, we perform this analysis 5 times for each of the 110 templates, then average the results for each threshold multiplier $R$.

We choose a particular threshold multiplier for the matched-filter threshold based on a $95 \%$ confidence that a detection is true (Figure 3.9). We require that at the chosen threshold multiplier, all templates satisfy the $95 \%$ confidence requirement. To achieve this, we settle on a multiplier $R=7.6$. 


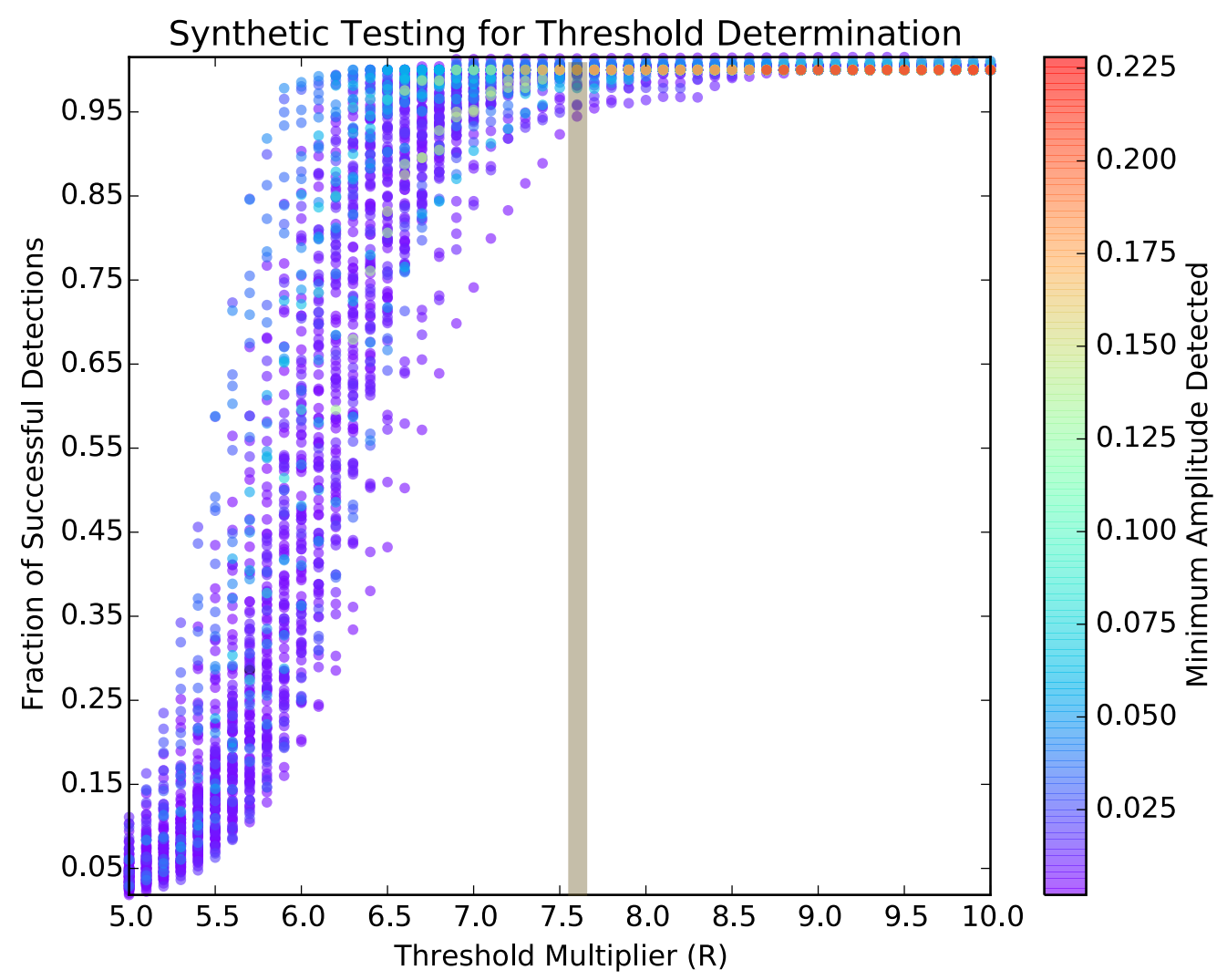

FiguRE 3.9: Results from synthetic testing for determination of matched-filter threshold scalar $R$. The scatter plot shows how the percentage of successful detections varies with increasing $R$ value. Each point represents the percentage of successful detections for one of the 110 templates at the corresponding $R$ value. The color of the point corresponds to the minimum amplitude scalar $\lambda_{\min }$ detected by that particular template at that corresponding threshold. The scale for $\lambda_{\min }$ is to the right of the graph. In order for all templates to achieve $95 \%$ successful detections, an $R \geq 7.6$ is required. The value of $R=7.6$ is highlighted in grey for reference. This corresponds to a minimum detected amplitude of at most 0.18 for all templates.

Besides the percentage of false detections, we can also calculate the detected minimum amplitude scalar for each trial of the synthetic test, i.e. $\lambda_{\min }=\operatorname{minimum}\left(\lambda_{i}\right) \forall i$. We seed such a large number of small scaled events (90 events scaled by 0.01 and 0.001) so that there are seeds with signal-to-noise values low enough that they are not detected. By seeding many small events of decreasing value, we are confident that the calculated minimum amplitude scalar is not inflated by the lack of a 'small enough' seed. 


\subsection{Multiplet construction}

It is also useful to characterize the similarity between the original template events. This is key to determining how many unique events we are actually using as templates. Our basis of constructing families, or multiplets of template events, involves taking two template events and cross-correlating all common stations against each other. Each component is cross-correlated individually. One of the wave forms from a given station-component is padded with zeros at the beginning and end to make sure that we can get the maximum correlation, and to avoid propagating errors made during the phase picking procedure. This allows one template to act as the matched filter template, and the other zero-padded template as the image.

For every possible pair of template events, we calculate the normalized correlation coefficient for common stations. The normalized cross-correlation coefficient is the network correlation sum between two template events, but normalized by the number of shared station-components. Each normalized cross-correlation coefficient is stored in an square matrix of size $110 \times 110$, where 110 is the number of template events. Each row and column corresponds to a particular template; the $i$ th row and $i$ th column representing the $i$ th template. The correlation matrix is symmetric and each diagonal entry equals 1, as this represents correlating templates with themselves (Figure 3.10).

After correlating all templates against each other, we select the templates that will be part of the first multiplet. This follows an iterative process that begins with calculating the mean correlation coefficient for each template, mcc $_{i}$. The template that has the largest mcc $_{i}$ is designated master template for the multiplet being constructed. We then look at all templates that correlate with the master template beyond a certain threshold, equal to $1.1 \times$ mcc. These are the templates that will join the master template in the current multiplet. With construction of multiplet 1 complete, we repeat this with the set of remaining events. Again, we correlate the templates against each other, then calculate the mean correlation coefficient for each template, and set the template with the maximum mean correlation coefficient as the master template for the second multiplet. We select templates which correlate better than $1.1 \times$ mcc with the second master template. Multiplet 2 comprises these templates. We continue this iterative process until we only have a set of templates that do not correlate with any other template above 


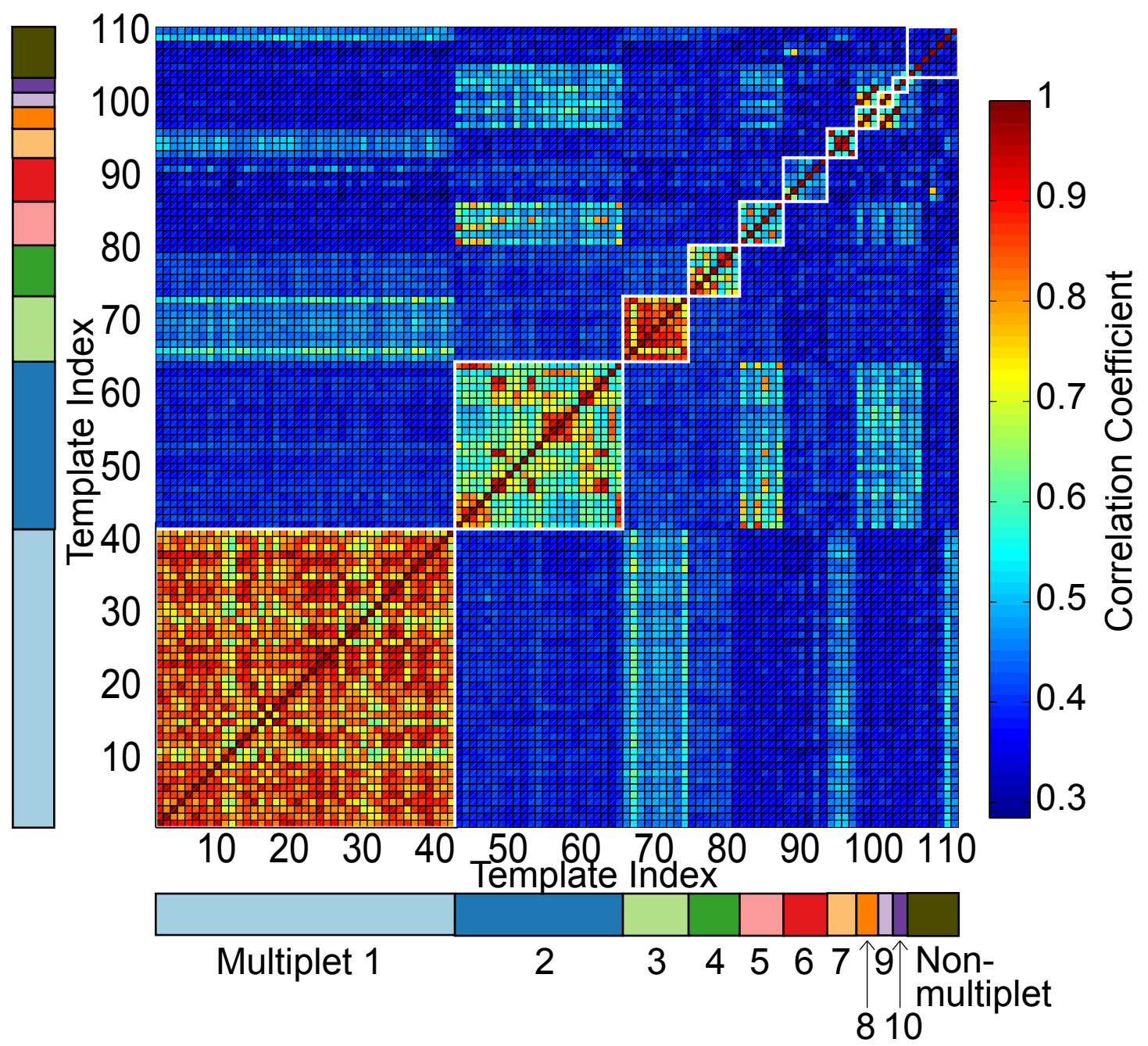

Figure 3.10: Correlation matrix of showing the 110 templates correlated against each template. Each row and respective column represents a single template, i.e. the matrix is symmetrical. For templates $i$ and $j$, the normalized network correlation coefficient is located in row $i$, column $j$ - or vice versa. Warmer colors represent higher correlation, with the red diagonal being autocorrelation with a coefficient of 1 . The white outline groups each of the multiplets to illustrate how the correlation coefficients within a multiplet compare to those excluded from it. 
$1.1 \times \operatorname{mcc}_{i}$. The threshold of $1.1 \times$ mcc is based on testing through this research. Three multipliers $(1.0 \times, 1.1 \times$, and $1.5 \times$ mcc) were tested. When qualitatively comparing the results of the three different mulitpliers, $1.1 \times$ mcc grouped the templates in the most natural way.

\subsection{Calculating local magnitude using singular value decomposition}

We calculate magnitudes for a subset of the matched-filter detections. Magnitudes for highly similar waveforms are well suited to be calculated using a method based on singular value decomposition (SVD). This method, developed by Rubinstein and Ellsworth (2010), exploits the entire waveform, as opposed to other methods which determine magnitude based on single parameters such as duration or amplitude. The SVD calculation is implemented as part of EQcorrscan, a python package developed by Chamberlain and Badger (2015) to perform matched-filtering and other related utilities. This method works regardless of frequency, so we continue to bandpass filter detection waveforms between 2-12 Hz. We ensure that waveforms from individual station-components have cross-correlation coefficients of at least 0.85 over a 3.5 second waveform in order for them to be considered in the magnitude calculation (Rubinstein and Ellsworth, 2010).

The SVD method is a way to describe a matrix $A$ by factoring it into three other matrices $U, V$, and $\Sigma$. $U$ and $V$ are sets of basis vectors for the amplitude space and the waveform space, respectively. $A$ is expressed as:

$$
A=U \Sigma V^{\prime}
$$

where $V^{\prime}$ is the transpose of $V$. $V$ is a matrix of waveforms that form a basis for the space of detection waveforms. In other words, any detection waveform in matrix $A$ can be represented as a linear combination of waveforms from $V$. When the waveform entries of $A$ are highly similar, as is the case for our data set, then most of the data comprising $A$ can be described as a scalar multiple of the first basis vector in $V$, denoted $\bar{V}_{1}$. When similarity is extremely high between events, then basis vectors besides the first will simply describe the noise of each seismogram. The relative importance of each basis vector, $\bar{V}_{i}$, is described by 
its corresponding singular value $\Sigma_{i}$. Large singular values correspond to more influential basis vectors. The relative amplitudes of each basis vector are stored in $U$. We can assume that the first basis vector describes the relative amplitude (and seismic moment) of the seismograms of $A$ due to the highly similar nature of $A$ 's waveforms (Rubinstein and Ellsworth, 2010). We repeat the decomposition of $A$ for each station-component available.

The relative amplitude for a single event may differ across the set of available components. We combine all relative moments by assuming that the weighted sum of the moments of two events at the same station is zero (Rubinstein and Ellsworth, 2010). For example, for moments $m_{1}$ and $m_{2}$ for events 1 and 2, respectively, let the relative amplitudes be 1.37 and 1.0. We can sum their relative amplitudes to make $1.37 m_{1}-1.0 m_{2}=0$, or:

$$
(1.37-1) \times\left(\begin{array}{l}
m_{1} \\
m_{2}
\end{array}\right)=d,
$$

where $d=[0]$. Extrapolating this example to all considered events, we arrive at the system equations:

$$
K B=D,
$$

where $K$ is a matrix detailing the relative amplitudes, $B$ is an unknown vector of relative moments, and $D$ is the zero vector (Rubinstein and Ellsworth, 2010). We then solve for $B$ using a least squares solution, giving us the local magnitudes of the most highly similar matched-filter detections.

\subsubsection{Gutenberg-richter distribution}

According to Gutenberg and Richter (1944), a catalog of seismicity generally follows the Gutenberg-Richter relation: $\log N=a-b M$, where $N$ is the number of events of magnitude $M$ and $a, b \in \mathbb{R}$. Figure 3.11 illustrates this relationship and depicts the best fit line for the data, with equation: $\log N=a-b M$. The b-value describes the ratio of large-magnitude to small-magnitude events within a catalogue (Woessner and Wiemer, 2005). A b-value of 1 corresponds to the expected magnitude distribution of seismicity in a tectonic setting (Gutenberg and Richter, 1944). 


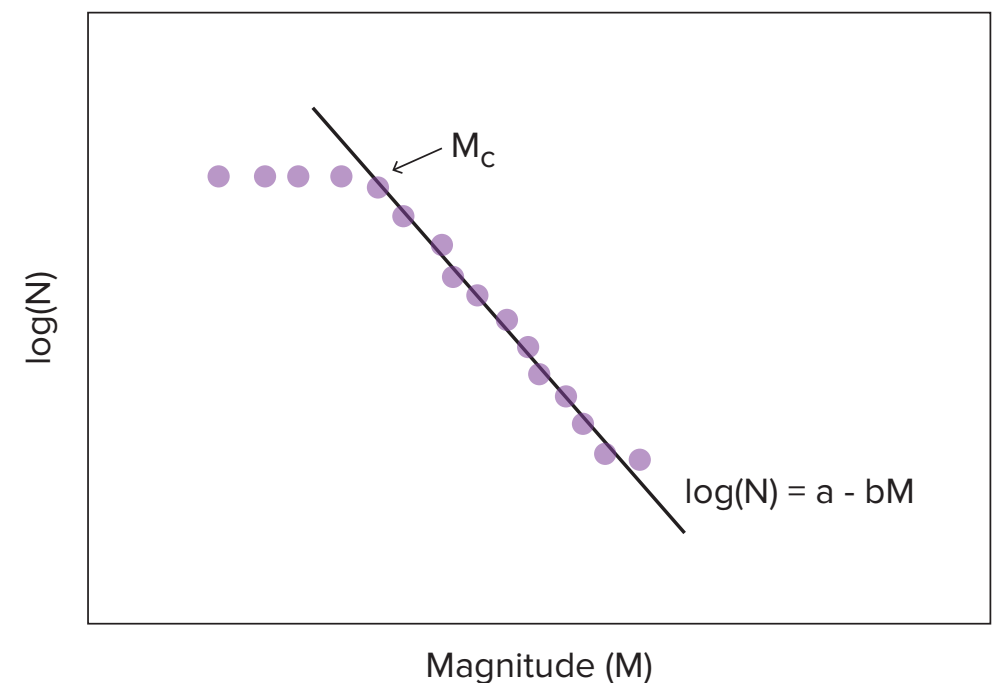

FiguRE 3.11: Example of the Gutenberg-Richter relation for a catalog of earthquakes. The points represent the frequency of a given magnitude event. The line follows the equation $\log N=a-b M$ for magnitudes above $M_{c}$

At small magnitudes the the log-linear relationship between magnitude and frequency with constant b-value no longer applies (Woessner and Wiemer, 2005). This is ascribed to catalogue incompleteness. In other words, not enough lowmagnitude events are detected (due to low SNR) in order to have a complete representation of the catalogue. A catalogue is considered complete for magnitudes for which the $\log N=a-b M$ relationship holds (Woessner and Wiemer, 2005); the minimum magnitude for which this relationship holds is the completeness magnitude, $M_{c}$. Woessner and Wiemer (2005) give several methods for calculating $M_{c}$. We use a method that determines $M_{c}$ by calculating the maximum curvature of the graph of $\log N$ against $M$ (Figure 3.11). This calculation is performed by computing the maximum value of the first derivative of the b-value. In other words, we would calculate the maximum second derivative of the frequency magnitude curve (Woessner and Wiemer, 2005)

\subsection{Double-difference relocation}

Poupinet et al. (1984) developed a method to calculate precise relative locations for spatially clustered seismic events, known as double-difference relocation. Earthquake location is classified by hypocenter ( $\mathrm{x}, \mathrm{y}, \mathrm{z}$ - usually latitude, longitude, depth) and an origin time (t). These parameters are determined by seismic wave arrival, typically from multiple stations and phases. With knowledge of seismic 
velocities at depth (a velocity model), we can estimate the travel time of a wave passing through various layers of differing velocity (Figure 3.12, Table 3.2) Sherburn and Bourguignon (2013).

Using a matched-filter method to detect new events results in clusters of events that have highly similar waveforms. This produces circumstances that are ideal for using a double-difference location method. Microearthquakes are assumed to have similar waveforms due to similar locations, source parameters, and focal mechanisms. The HypoDD program uses a double-difference method, which uses small difference in travel times between events to calculate small differences in location (Figure 3.13) (Waldhauser and Ellsworth, 2000). Figure 3.14 illustrates how double-difference relocations can add significant resolution to earthquake locations (Poupinet et al., 1984, Waldhauser and Ellsworth, 2000). The double-difference method gives relative locations of earthquakes based on the fact that the sourcereceiver ray paths of two events are very similar when their hypocenter locations are similar (Poupinet et al., 1984). This implies that any difference in travel time is caused by spatial variation at the source, and not by velocity heterogeneities along the ray paths (Waldhauser and Ellsworth, 2000).

TABLE 3.2: 1 dimensional P-wave velocity model for Ngatamariki (Zara Rawlinson, pers. com. 2015)

\begin{tabular}{cc}
\hline Top of layer $(\mathrm{km}$ above sea level) & P-wave Velocity $(\mathrm{km} / \mathrm{s})$ \\
\hline 1 & 1.5 \\
0.2 & 2 \\
0 & 2.5 \\
-0.1 & 3 \\
-0.2 & 4 \\
-0.7 & 4.5 \\
-1.1 & 4.7 \\
-1.3 & 5 \\
-2.7 & 5.3 \\
-3.7 & 5.5 \\
-5 & 6 \\
-22 & 7.4 \\
\hline
\end{tabular}

Waldhauser and Ellsworth (2000) took advantage of differential travel times from cross-correlation of waveforms and/or standard travel time differences from catalogue phase arrivals. The double-difference method works by minimizing residuals of event pairs at each recording station using a least squares (LSQR) approach 


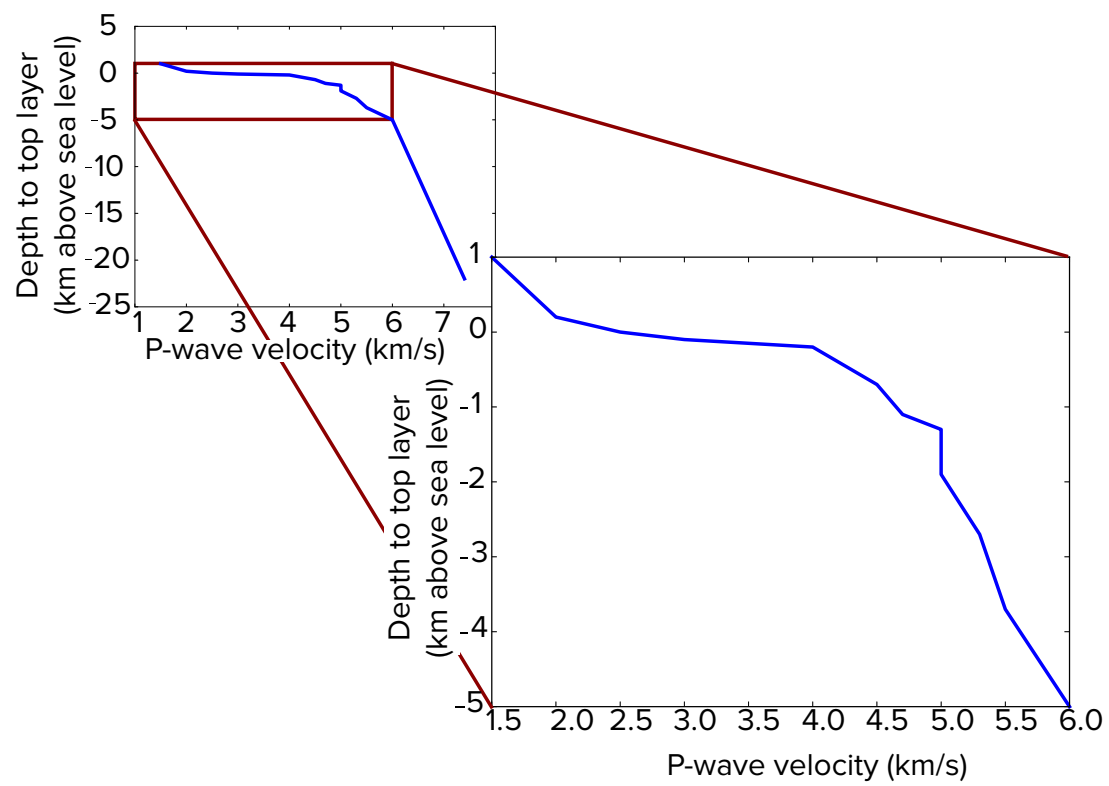

FiguRE 3.12: A 1D Ngatamariki velocity model constructed with data from a down hole check-shot survey of injection well NM9 (Figure 1.4) (Zara Rawlinson ,pers. comm., 2015) .

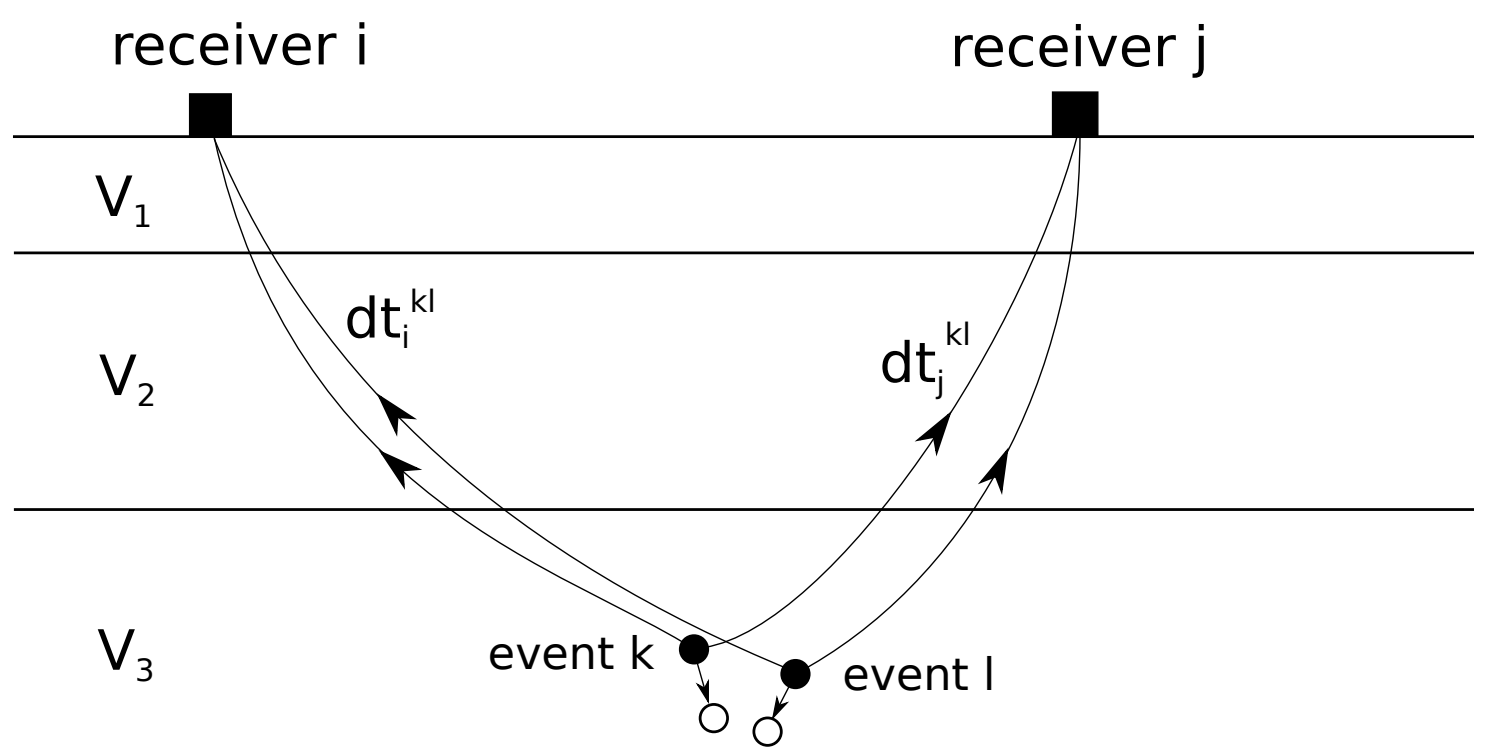

Figure 3.13: Schematic of the double-difference algorithm. The initial locations (solid circles) and relocations (open circles) are shown with the corresponding travel times differences $d t_{i}^{k l}$ (difference in travel time for events $k$ and $l$ at station $i$ ) via the ray path shown in solid line. The relocation vector is shown between the original and relocated hypocenters. 

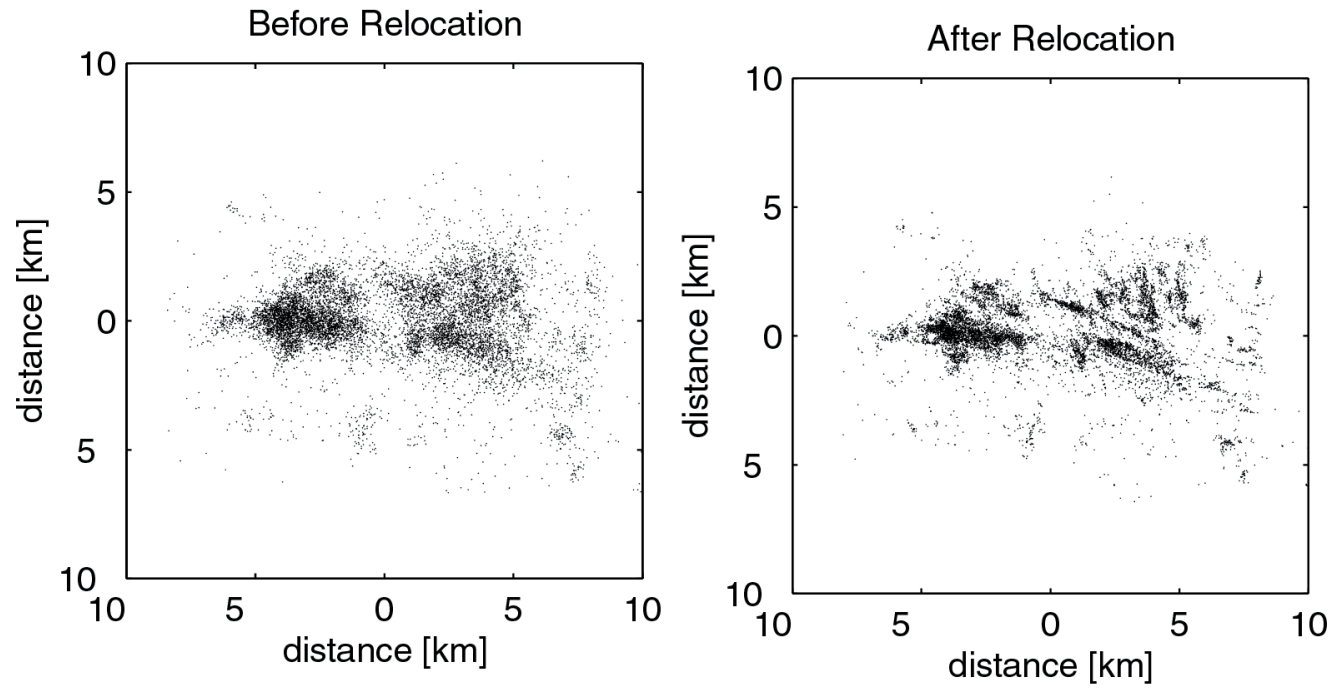

FiguRE 3.14: An example of improvement in resolution that can be achieved with HypoDD (Waldhauser and Ellsworth, 2000).

or singular value decomposition (SVD). Although the SVD method is more robust and estimates uncertainties to a higher degree, the LSQR method is more appropriate for larger data sets (> 100 events) since the LSQR approach is less computationally expensive than the SVD approach (Waldhauser and Ellsworth, 2000).

The residual, $r$, is the difference between observed $\left(t^{o b s}\right)$ and calculated $\left(t^{c a l}\right)$ travel times. For an event $k$ measured at station $i, r_{i}^{k}=\left(t^{o b s}-t^{c a l}\right)_{i}^{k}$. Residual minimization reduces to a linear differential equation (Waldhauser and Ellsworth, 2000):

$$
\frac{\partial t_{i}^{k}}{\partial \mathbf{m}} \delta \mathbf{m}^{k}=r_{i}^{k}
$$

where $\delta \mathbf{m}^{k}=\left(\delta x^{k}, \delta y^{k}, \delta z^{k}, \delta \tau^{k}\right)$ is the vector of perturbations in space $(x, y, z)$ and origin time $(\tau)$ of the event's hypocenter. The difference in residuals between a pair of events $k$ and $l$ (denoted $d r_{i}^{k l}$ ) is:

$$
d r_{i}^{k l}=\left(t_{i}^{k}-t_{i}^{l}\right)^{o b s}-\left(t_{i}^{k}-t_{i}^{l}\right)^{c a l}
$$

Taking the difference of equation (3.1) for event $k$ from the synonymous version for event $l$, we see that

$$
\frac{\partial t_{i}^{k}}{\partial \mathbf{m}} \delta \mathbf{m}^{k}-\frac{\partial t_{i}^{l}}{\partial \mathbf{m}} \delta \mathbf{m}^{l}=d r_{i}^{k l}
$$


TABLE 3.3: HypoDD parameter definitions

\begin{tabular}{cc}
\hline Parameter & Meaning \\
\hline MINWGHT & min. pick weight allowed \\
MAXDIST & max. distance in km between event pair and stations \\
MAXSEP & max. hypocentral separation in km \\
MAXNGH & max. number of neighbors per event \\
MINLNK & min. number of links required to define a neighbor \\
MINOBS & min. number of links per pair saved \\
MAXOBS & max. number of links per pair saved \\
IDAT & Type of data used. $2=$ catalogue times \\
IPHA & Phase types used. $1=$ P; $2=$ S; $3=$ P\&S \\
DIST & max. dist [km] between cluster centroid and stations \\
OBSCC, OBSCT & min. \# of obs/pair for cross-corr. or catalogue data, respectively \\
ISTART & Starting source for locations. $2=$ from network sources \\
ISOLV & Method to solve system of equations. 2= LSQR \\
WTCTP, WTCTS & Weights for catalogue P and S arrivals, respectively \\
WRCT & residual threshold weight \\
WDCT & max. distance [km] between pairs of events \\
DAMP & damping factor for speed of solving LSQR method \\
\hline
\end{tabular}

Waldhauser and Ellsworth constructed a system of these equations for all possible event pairs, resulting in:

$$
W G m=W d,
$$

where $\mathrm{G}$ is an $M \times 4 N$ matrix comprising the partial derivative information, such that $M$ is the number of observations and $N$ is the number of events. $d$ is a vector with the double-differences and $m$ is a vector of length $4 N$ comprising the changes in hypocentral parameters $\delta \mathbf{m}$. $W$ is a diagonal matrix of weights for each equation. Waldhauser and Ellsworth (2000) extended this system of equations by adding the condition that

$$
\sum_{k=1}^{N} \delta \mathbf{m}^{k}=0
$$

The user has control of the parameters used to determine precise relative locations. The key parameters control the clustering (MAXNGH, MINLINK, MINOBS, MAXSEP, OBSCC, and OBSCT), the method that the system of equations is solved (ISTART and ISOLV), the weighting parameters for P- or S-phase arrivals (WTCTP and WTCTS), residual and distance cutoff factors (WRCT and WDCT), and LSQR damping factor (DAMP) (Tables 3.3, 3.4 and 3.5). For more in-depth description of the parameters, see Waldhauser and Ellsworth (2000). 
TABle 3.4: Parameters used for ph2dt

\begin{tabular}{ccccccc}
\hline MINWGHT & MAXDIST & MAXSEP & MAXNGH & MINLNK & MINOBS & MAXOBS \\
\hline 0 & 200 & 2 & 1000 & 10 & 9 & 33 \\
\hline
\end{tabular}

TABLE 3.5: Parameters used for HypoDD earthquake relocation

\begin{tabular}{ccccccc}
\hline IDAT & IPHA & DIST & OBSCC & OBSCT & ISTART & ISOLV \\
\hline 2 & 1 & 20 & 0 & 3 & 2 & 2 \\
\hline Iterations & WTCTP & WTCTS & WRCT & WDCT & DAMP \\
\hline 5 & 1.0 & -9 & 4 & 4 & 100 \\
5 & 0.9 & 0.1 & 3 & 3 & 95 \\
5 & 0.8 & 0.2 & 3 & 3 & 90 \\
5 & 0.7 & 0.3 & 2 & 2 & 85 \\
\hline
\end{tabular}




\section{Chapter 4}

\section{Results}

\subsection{Template multiplets}

We quantify the similarity between all 110 template events by calculating normalized network cross-correlation coefficients (Section 3.5). As an example, Figure 4.1 shows cross-correlated templates 1 and 2 plotted against each other. Alternatively, Figure 4.2 shows an example of two templates that are poorly correlated across their shared stations.

Figure 3.10 shows the matrix of the normalized network correlation coefficients from all 110 templates. We divide these templates, based on their correlation coefficients, into 10 multiplets and one "group" of non-multiplet templates (those that did not match well enough to be in any group). The non-multiplet events are located throughout the study area (Figure 4.3).

At the Ngatamariki field, multiplets are spatially clustered in three areas, two of which are in proximity to injection wells (Figure 4.3). There are four multiplets (1, 3, 4 and 7 ) in the northern part of the field (Figure 4.3). Multiplet 1 (42 templates) is the closest to NM8, whereas multiplets 3 (nine templates) and 4 (seven templates) are slightly further. Multiplet 7 (four templates) sits north of multiplet 1. Although multiplet 3 and 4 are near each other, with some overlap in map view, the waveforms are distinct; normalized correlation coefficients between templates in multiplet 3 and 4 do not exceed 0.45 .

Multiplet 2 (23 templates) dominates the southern extent of the field near well NM10; however, the events are not as well correlated within the multiplet as those 
of multiplet 1 to the north. Multiplets 5 (six templates), 8 (three templates), and 10 (two templates) are also located near multiplet 2. Although we do not consider them part of the same multiplet, Figure 3.10 shows that the waveforms in multiplets 5,8 , and 10 are more similar to template waveforms in multiplet 2 than when comparing intermultiplet templates from multiplets 1, 3, 4, and 7 .

We identify two other multiplets (6 and 9) that they are located outside of Ngatamariki and within the Rotokawa field. These events are poorly recorded on most of the stations in the Ngatamariki network and are constrained primarily by Rotokawa stations. We nevertheless use these events as matched-filter templates in the analysis below.

We use all 110 template events in the matched-filter method, even though they are grouped by similar waveforms. This will result in templates within a multiplet detecting similar sets of events. We resolve this in Section 4.2. The reason we did not use the alternative, which would be to use just "unique" templates to avoid duplicating detections, is that we wanted to guarantee that detections were not missed. Additionally, it is pertinent to check for duplicate detections regardless of whether all template events were used in the match-filter method.

\subsection{Matched-filter detection}

We construct a time series of the correlation sum for each day from June through July for every template (Figure 3.6). This time series is compared to the threshold (dashed line in Figure 3.6) for a given template and day in order to find detections (Section 3.4). The peaks in the correlation sum that exceed the threshold (Figure 3.6, highlighted in red) correspond to detections in the seismic waveform (Figures 4.4 and 4.5). Examining the waveform for a particularly dense interval of detections, we observe detection qualities (i.e. cross-correlation coefficients) vary over a short time span (Figures 4.4 and 4.5 ).

The matched-filter template search yields hundreds of detections. A single template detects as many as 570 seismic event, or as few as 16 (Table A). On average, each template detects 237 events. Figure 4.4 and 4.5 illustrate how the correlation sum varies with the quality and amplitude of detections. We observe that high correlation values can result even with small amplitude detections. 
Template 1 and 2 Cross Correlation

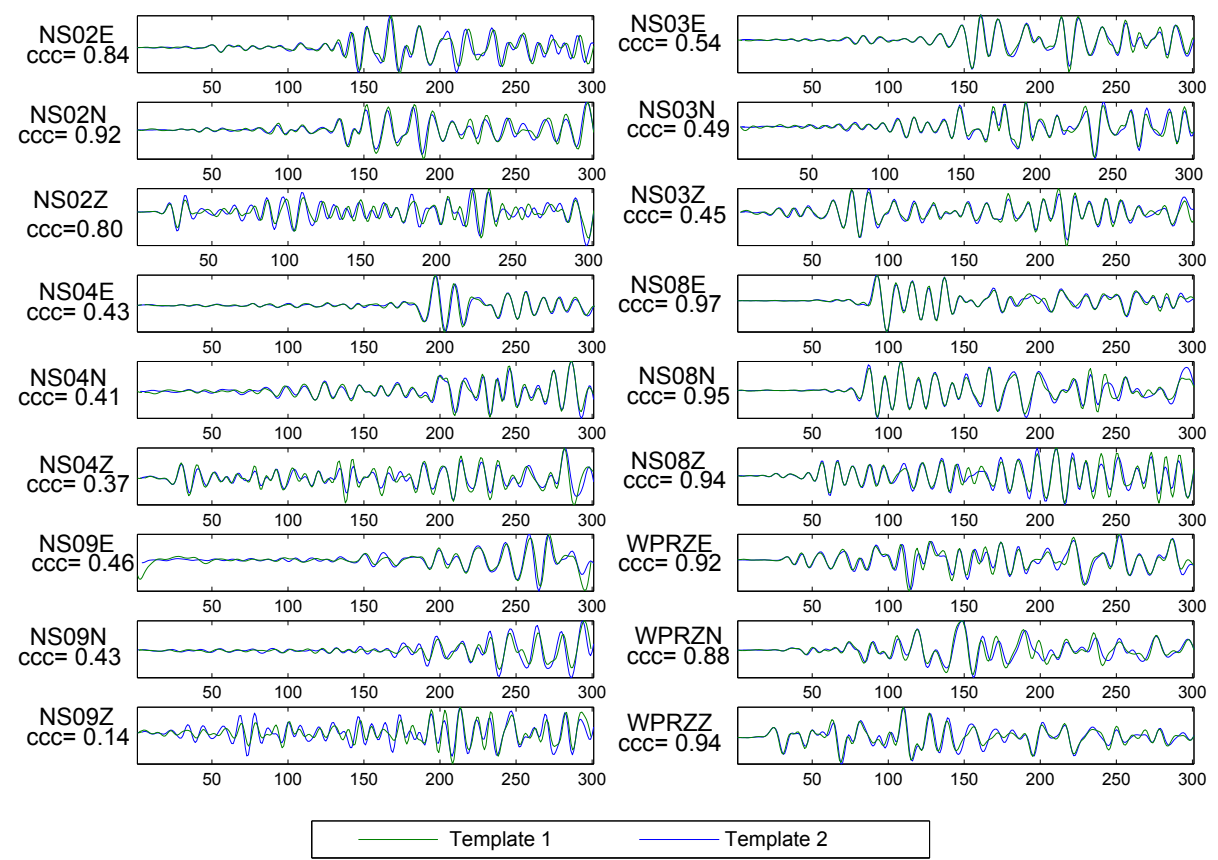

FiguRE 4.1: Example of two well correlated templates with waveforms for common stations. Correlation coefficients ( $\mathrm{ccc}$ ), station name and channel initial are displayed beside each pair of waveforms.

We also visualize the detections via a linear stack (for quality control purposes). The stacked detections are only used for a qualitative quality assurance on the matched-filter methodology and are further assessed beyond this. We linearly stack a subset of the best correlated detections in order to compare many detections from a particular template at a particular station (Figures 4.6 and 4.7 display waveforms with normalized amplitudes). Based on the ease of visual inspection, we have chosen to display $6 \%$ of the best correlated detections, which is just less than Chamberlain et al. (2014) stack of $10 \%$ used for detecting low frequency earthquakes. As expected, there is a high level of similarity between the detected waveforms at a given station-component.

One challenge with using multiple templates with similar waveforms is that multiple templates detect the same events (Figure 4.8). We see that templates from a common multiplet produce very similar detection times. Figure 4.8 illustrates that templates from the two largest multiplets ( 1 and 2$)$ detect events in clusters. This is expected given the nature of the matched-filter methodology. Although we can consider an individual template's detections, it is necessary to consider the 
Template 1 and 3 Cross Correlation

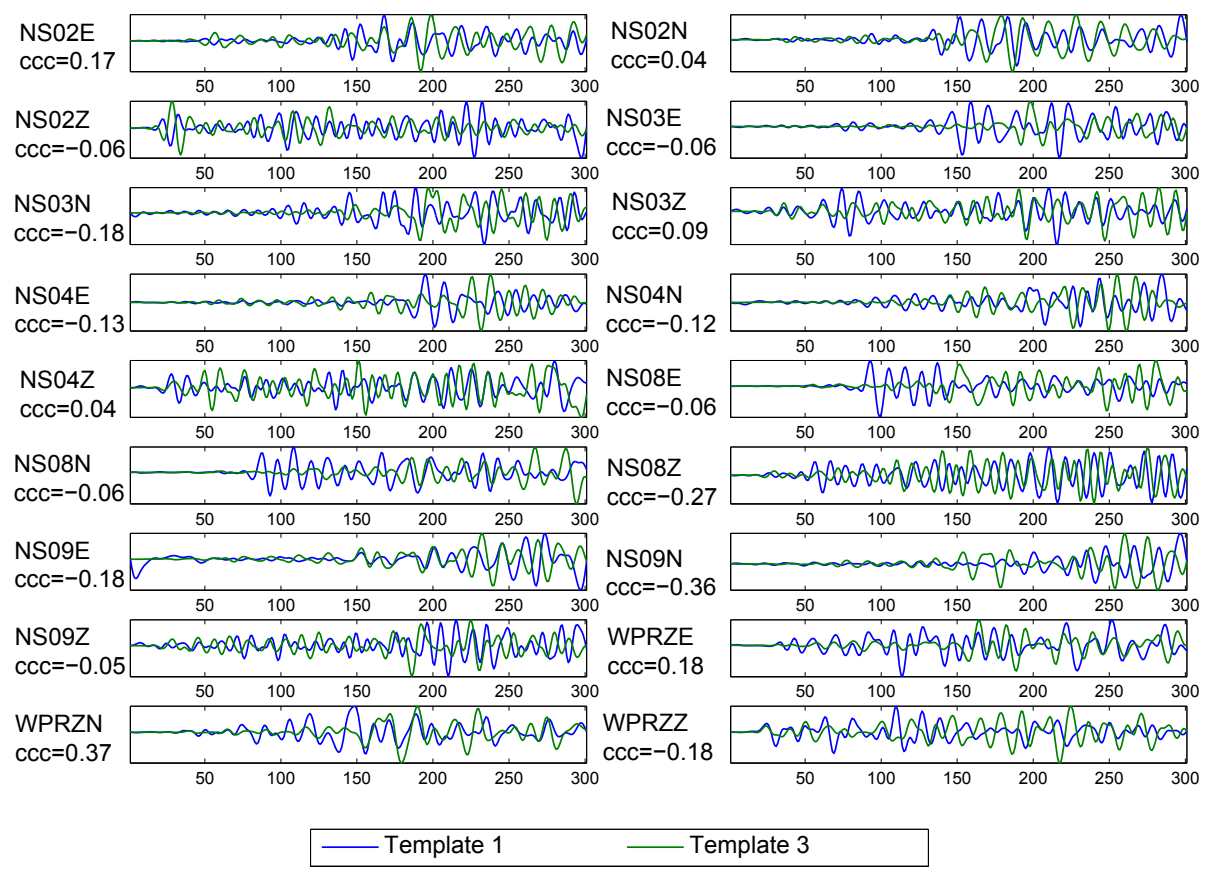

Figure 4.2: Example of two poorly correlated templates with waveforms for common stations. Correlation coefficients (ccc), station name (e.g. 'NS02') and channel (e.g. 'E') initial are displayed beside each pair of waveforms.

set of unique detections from all templates. Compiling the total overlapping set of detections into this unique set requires a method of determining whether a pair of detections are the same seismic event. We achieve this by comparing detections pairwise in the time domain. We consider two events to be the same if the are detected within 1.5 seconds of each other. For this time comparison, we use the time of maximum cross-correlation across the network. That is, let events A and $\mathrm{B}$ be detected by different templates at times $T_{A}$ and $T_{B}$, respectively,

$$
\text { Event } \mathrm{A}=\text { Event } \mathrm{B} \Longleftrightarrow\left|T_{A}-T_{B}\right| \leq 1.5 \text { seconds }
$$

For a given template, we require that detections be at least 3 seconds apart - giving confidence that our time threshold of 1.5 seconds to define unique detections is reasonable and effective. One problem with this approach however is the scenario where multiple events are truly simultaneous. The direct method of comparing all detections with a 1.5 second threshold cannot cope with the case in which we have events occurring simultaneously in different parts of the field. In this case, our method would consider these events the same. We avoid this primarily by 


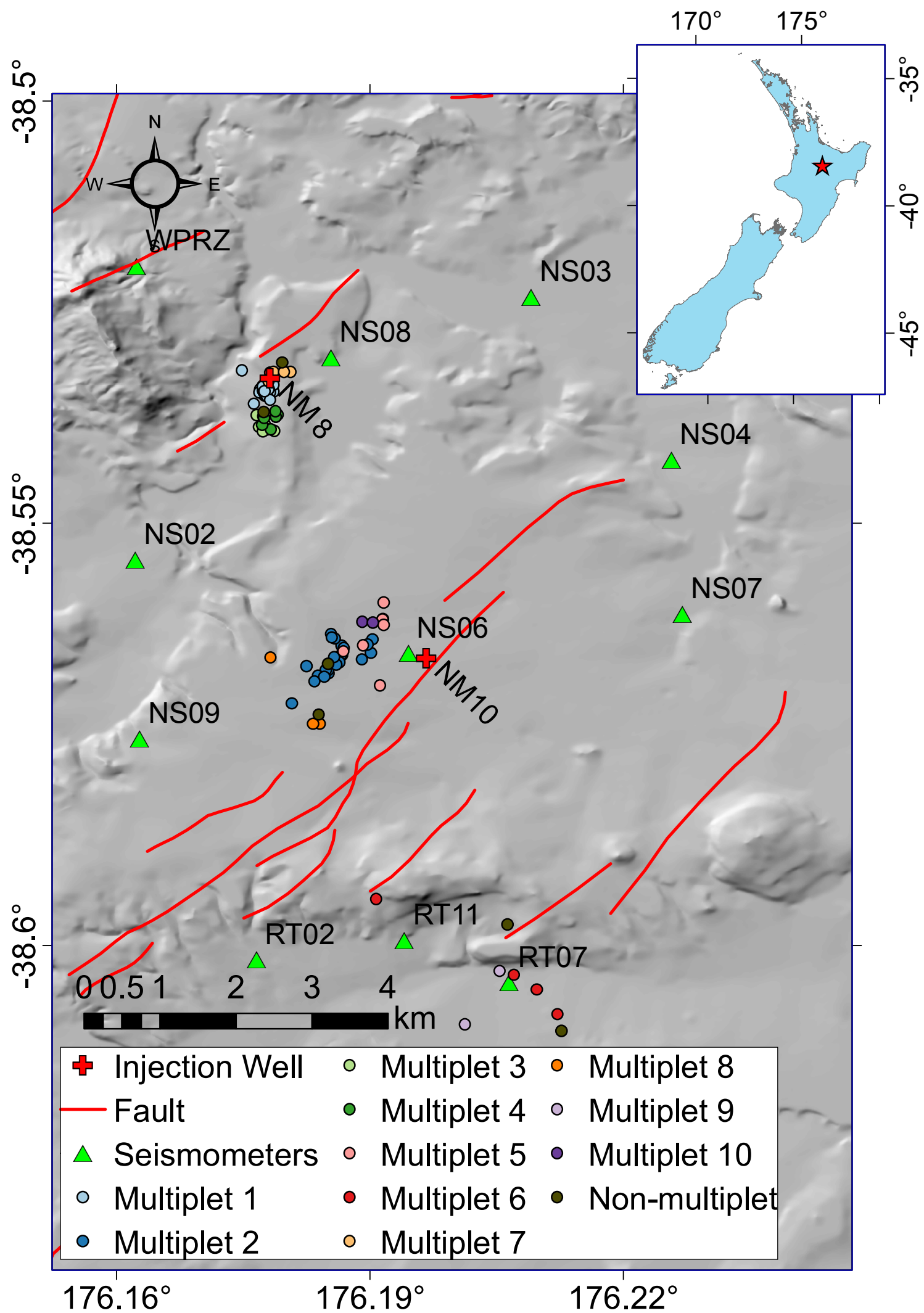

Figure 4.3: Map of the Ngatamariki geothermal field with template events clustered by multiplet. The colors of each event correspond to the multiplet it is a member of. 


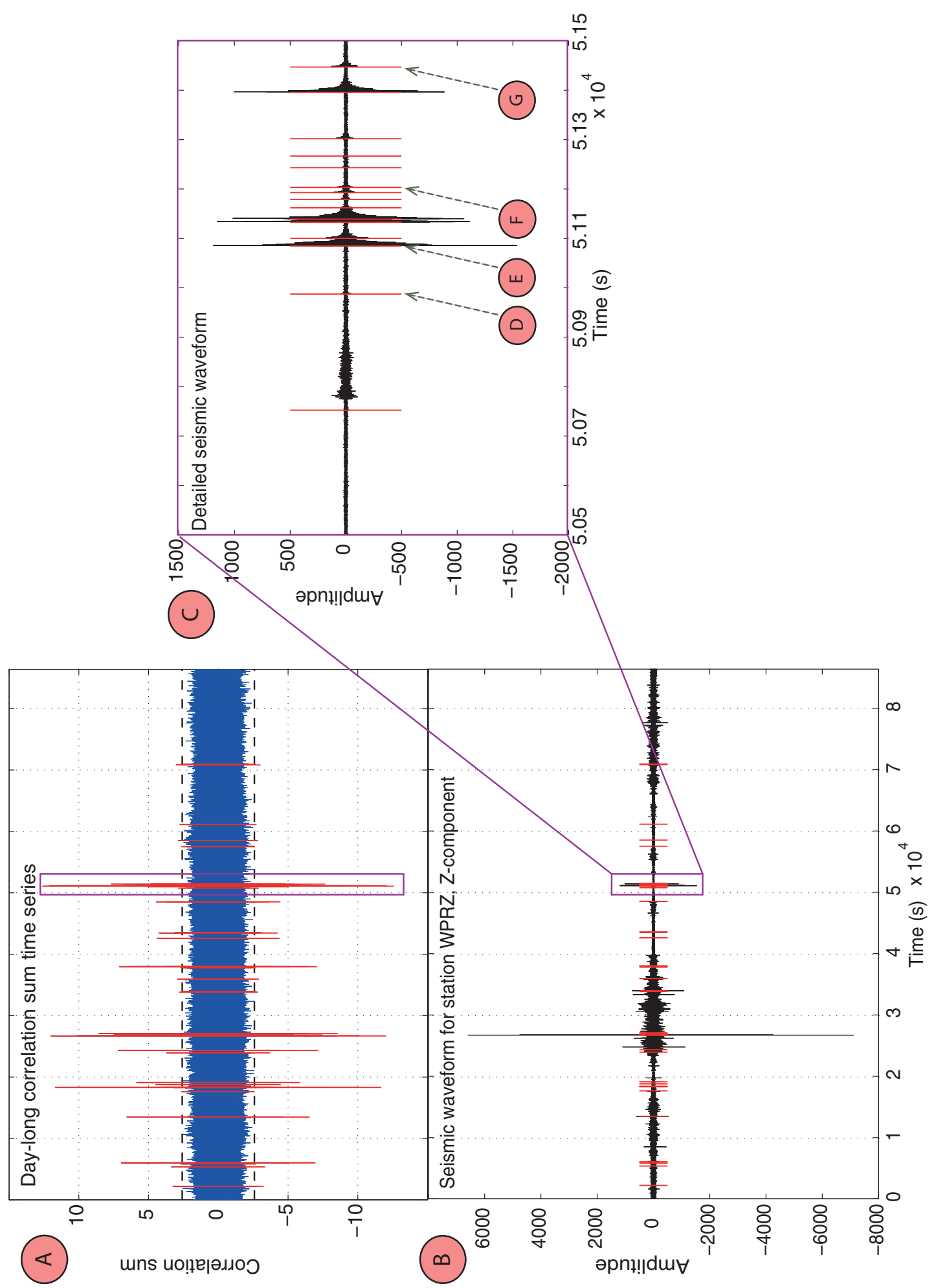

FIGURE 4.4: Enlarged area of waveform that corresponds to a time period of frequent, high correlation sum values from Figure 3.6. (A) Correlation sum time series (blue) for template occurring on 19 June 2012, with detections highlighted in red. (B) The 18 June 2012 continuous waveform from station WPRZ, component $\mathrm{Z}$ with detections highlighted in red. (C) Enlarged image of the WPRZ waveform during a time of frequent large correlation sum values. (D)-(G) See Figure 4.5 

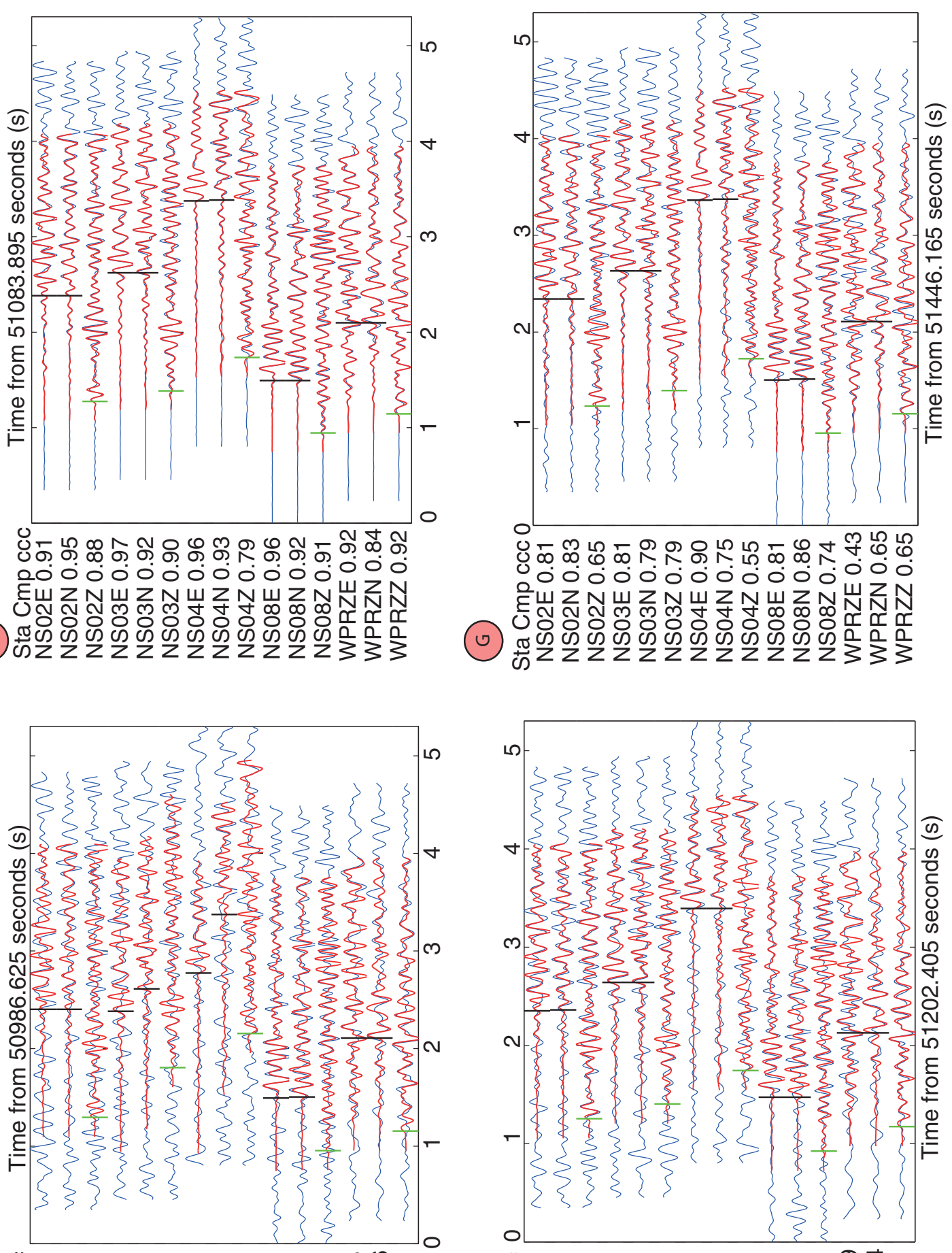

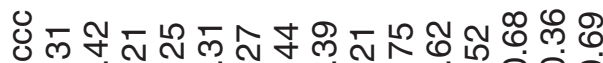
000000000000000

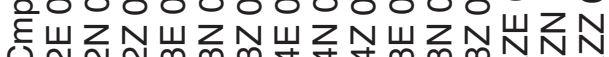

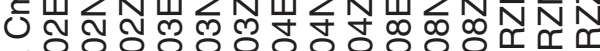
(0)

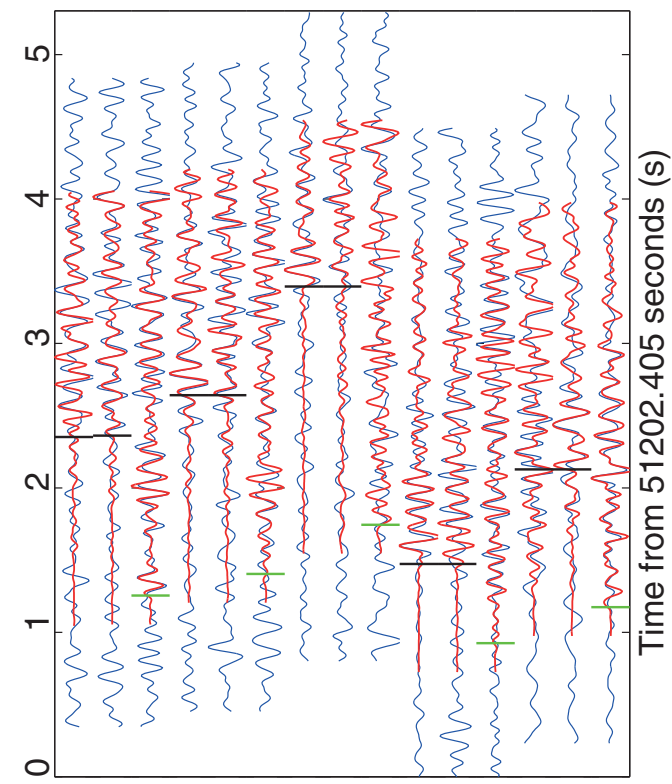

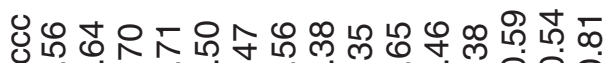
足 $0^{\circ} 0^{\circ} 0^{\circ} 0^{\circ} 0^{\circ} 0^{\circ} 0^{\circ} 0^{\circ} 0$ E

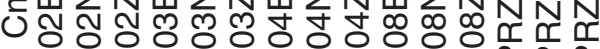

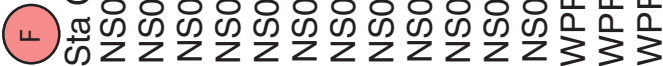

FiguRE 4.5: Detection examples from Figure 4.4. The template (red) is overlain on the continuous waveform (blue) at the time of the detection. Station (Sta), component (Cmp), and cross-correlation coefficient (ccc) are denoted to the left of each waveform. The P-phase arrivals are marked with green, while S-phase arrivals are marked in black. For $(\mathrm{A})-(\mathrm{C})$ See Figure 4.4 
Stack of event 20120616_232228.44 WPRZZ

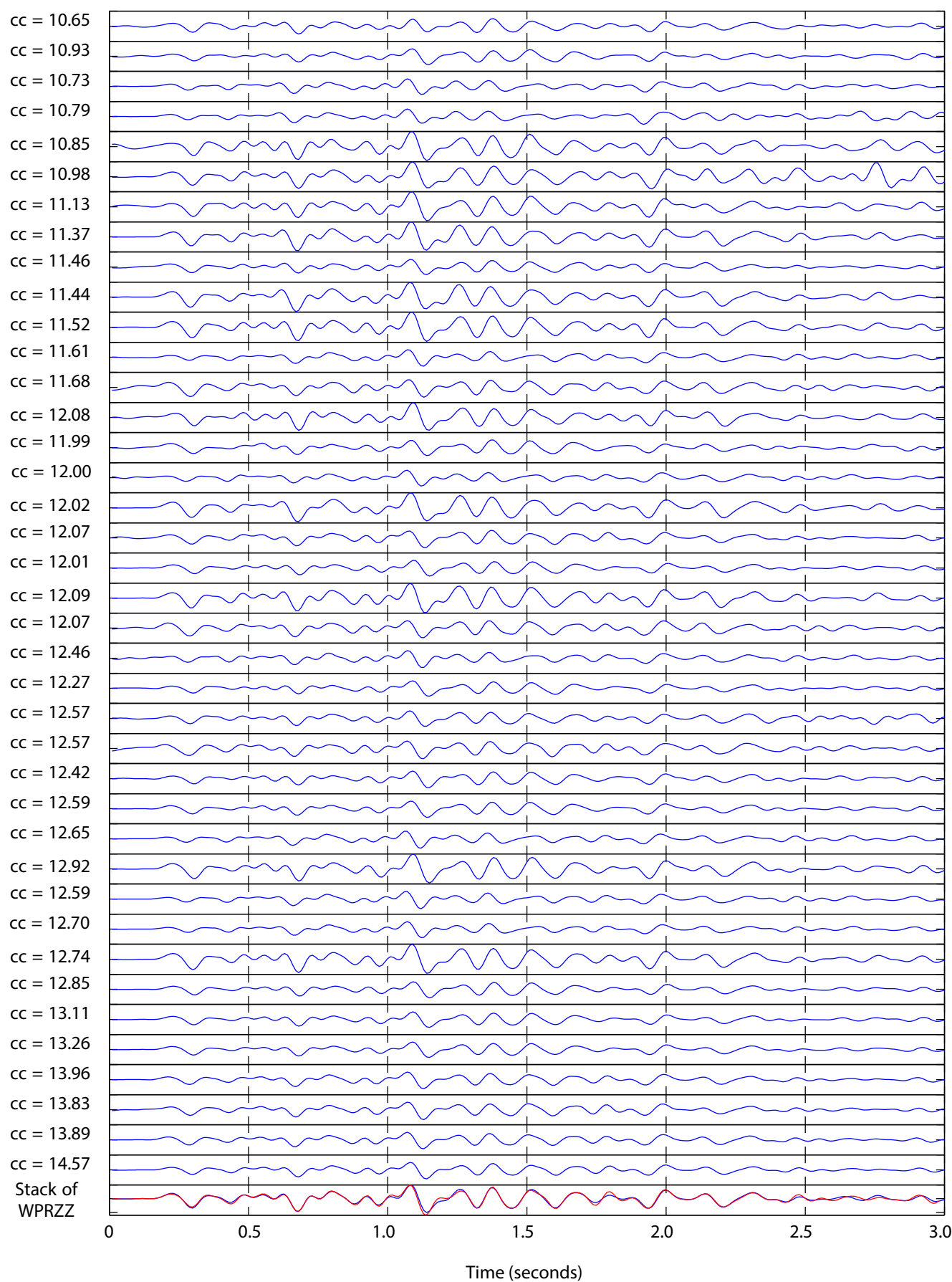

Time (seconds)

Figure 4.6: Individual detections and full detection stack for the highest $6 \%$ (39 detections) of correlations by template from 16 June 2012 recorded on the vertical component of station WPRZ. Detection waveforms are in blue with the network correlation sum to the left of each waveform. In the last line, the linear stacked waveform (blue) is overlain by the template waveform (red). 
Stack of event 20120617_211506.44 NS08E

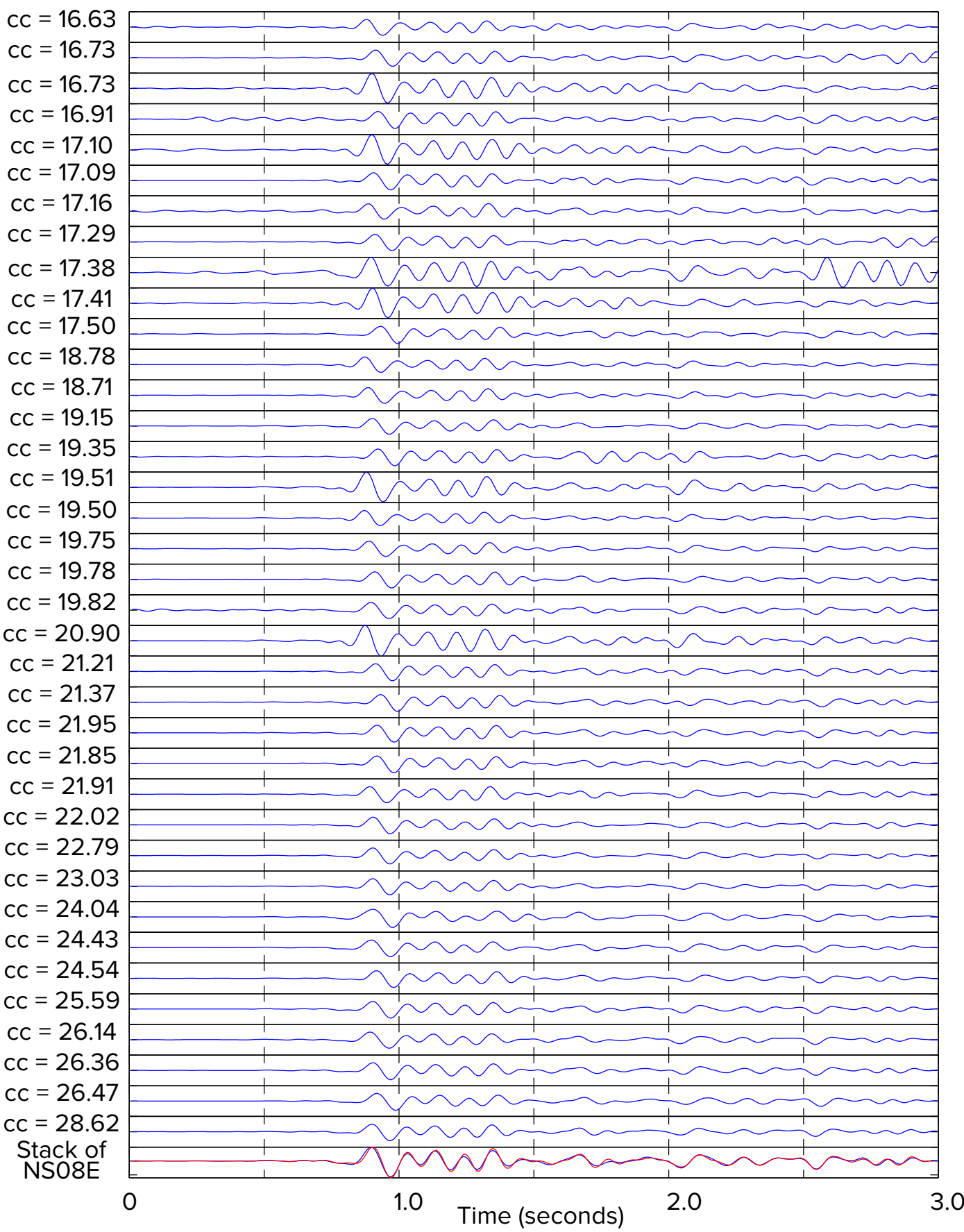

Figure 4.7: Individual detections and full detection stack for the highest $6 \%$ (37 detections) of correlations by template from 17 June 2012 recorded on the eastern component of station NS08. Detection waveforms are in blue with the network correlation sum to the left of each waveform. In the last line, the linear stacked waveform (blue) is overlain by the template waveform (red). 
the modal distribution of events in time between the clusters. The templates in multiplets 1, 3, and 4 are detecting events primarily during June 15-27 and July 410, where as templates in multiplets 2, 5, 8, and 10 detect mostly events July 17-29, leaving only sporadic temporal overlap (Figure 4.8). To avoid the sporadic nature of template detection outside of the primary detection time interval, we perform the time comparison only between detections that were detected by templates in the similar geographic region of the field.

We also observe that smaller magnitude templates detect more events (Figure 4.9). For template magnitudes less than 1.5, we observe multiple templates of a similar magnitude with sets of detections of varying sizes. For example, a template event of magnitude 0.95 (index 27) detected 295 events, whereas a different template event of magnitude 0.96 (index 29) detected 461 events. This type of occurrence diminishes at template events above magnitude 1.5 (Figure 4.9). Although Figure 4.9 does not show a clear linear relationship, we do observe a general inverse proportionality between template magnitude and number of detections. We note that there is a sparse occurrence of template event magnitude greater than 1.75.

\subsection{Double-difference microearthquake locations}

The double-difference method is very sensitive to initial hypocenter locations and program parameters (Waldhauser and Ellsworth, 2000). We attempt to compute initial locations using a 1-dimensional velocity model (Sherburn and Bourguignon, 2013) and the Seisan Hypocenter utility (Figure 3.12, Table 3.2) (Havskov and Ottemoller, 1999). The utility uses a multistation approach to minimize residuals at multiple stations for a single event while walking the hypocenter from an initial location. This gives us a hypocentral cluster approximately $0.5-1.0 \mathrm{~km}$ from the original locations of the template events and not near the injection wells. We were not able to resolve this difference by systematically changing the HypoDD parameters. In other words, the system of equations that HypoDD uses to calculate the double difference locations is too dependent on the initial locations, and using these unexpected initial locations computed in Seisan results in unsuitable subsequent relocations. The original template locations are constrained enough so that we know the true locations are significantly different than those we compute with Seisan. Although we did not end up using initial locations generated from 


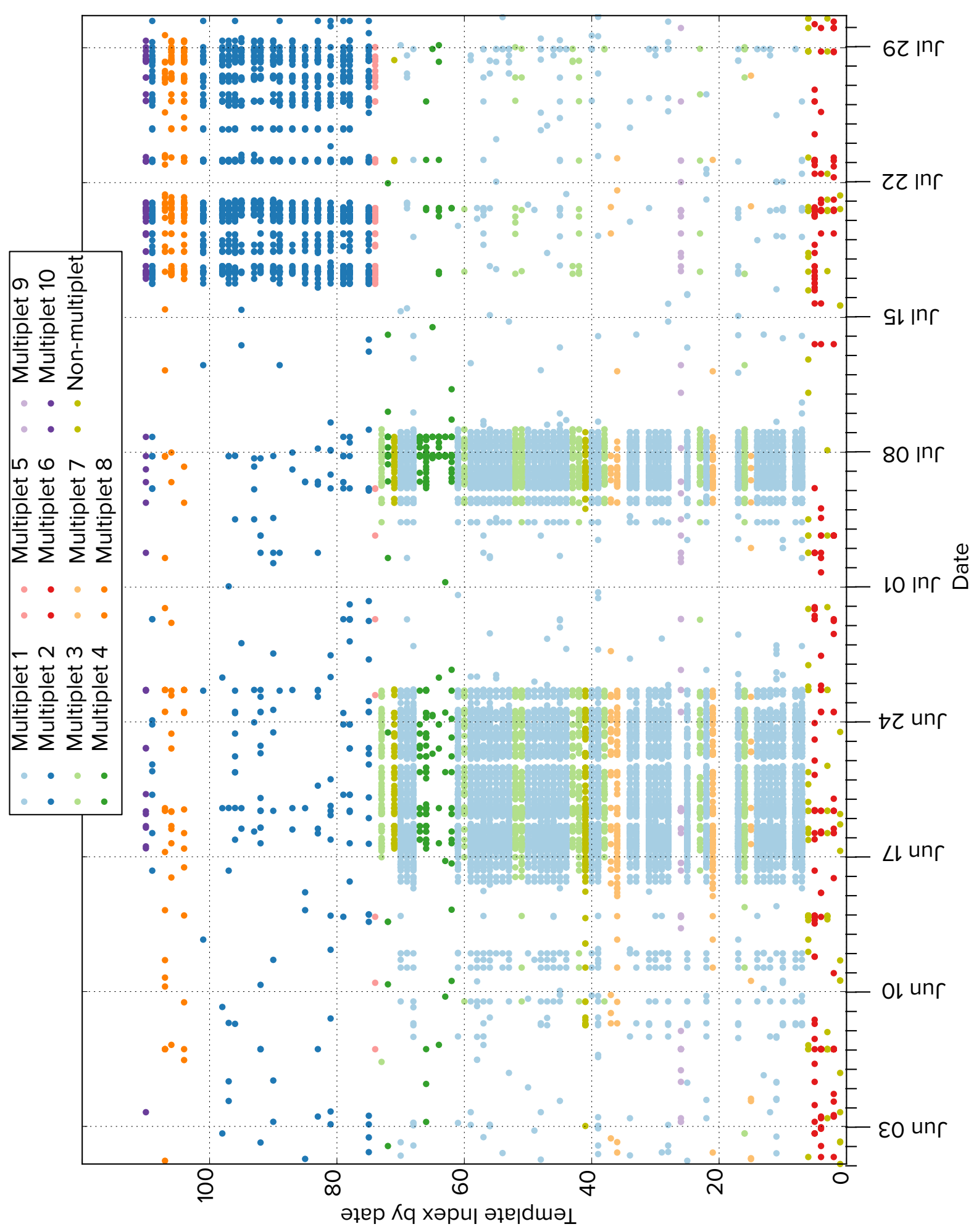

FiguRE 4.8: Timing of each detection from all 110 templates colored by template multiplet. Each point represents a detection for the template in that particular row. Templates are ordered by date, ascending upwards. See Table A for the raw numbers of detections for each template. 


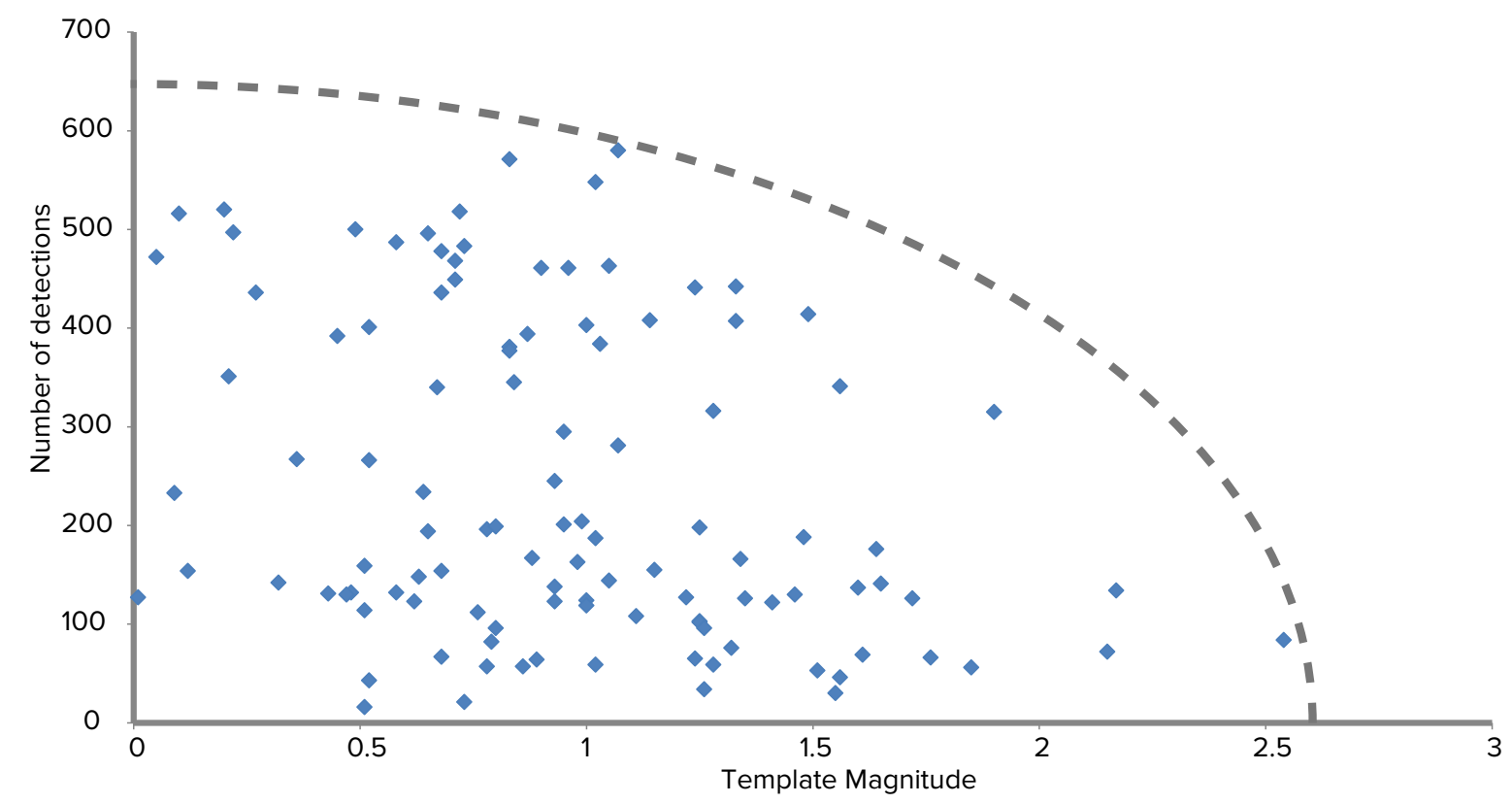

Figure 4.9: Scatter plot of total number of detections versus template magnitude. Each point corresponds to the number of events a template with the corresponding magnitude detected. Although there is not a precise trend of the data, there is a general inverse relationship between template magnitude and number of detections. The grey arc illustrates this general trend.

Seisan, the aforementioned calculation is described to provide full details of this project's methods.

To cope with this challenge, we use the original template location as the initial location of a detection before applying the double-difference relocation method. In this way, we use the robust template locations to our advantage and we are able to calculate relocations that agree with the original template locations.

Double difference relocation results in hypocentral locations that are clearly defined by three main spatial clusters in the field (Figure 4.10). The northernmost cluster sits near well NM8. The 522 event cluster sits at approximately $2.1 \mathrm{~km}$ below sea level. Earthquakes extend $2.5 \mathrm{~km}$ from NM8 (Figure 4.10 and 4.11). Events spread out more readily from NM8 to the south, rather than the north, with a linear feature visible on the N-S cross section of Figure 4.12. A possible explanation for this is that the fault north of NM08 may act as a boundary to fluid, pressure, and temperature movement. Seismicity near NM8 expresses itself bimodally in time, where there are two stages of seismicity, the first of which is from 15 June (time 2) - 25 June (time 3), and the second is from the 4 July (time 5)- 8 July (time 6) (times are denoted on Figure 4.13). 


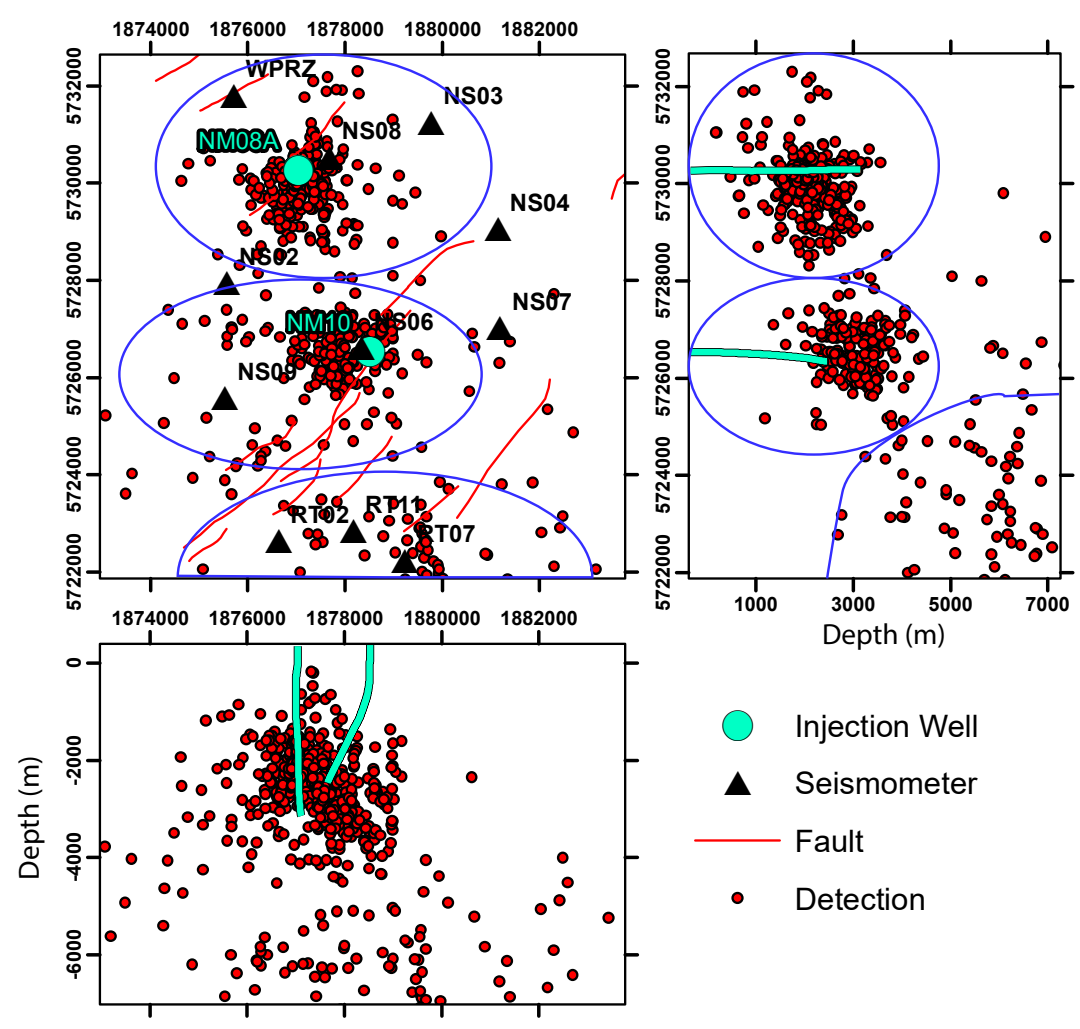

Figure 4.10: Map of detections by double-difference relocation (red) with the seismic network and injection wells NM8 (north) and NM10 (south). Spatial clusters are outlined in blue.

The central area of seismicity contains 188 events and is located slightly west of the southern Ngatamariki injection well, NM10 (Figure 4.10). Seismicity near NM10 is less dense than the cluster near NM8. It's centroid sits near the bottom of the NM10 borehole at approximately $2700 \mathrm{mbsl}$. The cluster extends about $3 \mathrm{~km}$ to the east and is about $2 \mathrm{~km}$ wide in the north-south direction (Figure 4.14). Temporally, seismicty near NM10 is constrained to the last 17 days of July, starting on 14 July (time 2) and continuing until the end of the month (Figure 4.15).

Further south is an area of seismicity outside the Ngatamariki geothermal field (Figure 4.10). This is a poorly clustered group of 152 detections and is the least dense of the three areas of seismicity. This zone of seismicity is deeper than the other two clusters, centered at approximately $6 \mathrm{~km}$ below sea level. It is difficult to define where the cluster stops due to its large, but sparse coverage. In the eastwest direction, the seismicity south of the field extends about $8 \mathrm{~km}$ and about $4 \mathrm{~km}$ in the north south direction (Figure 4.11 and 4.16). In fact, this cluster is mostly located within the Rotokawa geothermal field, not the Ngatamariki geothermal 


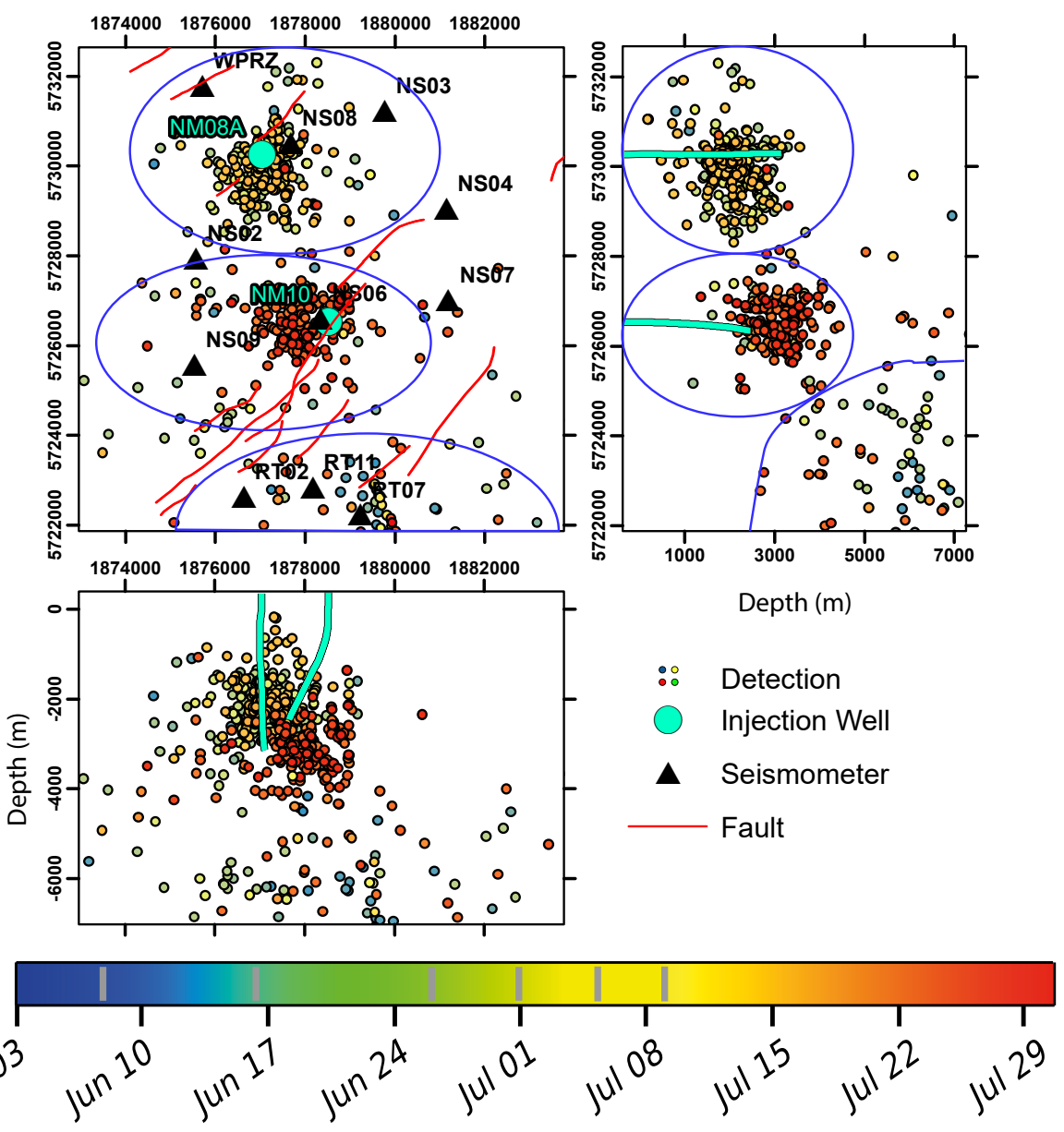

Figure 4.11: Map of relocated detections colored by day of occurrence. The seismic network and injection wells NM8 (north) and NM10 (south) are displayed for reference. Spatial clusters are outlined in blue.

field (Figure 1.3). The cluster's events occur well distributed in time across the two months (Figure 4.17). There are two days, namely 7 June and 19 July during which seismicity rate experiences a small increase of over 20 events per day.

In the following chapter, we will discuss how these clusters of seismicity correlate to geothermal injection and other anthropogenic resource exploitations.

\subsubsection{Uncertainty in earthquake location}

There are two main sources of error when using a double-difference relocation method: (1) errors in initial location and (2) relative relocation error. Here we will discuss how we quantify these errors and how the errors impact the location results. 

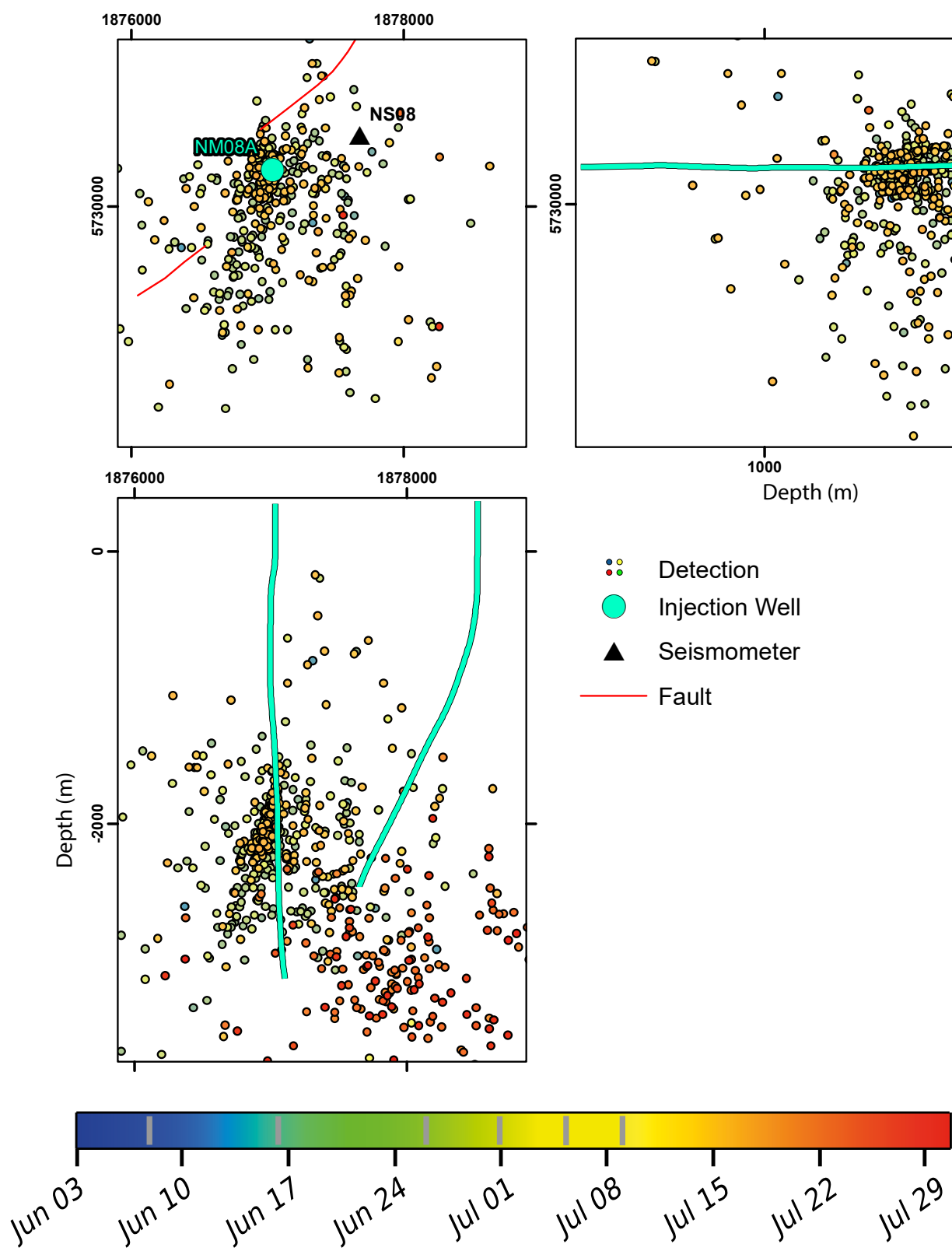

FiguRE 4.12: Enlarged map of detections clustered near NM8. The seismic network and injection well NM8 are displayed for reference. The color of an events corresponds to the day it occurred. 


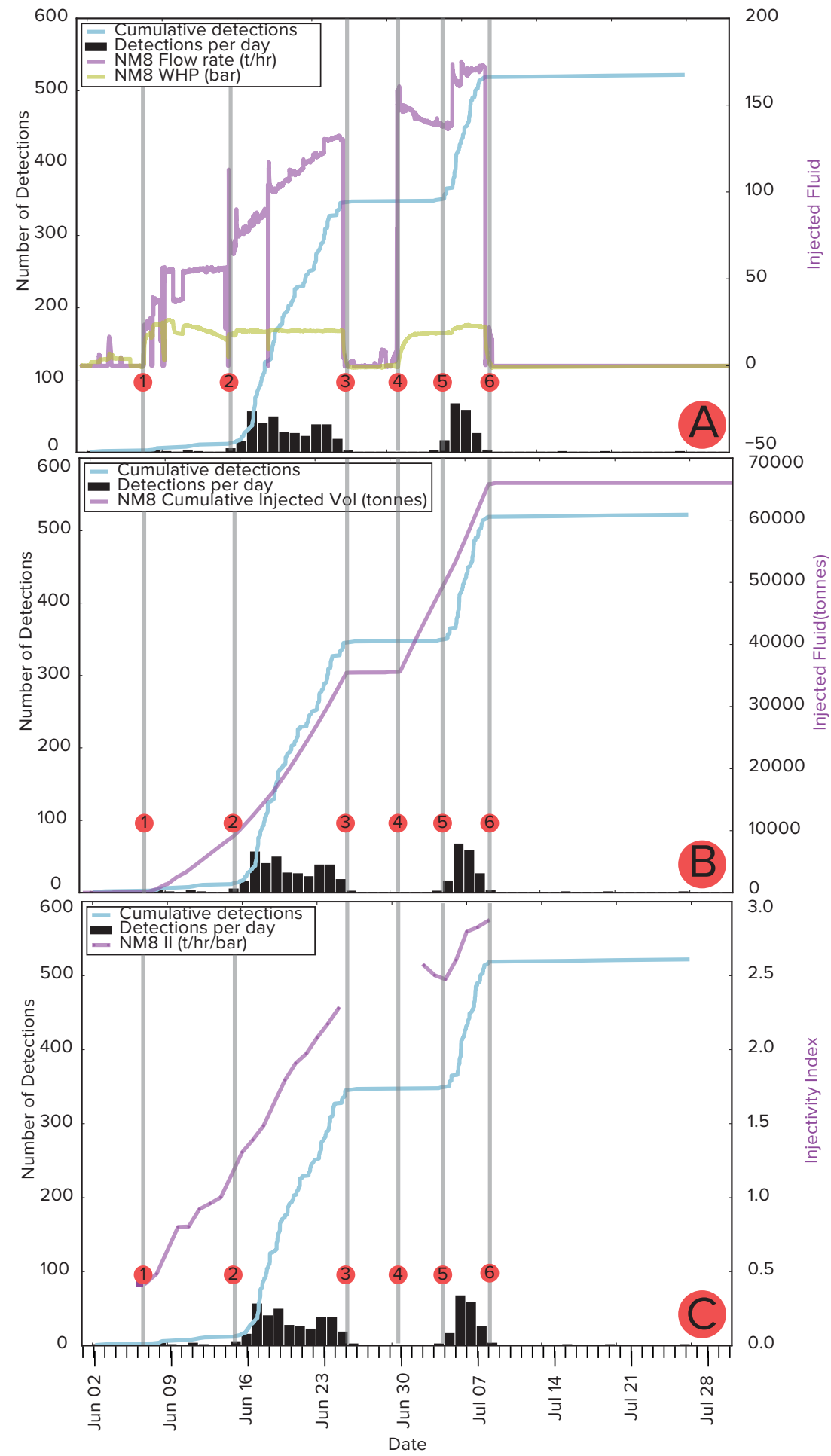

Figure 4.13: Plot of detections (cumulative and per day) from detections in the northern cluster. Here we compare to NM8 (A) injection flow rate and well head pressure, (B) cumulative injected volume, and (C) Injectivity Index (II). Times of interest are numbered: (1) start of injection, (2) increase in seismicity rate, (3) halt of injection and seismicity, (4) restart of injection, (5) restart of seismicity, and (6) halt of injection and seismicity. 


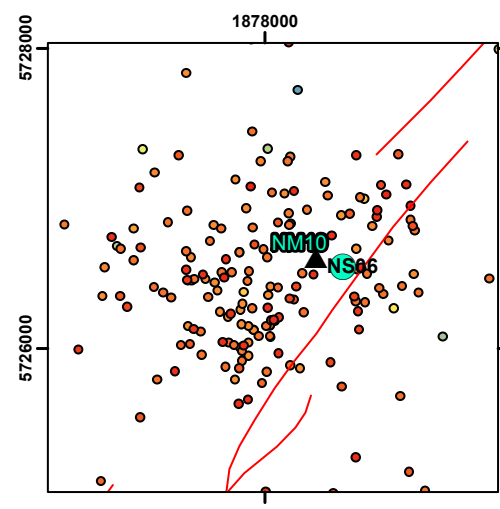

1878000

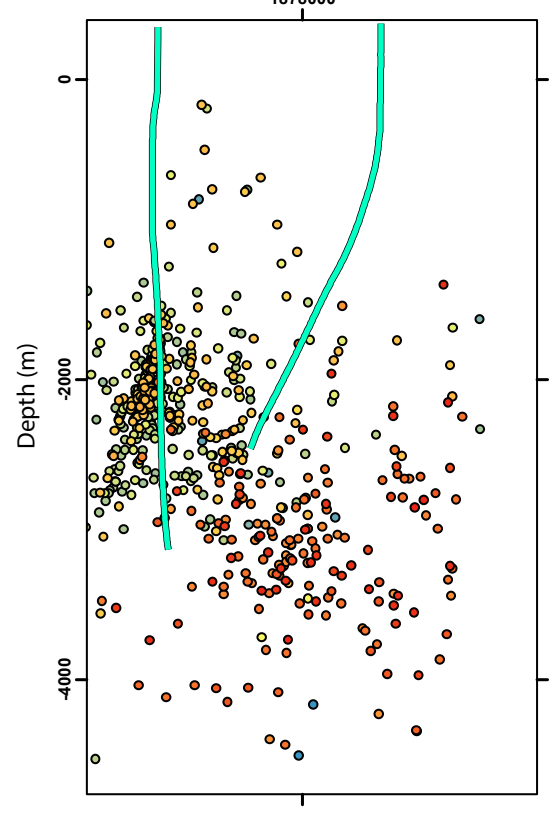

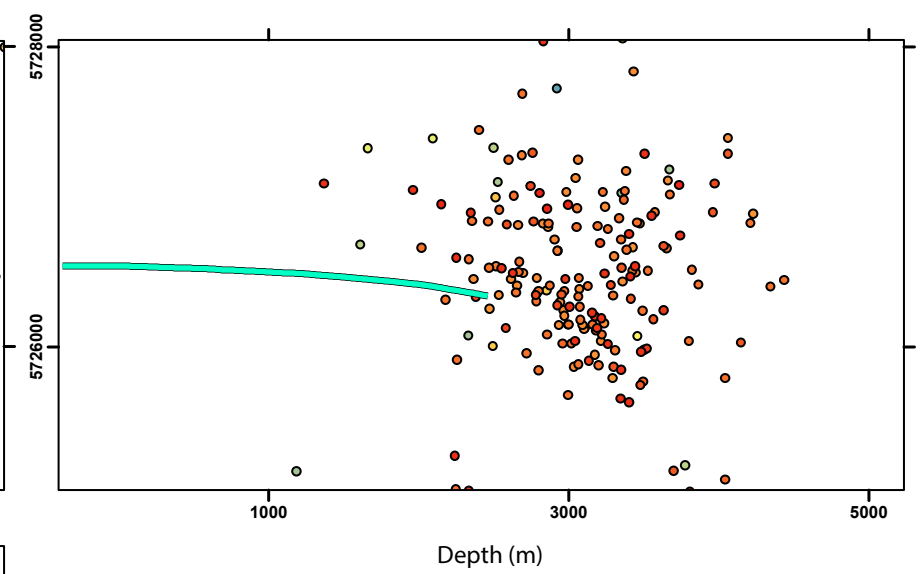

$\therefore$ Detection

Injection Well

- Seismometer Fault

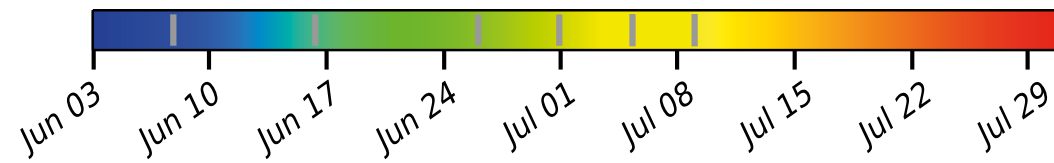

Figure 4.14: Enlarged map of detections clustered near NM10. The seismic network and injection well NM10 are displayed for reference. The color of an events corresponds to the day it occurred.

As described in Section 4.3, determining reliable initial locations was a major challenge of this project, which we solved by using letting the initial location of a detection be that of it's detecting template. We therefore use the original errors on the template locations as errors on the initial detection locations. According to Sherburn and Bourguignon (2013), the accuracy of template event locations is a few 100 meters in map position and as much as 700 meters in depth.

We also consider the errors that result from the double-difference relocation. HypoDD has two ways of solving the system of equations to determine relocations: 


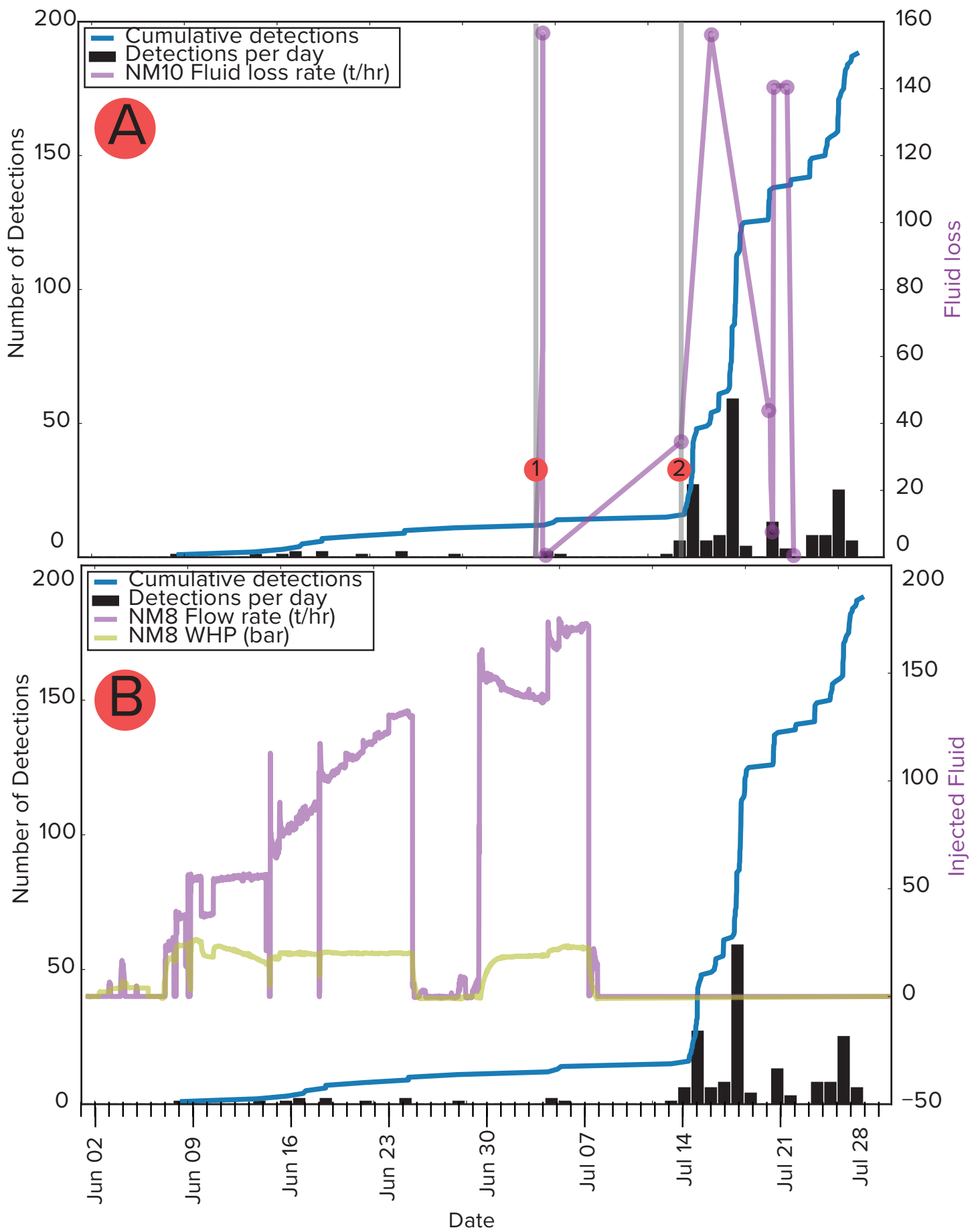

Figure 4.15: Plot of detections (cumulative and per day) from detections clustered near NM10. Here we compare to (A) NM10 drilling fluid loss and (B) NM8 injection flow rate and well head pressure. The reason we compare NM8 flow rate and WHP to seismicity near NM10 is to show that there is not correlation between the two. Actual data from the NM 10 fluid loss rate data set was spars and only exists at changes in slope of the graph. Times of interest are numbered: (1) first instance of drilling fluid loss from NM10 and (2) increase in seismicity rate. 


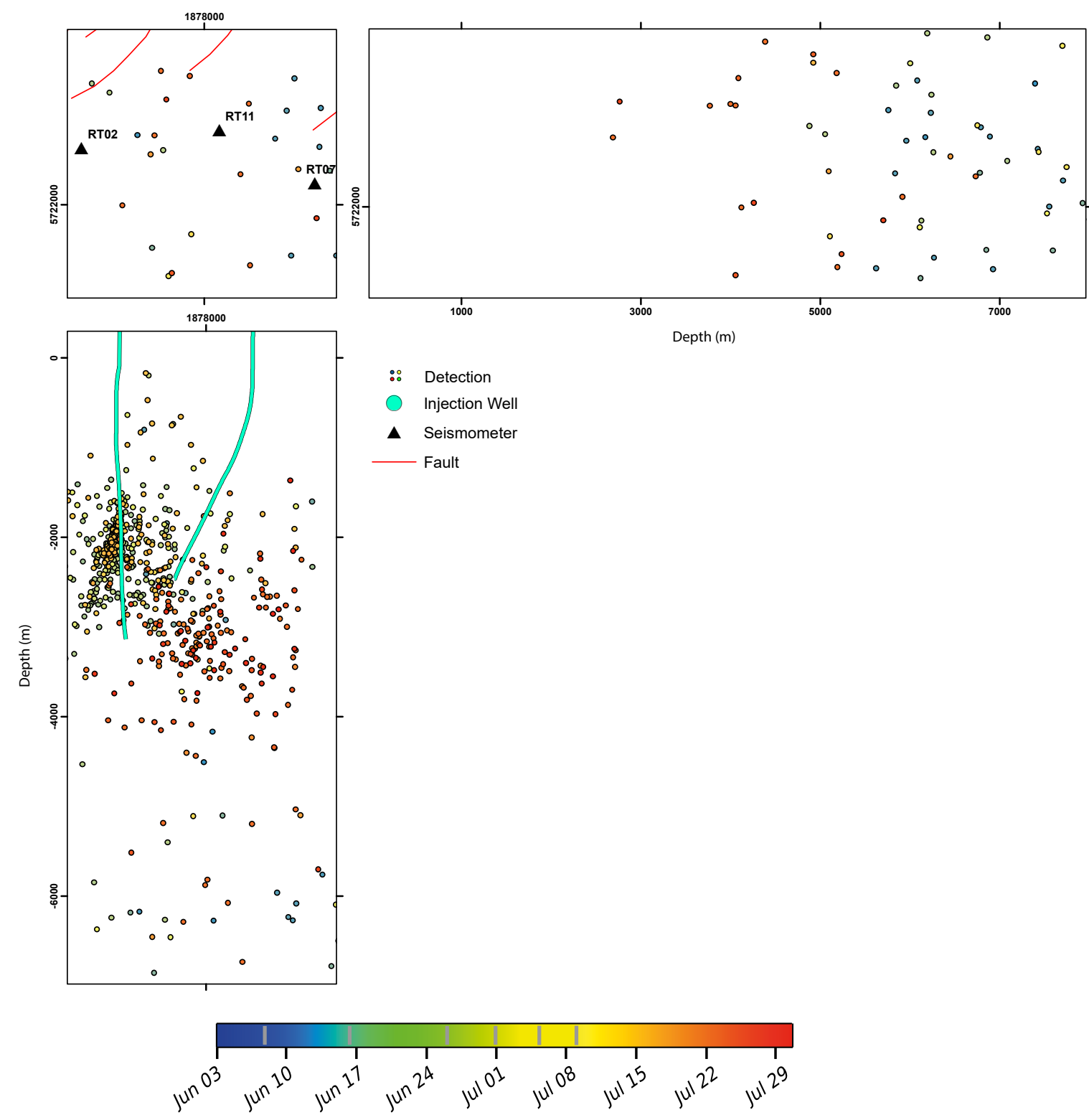

FigURE 4.16: Enlarged map of detections located south of the Ngatamariki geothermal field. The seismic network is illustrated in black. The color of an events corresponds to the day it occurred.

singular value decomposition (SVD) and least-squares (LSQR)(Section 3.8) (Waldhauser and Ellsworth, 2000). The SVD method gives reliable errors, but can only be used for systems of approximately 100 events or less (as noted by Waldhauser and Ellsworth (2000)). Since we cannot obtain useful errors when processing all of the detections at once, we process a subset of the detections to constrain the double-difference errors. We relocate 132 events near NM8 using the SVD method to obtain average errors in three directions (Table 4.1).

We can also inspect the residuals between calculated and observed differential 


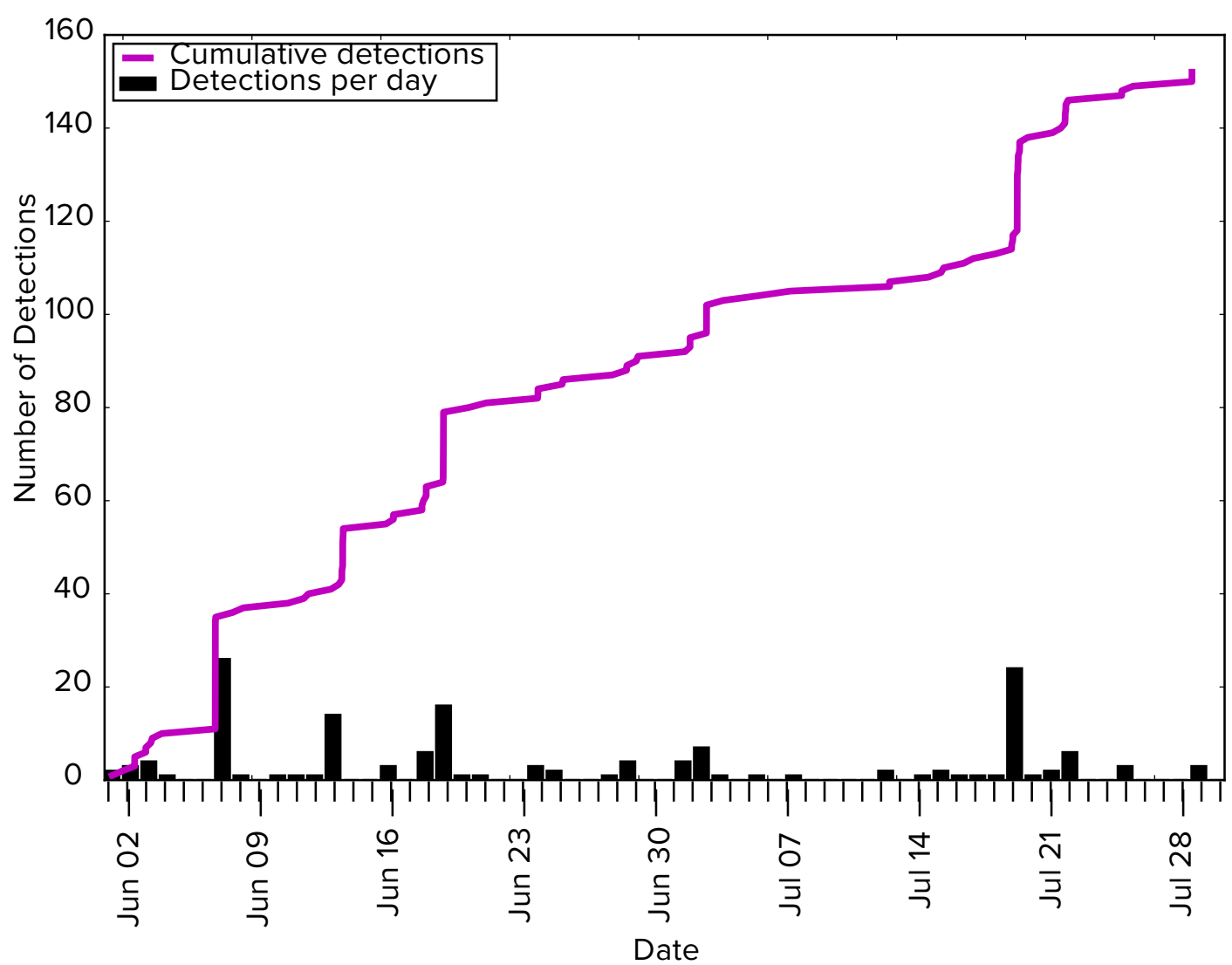

Figure 4.17: Plot of detections (cumulative and per day) from detections in the seismicity cluster south of the Ngatamariki geothermal field.

TABLE 4.1: Double-difference location errors

\begin{tabular}{cc}
\hline Direction & Average error $(\mathrm{m})$ \\
\hline east-west & 9.8 \\
north-south & 12.4 \\
depth & 30.3 \\
\hline
\end{tabular}

travel times. Figure 4.18 illustrates the residuals for each station used to perform the double-difference relocation of the 800 events. The residuals for all stations do not exceed $2.0 \mathrm{~ms}$. Certain stations such as NS04 and NS06 tended to perform poorly in that many measurements were discarded. We can come to this conclusion by noting that there are orders of magnitude fewer observations of differential travel times on these two stations. 

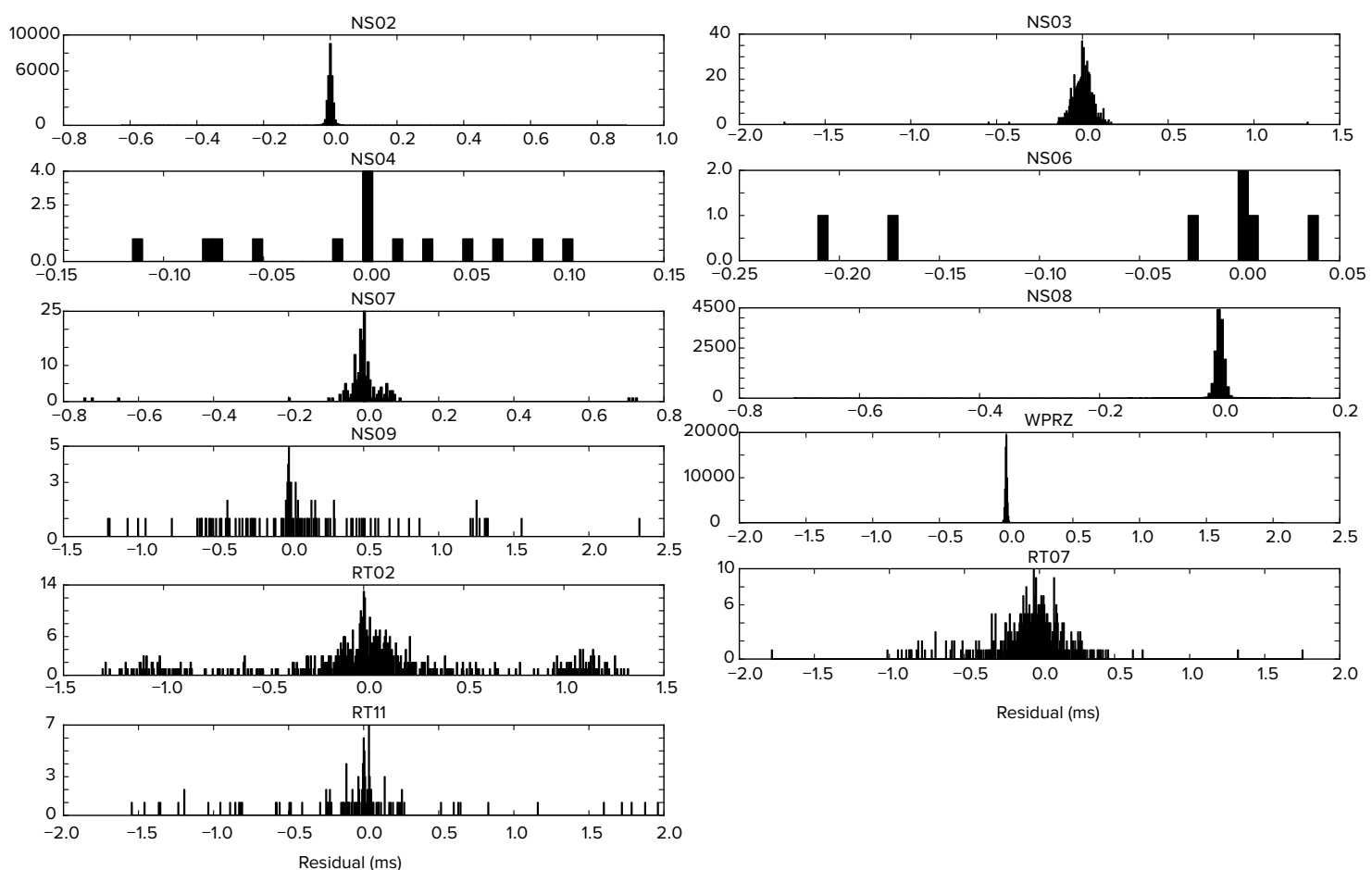

Residual (ms)

FiguRE 4.18: Histograms of remaining residuals between the calculated and observed travel times between pairs of events for each station. The horizontal axis gives the residual (ms) and the height of the column gives the frequency of that residual.

\subsection{Singular value decomposition magnitudes}

We use an singular value decomposition method developed by Rubinstein and Ellsworth (2010) to calculate local magnitudes $\left(\mathrm{M}_{l}\right.$, Secion 3.7 for 341 of the detections from templates located near NM8 (Figure 3.1). The SVD method allows only those detections with exceptionally high correlation to have a magnitude calculated (Rubinstein and Ellsworth, 2010). Magnitudes range from $-0.15 M_{l}$ on June 17th to $1.66 M_{l}$ on June 23rd (Figure 4.19). We see that larger magnitude events $\left(>1 M_{l}\right)$ and smaller magnitude events $\left(<0.5 M_{L}\right)$ occur throughout the stimulation period. Figure 4.20 illustrates that we have higher numbers of events with smaller magnitudes. We plot the frequency-magnitude distribution for the set of detections using the method of Wesnousky (1994) to define the GutenbergRichter relationship of our catalogue, as discussed in Section 3.7.1(Figure 4.21). We calculate a b-value of $b=0.989$ using a completeness magnitude of $M_{c}=0.25$. Figure 4.21 shows how this b-value fits the frequency-magnitude. We observe that the b-value is a better fit for smaller magnitudes. This is purposeful so that the 


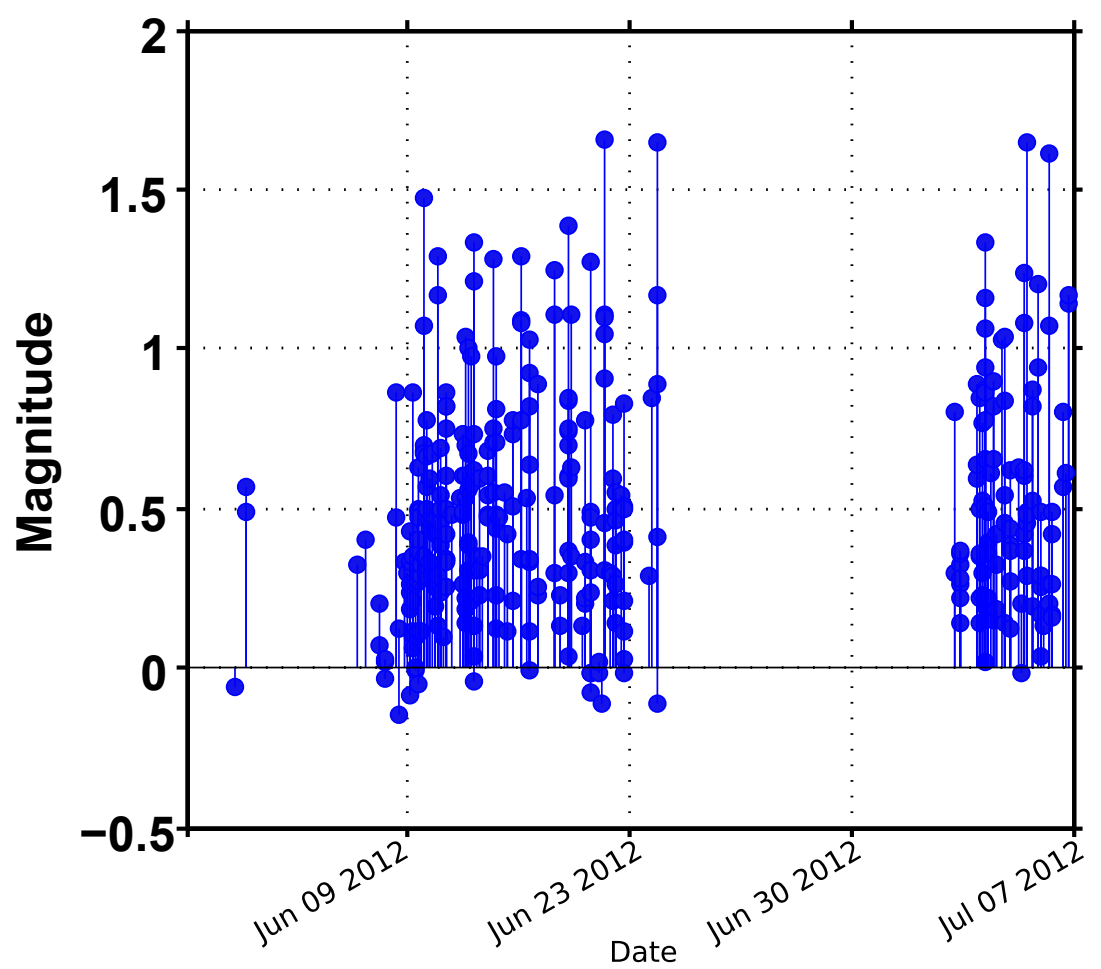

Figure 4.19: Plot of local magnitude over time. Each point represents a detection in the seismic cluster near NM8. The height from the time-axis represents the event's magnitude.

larger magnitudes, for which we did not detect many, do not skew the computation to find a b-value and inflate it unnecessarily.

\subsection{Diffusion of seismicity}

We compare the seismicity distribution with time by calculating the distance of detections from injection well NM8 since the start of injection (Figure 4.22). For each day we compute a box and whisker plot for the distribution of seismicity and overlay this on top of the individual earthquakes. This shows the range of seismicty by day along with the median and upper and lower quartiles of seismicity with distance. Additionally, Figure 4.22 shows the diffusion envelopes of hydraulic pore fluid pressure front traveling from NM8 radially outward through the reservoir assuming hydraulic diffusivity of $D=0.16 \mathrm{~m}^{2} / \mathrm{s}, D=0.05 \mathrm{~m}^{2} / \mathrm{s}$, and $D=0.01 \mathrm{~m}^{2} / \mathrm{s}$. In the following chapter, we compare the pore fluid pressure front with the time evolution of seismicity with distance to give insight into the possible mechanisms of microseismicity near NM8. 


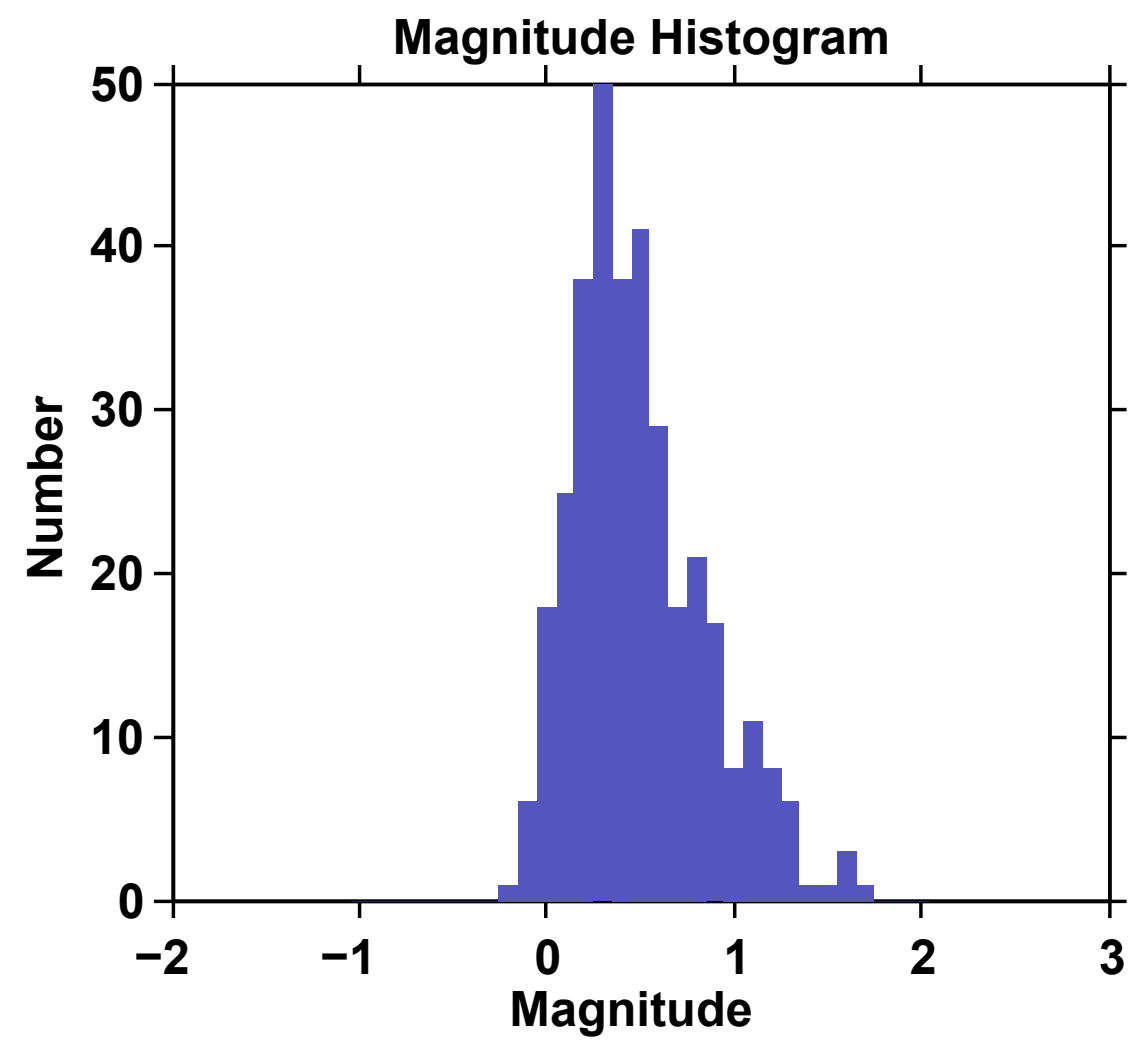

Figure 4.20: Local magnitude histogram where each column represents the number of occurrences of a certain magnitude range. 


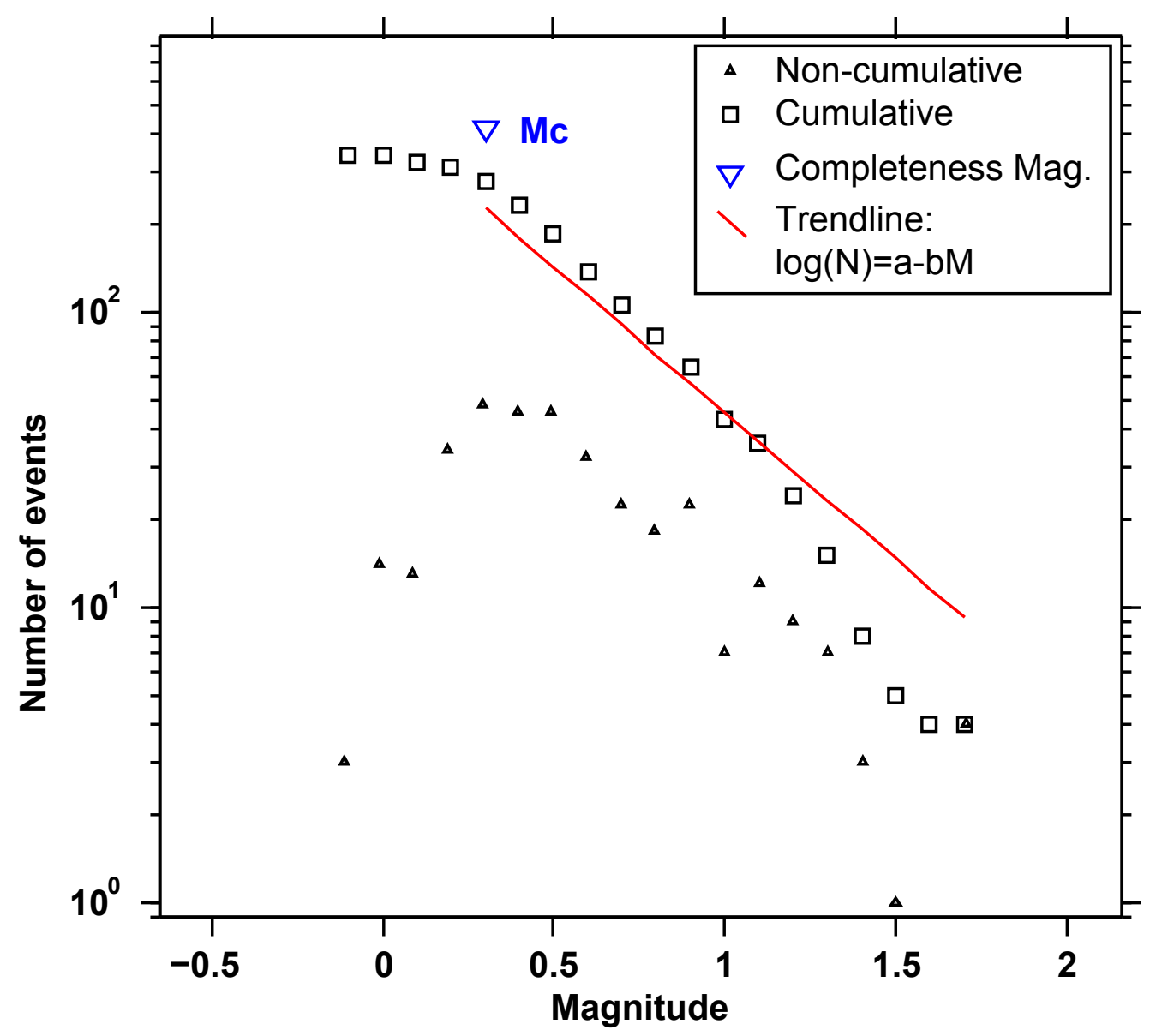

Maximum Likelihood Solution

b-value $=0.989+/-0.04$, a value $=2.65$

Magnitude of Completeness $=0.25$

Figure 4.21: Gutenberg-Richter plot for detections with local magnitudes. We calculate a completeness magnitude of 0.25 and a b-value of approximately 0.989 


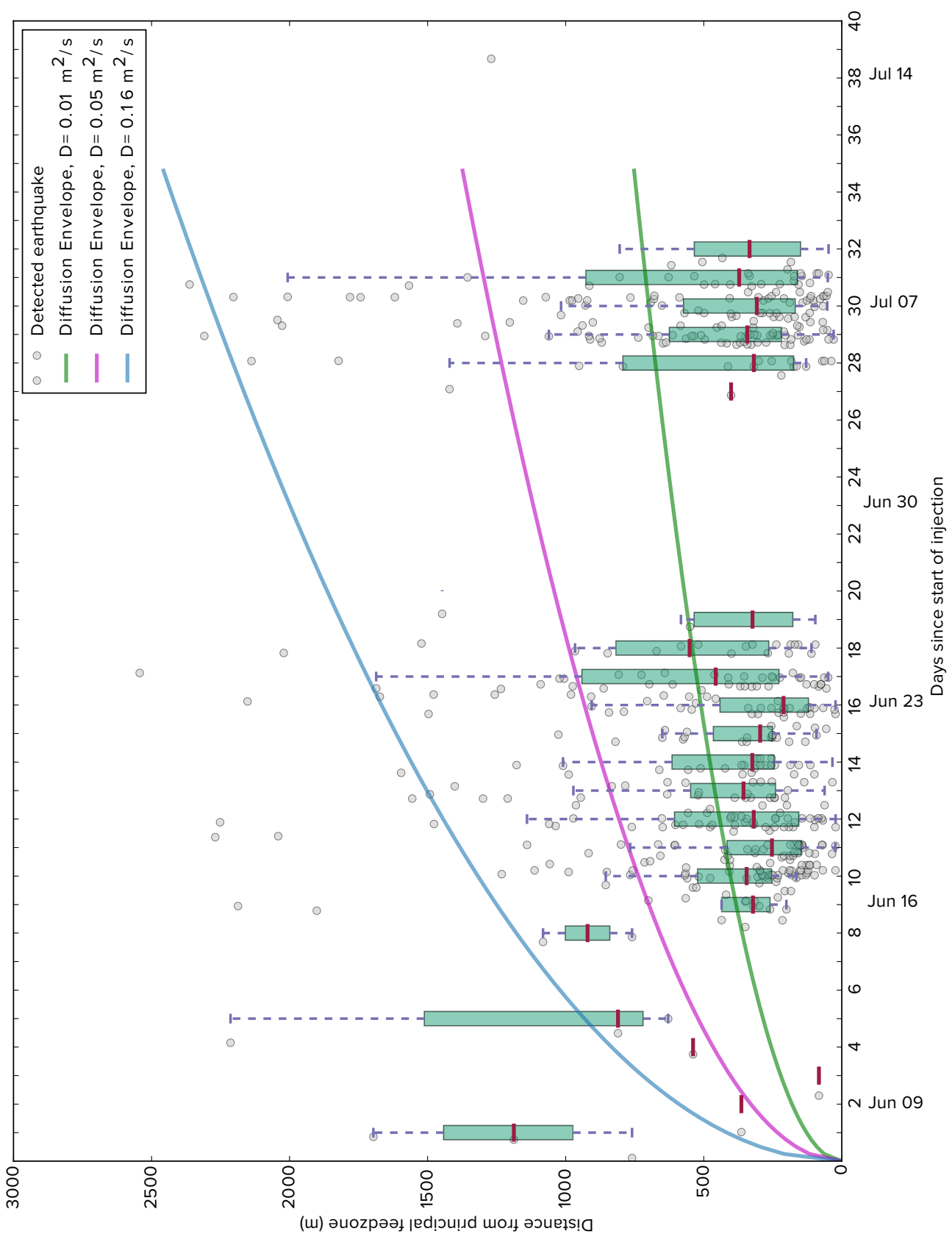

FiguRE 4.22: Scatter plot of radial distance of microseismicity from injection well NM8 during stimulation. Earthquake detections near NM8 (grey circles) are plotted by their distance from the location of fluid injection at the origin time of the event. A box and whisker plot for each day's detections are overlain in the detections themselves. For each box and whisker plot, we display the daily median distance (red tick mark) and the first and third quartiles (edges of the green box). The first quartile is placed at the point for which $75 \%$ of the data lies below it Similarly, $75 \%$ of the data lies above the third quartile. The dashed lines extend to the maximum and minimum values for that day, excluding outliers. An outlier is determined based on the tightness of the data, i.e. the first quartile-third quartile difference. We display three curves each representing the radius of pore fluid pressure being injected from NM8 and radiates outward 


\section{Chapter 5}

\section{Discussion}

\subsection{Induced seismicity}

Determining whether seismicity is induced requires a proximity to a plausible mechanism of seismicity in both space and time (outlined in Section 2.3). We present arguments based on the results presented in Chapter 4 as to whether or not the three clusters of seismicity are induced.

\subsubsection{Seismicity near NM8}

The northern most group of seismicity clusters around well NM8. This spatial correlation to a source of injection, suggests that the seismicity could be induced (Figure 4.10). Spatially, precise double difference locations show seismicity as a cloud around NM8. Temporal seismicity trends provide compelling evidence that this cluster is induced via injection. Figure 4.13, shows a strong correlation between seismicity and injection. Figure 4.13 illustrates fluid injection in three ways: (A) injection flow rate and well head pressure (WHP), (B) cumulative injected volume, and (C) Injectivity Index, or II. Injection flow rate and well head pressure are used to calculate II (see Section 2.1). We will refer to the times highlighted on Figure 4.13 as T1, T2, T3, etc. to be clear about what point on the curves we are referencing.

We see an eight day lag between injection flow rate commencing on 7 June, T1, and a spike in seismicity rate on 15 June, T2 (Figure 4.13). We also see an 
increase in cumulative injected volume at time T1 and although II measurements begin at time T1, data are increasing with time. On June 25th, T3, we observe a halt in injection, as injection flow rate and WHP fall to zero. Simultaneously, we observe a sharp decline in seismicity rate, without any lag, at T3. There is void of data between T3 and shortly after T4 on the II curve, due to a lack of measurements. On 30 June, T4, fluid injection restarts with an increase in flow rate to approximately $150 \mathrm{t} / \mathrm{hr}$. We observe a lag of 4 days before seismicity rate increases on 5 July, T5. On 8 July, T6, we observe the same behaviour as the earlier halt in injection: an immediate decrease in seismicity rate.

Injectivity Index increases with time throughout the stimulation period, except for a two days before T5 (Figure 4.13) (Clearwater et al., 2015). The overall increase in II is attributed to cooling of the rock near the well, which thermally expands fractures and improves permeability (Clearwater et al., 2015, Grant et al., 2013). As II increases, the permeability of the reservoir increases (Clearwater et al., 2015, Grant et al., 2013). We see that seismicity rate increases along with II (Figure 4.13). Clearwater et al. (2015) attribute the decrease in II before T5 as a transient phase of injectivity, and not reflective of real reservoir permeability changes.

Shapiro and Dinske (2009) derive numerically that in the case of constant fluid injection rate (different from II), that the rate of seismicity should be proportional to the injection rate. The proof of this is beyond the scope of this project, but the governing equation in essence gives the probability of an event occurring to be proportional to the time and rate of fluid injection. Figure 4.13 illustrates the average flow rate by integrating the instantaneous rate to show cumulative injected volume. In the first period of stimulation $(\mathrm{T} 1-\mathrm{T} 3)$ we see the injection rate increase with time, as the cumulative injected volume curve has positive concavity. The later part of the stimulation (T4 - T6) exhibits a more constant injection rate and seismicity rate (Shapiro and Dinske, 2009, Shapiro, 2015).

In summary, NM8 fluid injection during June and July 2012 is highly correlated with seismicity (Figure 4.13). Not only is seismicity correlated in time, but we locate the seismicity as a cluster centered around the NM8 borehole. The spatiotemporal correlation of injection and seismicity is sufficient evidence to classify this northern cluster as induced via cold fluid injection during NM8 stimulation tests. The specific mechanism (pressure versus temperature) will be discussed in Section 5.2. 


\subsubsection{Seismicity near NM10}

The second area of seismicity is located just to the west of injection well NM10. This seismicity is not temporally correlated with the NM8 stimulation (Figure 4.15B). We do not see a spatial correlation with seismicity near NM10 and injection from NM8. In other words, there is a significant spatial gap in seismicity between the two clusters, indicating that fluid flow from NM8 is not advecting readily to the seismic cluster at NM10. This is supported by hydrological models of Ngatamariki (Boseley et al., 2010) and recent tracer tests (Buscarlet et al., 2015). Instead, we consider NM10 as a source for fluid input that can induce seismicity. In Section 3.1, we note the timing of NM10 drilling and the fluid losses associated with drilling NM10. Figure 4.15A shows the seismicity compared to drilling fluid losses at NM10. NM10 fluid loss rates are measured much less frequently than the NM8 injection data, making it difficult to attribute specific changes in fluid loss to specific changes in seismicity. Despite this, the relative timing of fluid loss during NM10 drilling and the seismicity coincide well.

This cluster of seismicity is located near the bottom of the well (Figure 4.10). Drilling fluid loss occurred while the borehole was between 1800 and $2800 \mathrm{mbsl}$, which is just above the seismicity cluster's center. Figure 4.14 shows a clear lineation of seismicity on the north-south cross section. The lineation appears to be emanating from the end of the NM10 borehole, and is parallel to the trend line of the adjacent fault. The location of this area within the cluster provides more spatial correlation between NM10 and the seismicity cloud surrounding it. Although these events coincide coincide with the trend of the active fault in the vicinity of NM10, which may imply that a subset of these events are 'natural' events, we believe strongly that based on the temporal relationship to NM10 fluid loss that these events are a majority, if not entirely induced. The spatiotemporal correlation between drilling NM10 and seismicity to the west is sufficient evidence to classify this cluster as being induced by drilling fluid loss.

\subsubsection{Seismicity south of Ngatamariki}

Seismicity in the area south of the Ngatamariki field, near seismic stations RT02, RT07, and RT11 is deeper (6 km) and poorly clustered (Figure 4.10). These events are relatively evenly distributed through time during June and July 2012 (Figure 
4.16). There is no source of fluid, such as an injection or production well, near the seismicity. Furthermore, there is no fluid injection rate or otherwise to compare with the seismicity rate in time. With only access to geothermal exploitation data from Ngatamariki, and not the Rotokawa field to the south, we cannot determine with any certainty whether the deep seismicity south of Ngatamariki is induced. We conclude that that the cluster outside of the field is not induced by either drilling fluid loss from NM10 nor injection from NM8. There is no temporal signature in this cluster to support that these events are induced by either of the aforementioned fluid sources. Additionally, there is no evidence for fluid flow from either of these wells to the southern region of our study area. If fluid was traveling as far south as Rotokawa, we would expect to see a spatial trend of seismicity connecting these three areas. There is no evidence to suggest that seismicity south of Ngtatamariki is induced by a source within Ngatamariki.

\subsection{Mechanisms of induced microseismicity}

Here we discuss a main goal of this project: to use microseismicity near NM8 to determine a mechanism for induced seismicity. We attempt this task considering spatiotemporal variations in microseismicity during a two month period. We propose a mechanism of induced seismicity whereby thermoelastic stresses caused by temperature variations are coupled with diffusion of pore fluid pressure to reduce the normal stress across fractures (Section 2.3.1). The combined thermo-poroelastic stress mechanism has been observed at the Geysers geothermal field, California, United States by Martínez-Garzón et al. (2014) and modelled by Rutqvist et al. (2015). The Geysers field is a high temperature geothermal field in an extensional environment, similar to the Ngatamariki field; with the difference that the Geyesers is a steam dominated reservoir, while Ngatamariki is a two-phase reservoir. Martínez-Garzón et al. (2014) propose that thermoelastic effects and poroelastic effects can both take effect simultaneously, but at different length scales. Below we will describe the argument for both thermoelastic stress or poroelastic stress as a mechanism and then describe why a combination of the two is best suited to describe the induced microseismicity near NM8. 


\subsubsection{Thermoelastic stress}

Recall from section 2.3.1 that the increase of Injectivity Index during stimulation is a result of increased reservoir permeability. Increase in reservoir permeability is widely attributed to temperature differences between the injected fluid and the geothermal reservoir (Clearwater et al., 2015, Grant et al., 2013, Mossop, 2001). The temperature difference between the $20^{\circ} \mathrm{C}$ injectate and the $230^{\circ} \mathrm{C}$ reservoir is enough to cause thermoelastic stress that can induce earthquakes (Bodvarsson, 1972, De Simone et al., 2013).

Sherburn et al. (2015b) and Sewell et al. (2015) both attribute induced microseismicity at the Rotokawa geothermal field to thermoelastic stresses as opposed to pore fluid pressure increase. They deduce this to be the mechanism based on three factors: (1) small difference between the maximum principal stress (vertical) to the minimum principal stress (NW-SE) (Davidson et al., 2012, Townend et al., 2012), (2) the large temperature difference between injected fluid $\left(100^{\circ} \mathrm{C}\right)$ and the reservoir $\left(335^{\circ} \mathrm{C}\right)$, and $(3)$ the changes in pressure in one area of injection have not exceeded 0.1 MPa. There is a similar temperature difference between the reservoir and the injected fluid at Ngatamariki $\left(200^{\circ} \mathrm{C}\right)$. This is enough temperature difference to cause thermoelastic stress perturbations that can induce seismicty (Bodvarsson, 1972, De Simone et al., 2013). We employ a numerical model developed by Bodvarsson (1972) to quantify the potential of a thermoelastic mechanism of seismicity at Ngatamariki. Bodvarsson (1972) models the subsurface temperature around an injection well during injection of cold fluid into a hot, fractured reservoir (Figure 5.1). Considering a point at the edge of or within the permeable fractured zone, then we use the following to estimate the extent of the thermal front:

$$
\begin{array}{r}
T=T_{0} \operatorname{erf}\left(\frac{\pi b r^{2}}{2 \sqrt{\kappa t}}\right), \\
b=2 k / s Q
\end{array}
$$

where $T$ is the temperature of the injected fluid, $r$ is the radius of the front, $T_{0}$ is the temperature of the reservoir, $t$ is the time since injection, $\kappa$ is thermal diffusivity of the rock, $k$ is the thermal conductivity of wet rock, $s$ is the heat capacity of the fluid, and $Q$ is the mass flow rate (Bodvarsson, 1972). 


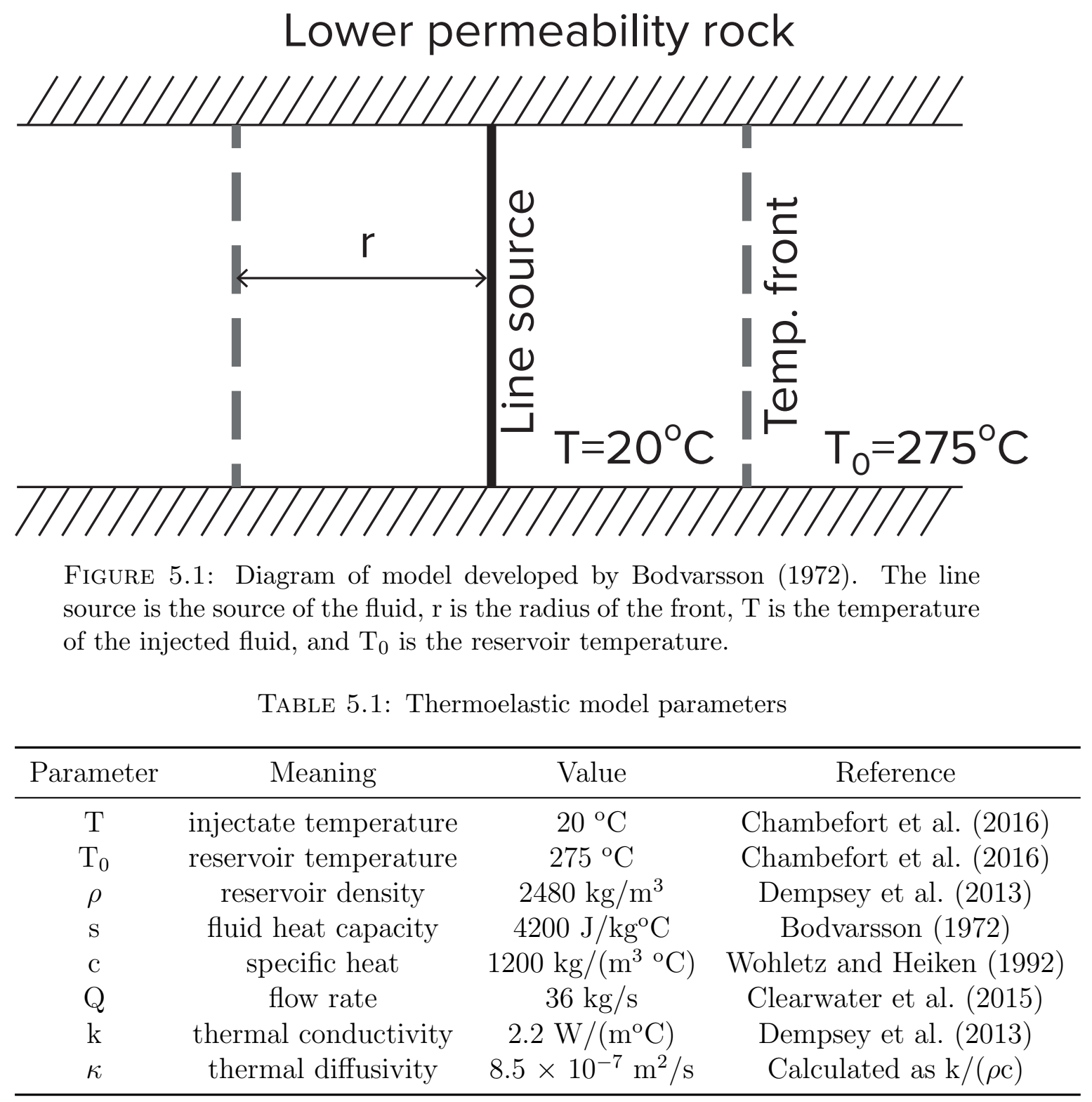

We use the parameters in Table 5.1 to apply the numerical model to calculate how temperature varies with radial distance from the injection source. We calculate the evolution of temperature for a range of time-scales after injection starts. An assumption made by this model is that there is a constant injection flow rate. Since injection into NM8 is not constant (Figure 4.13), we take the flow rate to be the maximum flow rate during the first stage of injection. Assuming a higher flow rate gives us an upper bound on how far a temperature perturbation will have traveled in a given amount of time. Figure 5.2 shows the results of applying the numerical model to the parameters in Table 5.1. We can see that once the first stage of injection ends after approximately 20 days, the temperature decrease of $10^{\circ}$ only reaches $190 \mathrm{~m}$ from the injection well. We observe seismicity within 


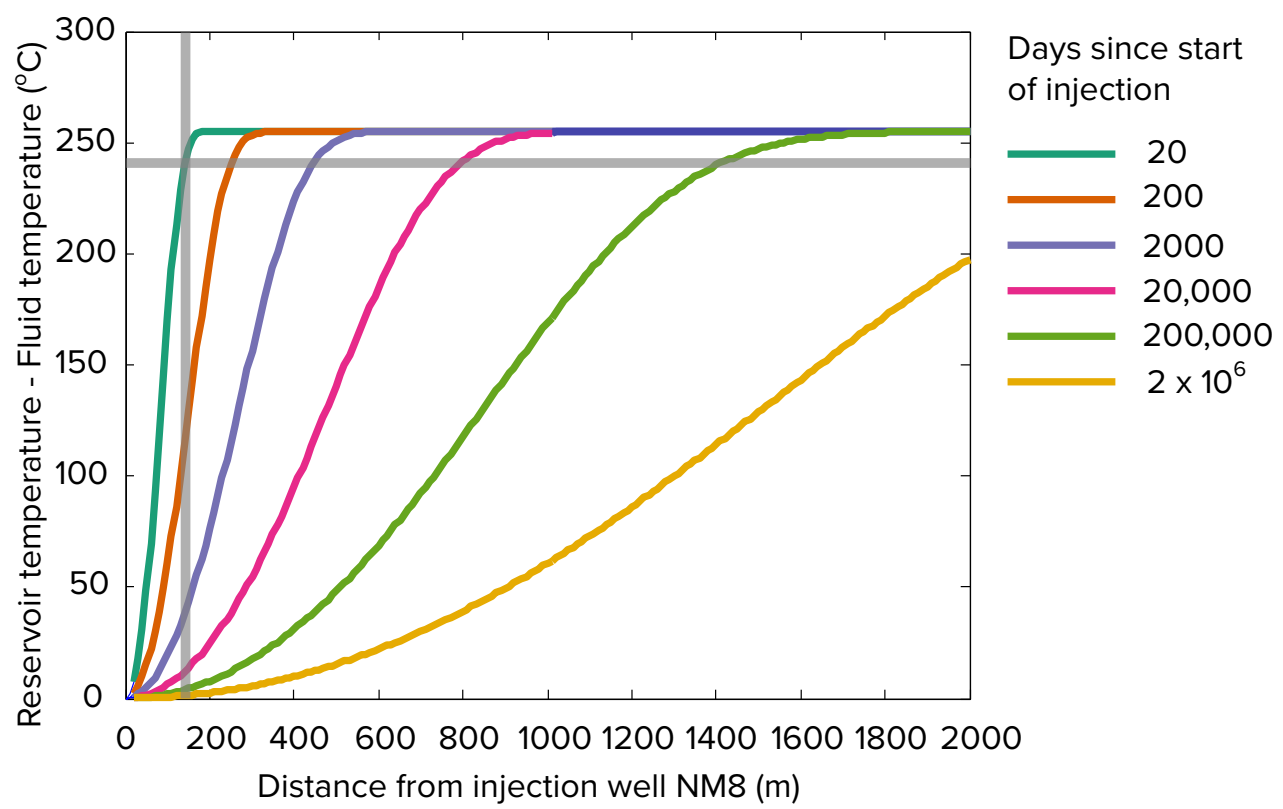

Figure 5.2: Plot of evolution of the radial temperature profile with time assuming the Bodvarsson (1972) thermal model and the parameters in Table 5.1. The vertical axis is measured in reservoir temperature corrected for the temperature of the injected fluid, i.e. we subtract the fluid temperature from the initial reservoir temperature and then assume the fluid being injected is $0^{\circ} \mathrm{C}$. The horizontal gray line is reference for a $10^{\circ} \mathrm{C}$ decrease in reservoir temperature. The vertical gray line illustrates the distance away from injection corresponds to a decrease of $10^{\circ} \mathrm{C}$ approximately 20 days after injection starts.

$190 \mathrm{~m}$ of NM8 (+/- $100 \mathrm{~m}$ (Sherburn and Bourguignon, 2013)) (Figure 4.22). And yet, earthquakes also occur at distances of up to $1500 \mathrm{~m}$ during the first stage of injection at NM8 (while taking into account errors of original location (100 $\mathrm{m}$ ) and earthquake relocation ( $35 \mathrm{~m}$ on average)), much further than the temperature decrease, and thus further than any thermoelastic stress could have occurred. Therefore, we can say that thermal contractions of the reservoir rock due to injection of cold water does induce seismicity near NM8; however, there needs to be another mechanism to induce events greater that $200 \mathrm{~m}$ from the well during the first stage of injection.

\subsubsection{Poroelastic stress}

We describe in section 2.3.1 that injection of fluid causes an increase in the pore fluid pressure of the reservoir, resulting in a decrease in the effective normal stress and contributing to the likelihood of Coulomb failure. In an isotropic media, pore fluid pressure diffuses radially outward from the borehole into the reservoir 
(Shapiro and Dinske, 2009). We have determined a range of possible hydraulic diffusivity corresponding to how the detections' distance from NM8 evolve with time. Figure 4.22 illustrates that when considering $75 \%$ of the seismicity (as depicted via the box and whisker plot for each day), the seismicity is contained within an envelope of expanding fluid pressured governed by a hydraulic diffusivity of $0.05 \mathrm{~m}^{2} / \mathrm{s}$. Considering all seismicity in the cluster near NM8 would require the diffusivity of the reservoir to be approximately $0.16 \mathrm{~m}^{2} / \mathrm{s}$ for more distant earthquakes to have occurred behind the front of pore fluid pressure diffusion. We do not necessarily require all events to be contained with in the diffusion 'envelopes' because our estimate of the diffusivity as $0.05-0.16 \mathrm{~m}^{2} / \mathrm{s}$ does not account for any possible anisotropies in the hydraulic diffusivity surrounding NM8 (Shapiro et al., 2003). By the evidence of seismicity responding to fluid diffusion, we conclude that further field events within the cluster near NM8 are induced primarily by pore fluid pressure.

\subsubsection{Coupled thermo-poroelastic mechanism}

Considering that there is evidence for seismicity induced by thermal contraction (Section 5.2.1) and by pore fluid pressure (Section 5.2.2), we accredit induced seismicity near NM8 to be caused by a coupled thermo-poroelastic mechanism. Our findings can be related to results by Martínez-Garzón et al. (2014) and Rutqvist et al. (2015), who used a combination of thermal and pore pressure perturbations to describe the mechanism of microseismicity at the Geysers geothermal field (Figure 5.3). We observe, as Martínez-Garzón et al. (2014) did, that thermoelastic stresses and poroelastic stresses may act in the same region, but at different length scales. At Ngatamariki, we observe thermal effects to act at distances less than or equal to $200 \mathrm{~m}$. We observe that poroelastic stresses induce seismicity at distances up to $2.5 \mathrm{~km}$ from well NM8 (Figure 4.22 ).

Although pore fluid pressure perturbations reach a particular area of the reservoir more quickly than thermal effects, thermoelastic stresses can still induce microearthquakes when temperature effects reach a given area. De Simone et al. (2013) constructed a conceptual model based on a coupled thermo-poroelastic mechanism of seismicity. The conceptual model shows that coupled thermalhydraulic stress can be larger in magnitude that hydraulic forces alone, partly 


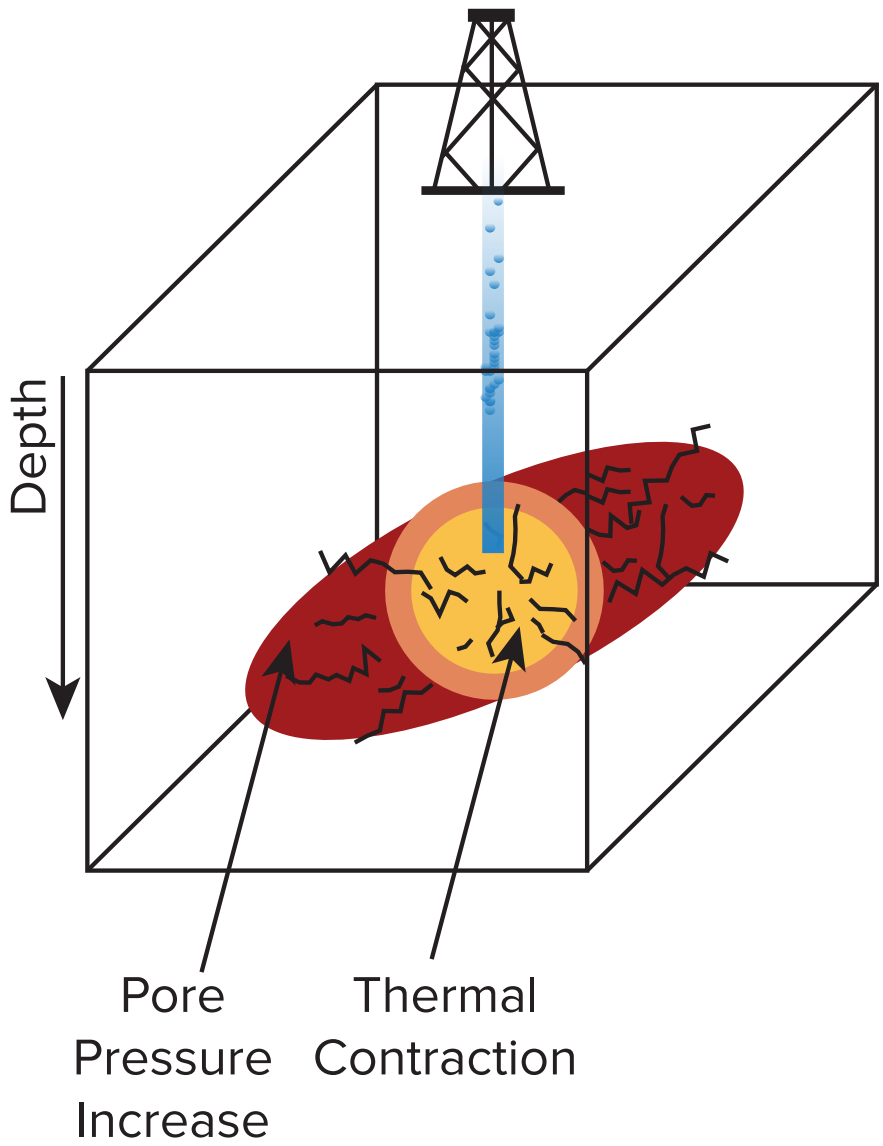

FiguRE 5.3: Diagram of thermal contraction and pore pressure increase acting at different length scales. The concentric ellipses represent the extent to which fluid/temperature has propagated The warmer ellipse color corresponds to warmer injectate temperature (Martínez-Garzón et al., 2014, adapted).

due to the lower injected fluid pressure temperature causing an increase in injectate viscosity (De Simone et al., 2013). A key concern from the conceptual model, as it relates to this study, is stress changes resulting from a thermo-poroelastic mechanism compared with stress changes from only a hydraulic mechanism. We consider a given stress state for a location within the reservoir and represent it by a Mohr circle, which is a graph of normal stress versus shear stress for orientations of a plane relative to the maximum principal stress. Figure 5.4 illustrates the difference between a change in the stress state for a point just outside of a fracture zone due to thermo-poroelastic stresses and due to hydraulic stresses alone. The model clearly shows that coupled thermo-poroelastic stresses have a greater effect on the stress field than solely hydraulic effects. Therefore, we can conclude that even with pore fluid diffusion inducing seismicity in an area before thermalhydraulic stresses act, the thermal stresses are significantly larger than hydraulic perturbations by itself, and temperature effects will be able to induce seismicity. 


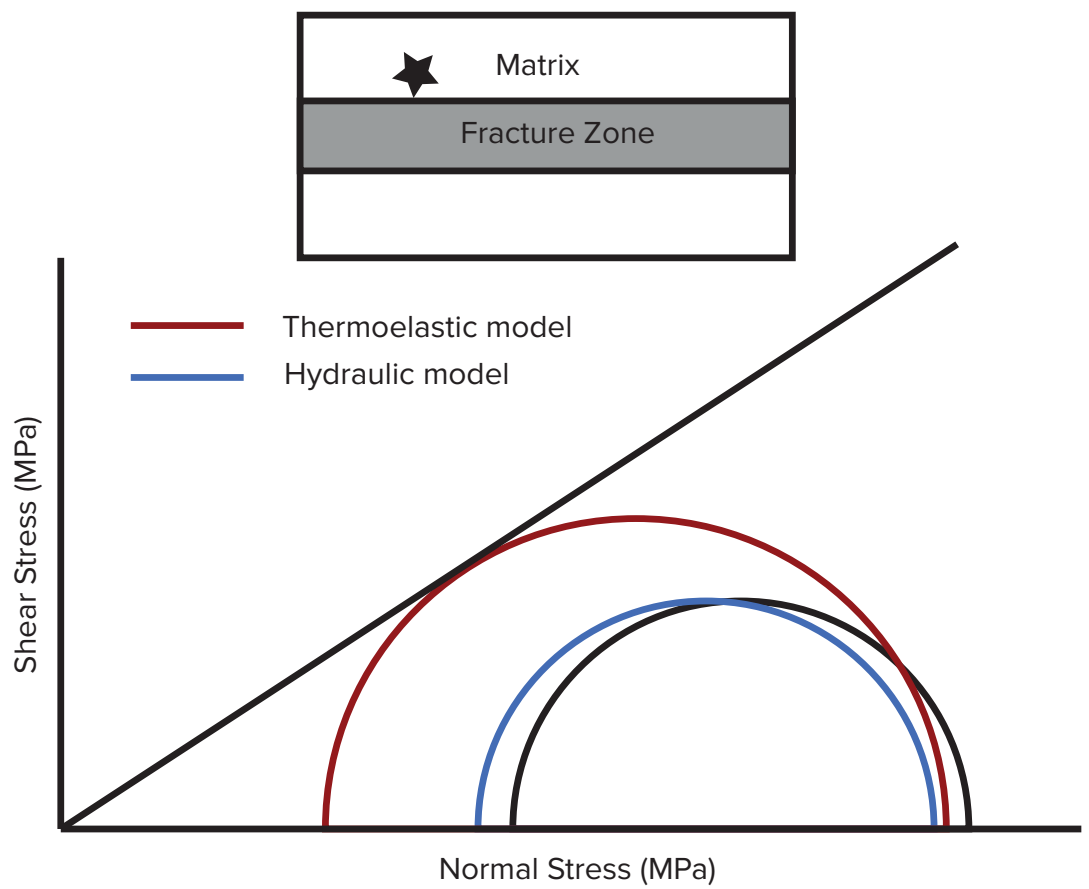

Figure 5.4: Mohr circle diagram depicting how hydraulic stresses and thermoelastic stresses change the overall stress regime at a point adjacent to the fracture network in an extensional tectonic environment ((De Simone et al., 2013 , adapted)). The point for which the stress state is being described is shown on the mini cross-section denoted by a star.

At lengths that temperature change has not yet reached, pore fluid pressure increase acts as the mechanism for seismicity, whereas closer to the injection well, temperature differences are large enough for thermoeleastic stresses to act as the mechanism for induced seismicity.

We conclude that seismicity near NM8 is induced via coupled thermo-poroelastic stresses from injection of cold water into the subsurface. We provide evidence for this mechanism by incorporating models from other studies (Bodvarsson, 1972, De Simone et al., 2013, Martínez-Garzón et al., 2014) and consider the spatiotemporal relationship with newly detected seismicity. There is still room for future work. In order to formalize the thermo-poroelastic mechanism, we recommend that other stimulation tests at Ngatamariki injection wells be considered. Additionally it would be helpful to explicitly compute the stresses that would be induced by the injected fluid at NM8. There are currently solutions that would be adaptable to Ngatamariki, including computations derived out by Rutqvist et al. (2015) and Martínez-Garzón et al. (2014). 


\section{Chapter 6}

\section{Conclusions}

We successfully apply four seismological methods to the Ngatamariki geothermal field, including (1) a matched-filter threshold determination, (2) a matched-filter methodology to detect microearthquakes within a background signal, (3) a doubledifference relocation method to obtain precise relative locations for seismicty clusters, and (4) an SVD method to determine relative magnitudes of events with highly similar waveforms. We are able to achieve two significant goals: (1) determine the viability of these methods at the Ngatamariki geothermal field and (2) characterize microseismicity at the Ngatamariki geothermal field during June--July 2012.

\subsection{Cross-correlation and matched-filtering}

We successfully apply a network matched-filtering method based on normalized waveform cross-correlation using 110 template events recorded across 11 seismic stations at the Ngatamariki and Rotokawa geothermal fields. We are able to successfully detect 862 events with $95 \%$ confidence and locate them within the field.

We apply methods based on Shelly et al. (2007) and Chamberlain et al. (2014) to determine a robust matched-filter threshold of $7.6 \times$ MAD. Our method of calculating the threshold value of 7.6 allows us to to be $95 \%$ confident that any particular detection is a true event and not a false detection. We achieve this by 
iterative synthetic testing for which we cross-correlate template events with daylong, phase-randomized noise that has been seeded at random times with template events. We determine a threshold scalar value of 7.6 for Ngatamariki by requiring all templates to detect seeds with 95\% confidence (Figure 3.9). The scalar value will vary depending study location, however we are confident in our selection of this threshold for future matched-filter studies at Ngatamariki and Rotokawa.

Although the codes used in this study are highly adaptable to other areas, we would point a potential user of this method to a more compete and maintained version of the matched-filter method: EQcorrscan (Chamberlain and Badger, 2015). The method used in this study is optimized through parallel computing in Matlab, however EQcorrscan uses parallel computing in Python, which is not limited in its computing ability in ways that Matlab is.

We do make use of EQcorrscan when calculating local earthquake magnitudes based on singular value decomposition (Chamberlain and Badger, 2015, Rubinstein and Ellsworth, 2010). Our magnitudes are limited to the seismicity cluster of greatest interest: detections located near injection well NM8. We detect events with local magnitudes as small as -0.9, though we calculate a completeness magnitude of 0.25 via maximum curvature analysis of the catalogue's Gutenberg-Richter relationship.

\subsection{Spatiotemporal analysis of newly detected seismicity}

We relocate highly-similar matched-filter detections via HypoDD, a double-difference relocation algorithm. Seismicity at Ngatamariki during June and July 2012 is distributed among three spatial clusters: seismicity near stimulated well NM8, seismicity near injection well NM10, and seismicity south of the Ngatamariki geothermal field (Figure 4.10).

These three clusters each have their own temporal signatures. Seismicity near NM8 expresses itself in bimodal manner where there are two intervals of time during which seismicity primarily occurs. From June 16-26, seismicity rate grows to a maximum of 47 earthquakes per day and fluctuates until the 26 June on which approximately 250 events have accumulated around the injection well (Figure 4.13). 
During the second interval of seismicity we see seismicity increase 4 days after injection re-commences. Additionally we also see seismicity rate decrease immediately when injection stops twice during the stimulation test period. Seismicity clusters around a depth of $2000 \mathrm{mbsl}$, approximately the location of a principal feedzone for well NM8. Earthquakes in this cluster extend to $2100 \mathrm{~m}$ from this feed zone (Figure 4.13). We conclude that the seismicity near NM8 was induced by injection of cold water during stimulation testing.

Seismicity located near injection well NM10 increases from July 15 (Figure 4.15). We see that this seismicity does not coincide with injection into NM8, but it does correlated to a period of drilling the injection well NM10. During this period of drilling we see partial and total loss of drilling fluid into the formation, coinciding with the depth and timing of seismicity near the bottom of the borehole. We conclude that seismicity near NM10 during July 2012 was induced by drilling fluid loss into the formation.

\subsection{Mechanisms of induced seismicity}

We determine that seismicity near injection well NM8 is induced by thermal stresses and pore fluid pressure increases, denoted thermo-poroelastic stresses. We show that thermal and pore fluid effects may both induce microseismicity at Ngatamariki, but on different length scales. We see temperature differences causing contraction across fractures to induce earthquakes at a scale at or less than $200 \mathrm{~m}$ from NM8, whereas seismicity exceeding $200 \mathrm{~m}$ from the well to be induced by pore fluid pressure increase.

Opportunities for future seismological work at Ngatamariki are numerous. We foresee studies that expand the methods we present and apply a matched-filter method to longer periods of time while considering seismicity during stimulation testing at NM9, NM10, and during production. We also foresee future work that focuses on mechanisms of induced seismicity, including constructing a numerical model to precisely determine a mechanism. 


\section{Appendix A}

\section{Matched-filter detection results}

TABLE A.1: Index of all template events and resulting number of detections. Template locations, times, and magnitudes were provided in a data release from Mighty River Power.

\begin{tabular}{cccccccc}
\hline Index & Date & Time & Lat & Lon & Depth $(\mathrm{km})$ & Magnitude & No. of Detections \\
\hline 1 & $3 / 06 / 2012$ & $17: 42: 13$ & -38.5804 & 176.119 & 5.76 & 0.51 & 16 \\
2 & $7 / 06 / 2012$ & $1: 59: 16$ & -38.6082 & 176.2123 & 7.45 & 2.15 & 72 \\
3 & $7 / 06 / 2012$ & $2: 42: 25$ & -38.5975 & 176.2063 & 6.2 & 1.56 & 46 \\
4 & $7 / 06 / 2012$ & $2: 53: 32$ & -38.6053 & 176.2098 & 7.12 & 1.76 & 66 \\
5 & $13 / 06 / 2012$ & $4: 02: 17$ & -38.6035 & 176.2071 & 6.2 & 1.25 & 103 \\
6 & $13 / 06 / 2012$ & $6: 32: 24$ & -38.6102 & 176.2127 & 7.1 & 1.28 & 59 \\
7 & $16 / 06 / 2012$ & $2: 44: 28$ & -38.5339 & 176.1778 & 2.13 & 0.83 & 571 \\
8 & $17 / 06 / 2012$ & $21: 25: 22$ & -38.5336 & 176.1776 & 2.14 & 0.9 & 461 \\
9 & $17 / 06 / 2012$ & $23: 34: 06$ & -38.5346 & 176.1777 & 2.00 & 1.85 & 56 \\
10 & $18 / 06 / 2012$ & $15: 58: 23$ & -38.5336 & 176.1777 & 2.15 & 1.00 & 403 \\
11 & $18 / 06 / 2012$ & $16: 09: 11$ & -38.5343 & 176.1777 & 2.11 & 0.83 & 381 \\
12 & $18 / 06 / 2012$ & $16: 43: 34$ & -38.5345 & 176.1776 & 2.06 & 0.68 & 478 \\
13 & $18 / 06 / 2012$ & $11: 08: 56$ & -38.5349 & 176.1785 & 1.91 & 1.56 & 341 \\
14 & $18 / 06 / 2012$ & $11: 14: 30$ & -38.5342 & 176.1773 & 2.28 & 1.24 & 441 \\
15 & $18 / 06 / 2012$ & $13: 11: 39$ & -38.5321 & 176.1807 & 1.63 & 0.73 & 21 \\
16 & $19 / 06 / 2012$ & $19: 37: 26$ & -38.5373 & 176.1772 & 2.23 & 0.84 & 345 \\
17 & $19 / 06 / 2012$ & $14: 36: 36$ & -38.5342 & 176.177 & 1.86 & 0.05 & 472 \\
18 & $19 / 06 / 2012$ & $17: 34: 26$ & -38.535 & 176.1782 & 1.97 & 0.64 & 234 \\
19 & $19 / 06 / 2012$ & $17: 48: 23$ & -38.5342 & 176.1773 & 2.3 & 1.61 & 69 \\
20 & $19 / 06 / 2012$ & $17: 58: 10$ & -38.5344 & 176.1786 & 1.93 & 1.32 & 76 \\
21 & $19 / 06 / 2012$ & $19: 36: 50$ & -38.5321 & 176.1787 & 1.82 & 0.12 & 154 \\
22 & $19 / 06 / 2012$ & $7: 57: 49$ & -38.5346 & 176.1765 & 1.82 & 1.14 & 408 \\
23 & $19 / 06 / 2012$ & $15: 37: 37$ & -38.5381 & 176.1774 & 2.01 & 0.63 & 148 \\
24 & $19 / 06 / 2012$ & $11: 17: 37$ & -38.6094 & 176.2013 & 6.77 & 1.24 & 65 \\
\hline & & & & & & & \\
& & & & & & \\
& & & & \\
\end{tabular}




\begin{tabular}{|c|c|c|c|c|c|c|c|}
\hline Index & Date & Time & Lat & Lon & Depth (km) & Magnitude & No. of Detections \\
\hline 25 & $19 / 06 / 2012$ & 12:09:06 & -38.5337 & 176.1786 & 2.06 & 0.45 & 392 \\
\hline 26 & $19 / 06 / 2012$ & $12: 05: 43$ & -38.603 & 176.2054 & 6.43 & 2.54 & 84 \\
\hline 27 & $19 / 06 / 2012$ & $12: 28: 28$ & -38.613 & 176.1979 & 5.94 & 0.95 & 295 \\
\hline 28 & $20 / 06 / 2012$ & $6: 29: 08$ & -38.5339 & 176.1777 & 2.09 & 0.65 & 496 \\
\hline 29 & $21 / 06 / 2012$ & $7: 50: 44$ & -38.5347 & 176.1779 & 1.97 & 0.96 & 461 \\
\hline 30 & $21 / 06 / 2012$ & $15: 15: 49$ & -38.5344 & 176.1774 & 2.17 & 0.71 & 449 \\
\hline 31 & $21 / 06 / 2012$ & $13: 16: 45$ & -38.5338 & 176.1773 & 2.13 & 0.49 & 500 \\
\hline 32 & $21 / 06 / 2012$ & 13:03:43 & -38.5339 & 176.1779 & 1.96 & 0.52 & 401 \\
\hline 33 & $21 / 06 / 2012$ & $13: 19: 23$ & -38.5342 & 176.1781 & 1.99 & 0.83 & 377 \\
\hline 34 & $21 / 06 / 2012$ & 13:29:06 & -38.5347 & 176.1782 & 1.93 & 1.03 & 384 \\
\hline 35 & $21 / 06 / 2012$ & 13:30:06 & -38.535 & 176.1777 & 2.01 & 0.67 & 340 \\
\hline 36 & $22 / 06 / 2012$ & $15: 30: 30$ & -38.5333 & 176.1781 & 1.98 & 0.01 & 127 \\
\hline 37 & $22 / 06 / 2012$ & 11:49:47 & -38.5321 & 176.1799 & 1.9 & 0.52 & 43 \\
\hline 38 & $22 / 06 / 2012$ & 15:05:04 & -38.5372 & 176.1766 & 2.19 & 1.25 & 198 \\
\hline 39 & $22 / 06 / 2012$ & $5: 52: 07$ & -38.5345 & 176.1778 & 2.03 & 1.07 & 580 \\
\hline 40 & $22 / 06 / 2012$ & 5:50:40 & -38.5348 & 176.1775 & 2.11 & 1.33 & 442 \\
\hline 41 & $23 / 06 / 2012$ & 19:33:52 & -38.531 & 176.1796 & 1.98 & 0.09 & 233 \\
\hline 42 & $23 / 06 / 2012$ & $3: 24: 52$ & -38.5386 & 176.1769 & 2.11 & 0.65 & 194 \\
\hline 43 & $23 / 06 / 2012$ & $4: 36: 44$ & -38.5384 & 176.1776 & 2.12 & 1.72 & 126 \\
\hline 44 & $23 / 06 / 2012$ & 14:01:14 & -38.5344 & 176.1777 & 2.09 & 1.33 & 407 \\
\hline 45 & $24 / 06 / 2012$ & 19:48:19 & -38.5319 & 176.1749 & 2.48 & 0.27 & 436 \\
\hline 46 & $24 / 06 / 2012$ & 12:10:56 & -38.5344 & 176.1772 & 2.13 & 0.68 & 436 \\
\hline 47 & $24 / 06 / 2012$ & 12:39:34 & -38.5345 & 176.1771 & 2.13 & 0.1 & 516 \\
\hline 48 & $25 / 06 / 2012$ & $23: 21: 47$ & -38.5337 & 176.1774 & 2.15 & 0.87 & 394 \\
\hline 49 & $25 / 06 / 2012$ & 23:38:04 & -38.5345 & 176.177 & 2.15 & 1.9 & 315 \\
\hline 50 & $25 / 06 / 2012$ & 23:30:33 & -38.5345 & 176.1782 & 1.93 & 1.28 & 316 \\
\hline 51 & $6 / 07 / 2012$ & 17:06:01 & -38.5391 & 176.1788 & 1.73 & 0.36 & 267 \\
\hline 52 & $6 / 07 / 2012$ & 18:03:04 & -38.5389 & 176.1779 & 1.57 & 0.52 & 266 \\
\hline 53 & $6 / 07 / 2012$ & 14:08:38 & -38.5343 & 176.1774 & 2.13 & 0.72 & 518 \\
\hline 54 & $6 / 07 / 2012$ & 14:27:04 & -38.5345 & 176.1775 & 2.11 & 1.49 & 414 \\
\hline 55 & $6 / 07 / 2012$ & 14:44:21 & -38.534 & 176.1775 & 2.03 & 1.07 & 281 \\
\hline 56 & $6 / 07 / 2012$ & $21: 56: 45$ & -38.5359 & 176.1763 & 1.87 & 0.2 & 520 \\
\hline 57 & $6 / 07 / 2012$ & $1: 10: 35$ & -38.5322 & 176.1782 & 1.98 & 0.73 & 483 \\
\hline 58 & $6 / 07 / 2012$ & $2: 12: 26$ & -38.5349 & 176.1777 & 2.07 & 1.05 & 463 \\
\hline 59 & $6 / 07 / 2012$ & 15:08:56 & -38.535 & 176.1779 & 2.03 & 0.58 & 487 \\
\hline 60 & $7 / 07 / 2012$ & 21:35:19 & -38.5391 & 176.1774 & 1.94 & 0.93 & 138 \\
\hline 61 & $7 / 07 / 2012$ & 22:34:12 & -38.5338 & 176.1774 & 1.97 & 0.21 & 351 \\
\hline 62 & $7 / 07 / 2012$ & 19:46:37 & -38.5374 & 176.1789 & 1.19 & 0.89 & 64 \\
\hline 63 & $7 / 07 / 2012$ & 20:20:50 & -38.5372 & 176.1791 & 1.44 & 1.26 & 34 \\
\hline 64 & $7 / 07 / 2012$ & 20:52:33 & -38.5374 & 176.1789 & 1.39 & 1.02 & 59 \\
\hline 65 & $7 / 07 / 2012$ & 22:14:48 & -38.5368 & 176.1789 & 1.49 & 1.55 & 30 \\
\hline 66 & $7 / 07 / 2012$ & 0:54:39 & -38.5389 & 176.1784 & 1.51 & 1.11 & 108 \\
\hline
\end{tabular}




\begin{tabular}{|c|c|c|c|c|c|c|c|}
\hline Index & Date & Time & Lat & Lon & Depth (km) & Magnitude & No. of Detections \\
\hline 67 & $7 / 07 / 2012$ & 1:32:48 & -38.5384 & 176.1773 & 1.84 & 1.51 & 53 \\
\hline 68 & 7/07/2012 & 9:23:56 & -38.5341 & 176.1781 & 2 & 1.02 & 548 \\
\hline 69 & $7 / 07 / 2012$ & $5: 32: 20$ & -38.5354 & 176.1782 & 2 & 0.22 & 497 \\
\hline 70 & 7/07/2012 & $5: 48: 43$ & -38.5344 & 176.1776 & 2.13 & 0.71 & 468 \\
\hline 71 & 7/07/2012 & $9: 26: 21$ & -38.5369 & 176.1775 & 1.53 & 0.48 & 132 \\
\hline 72 & $8 / 07 / 2012$ & $20: 46: 25$ & -38.5376 & 176.1775 & 1.4 & 0.86 & 57 \\
\hline 73 & $8 / 07 / 2012$ & 10:15:44 & -38.5374 & 176.1774 & 2.1 & 0.93 & 245 \\
\hline 74 & $16 / 07 / 2012$ & 21:38:08 & -38.5692 & 176.1913 & 3.03 & 0.68 & 154 \\
\hline 75 & $17 / 07 / 2012$ & 2:09:00 & -38.5614 & 176.1916 & 2.88 & 0.99 & 204 \\
\hline 76 & $17 / 07 / 2012$ & 10:31:50 & -38.5662 & 176.1891 & 3.35 & 0.78 & 196 \\
\hline 77 & $17 / 07 / 2012$ & 10:41:28 & -38.5594 & 176.1917 & 2.51 & 0.95 & 201 \\
\hline 78 & $17 / 07 / 2012$ & $17: 24: 17$ & -38.5654 & 176.1901 & 3.3 & 0.8 & 199 \\
\hline 79 & $17 / 07 / 2012$ & 9:23:06 & -38.5638 & 176.1903 & 3 & 1.34 & 166 \\
\hline 80 & $17 / 07 / 2012$ & $9: 53: 53$ & -38.5614 & 176.1915 & 2.95 & 0.88 & 167 \\
\hline 81 & $19 / 07 / 2012$ & 4:56:04 & -38.5632 & 176.1855 & 2.33 & 0.98 & 163 \\
\hline 82 & 19/07/2012 & $14: 22: 27$ & -38.5945 & 176.1908 & 5.24 & 0.51 & 159 \\
\hline 83 & $20 / 07 / 2012$ & 22:03:27 & -38.5657 & 176.1867 & 3.44 & 1.48 & 188 \\
\hline 84 & $20 / 07 / 2012$ & $14: 29: 18$ & -38.5667 & 176.1851 & 3.63 & 1.15 & 155 \\
\hline 85 & $20 / 07 / 2012$ & $23: 44: 02$ & -38.5645 & 176.1867 & 3.25 & 2.17 & 134 \\
\hline 86 & $20 / 07 / 2012$ & $16: 19: 28$ & -38.5678 & 176.1852 & 3.25 & 0.47 & 130 \\
\hline 87 & $20 / 07 / 2012$ & $18: 36: 16$ & -38.5637 & 176.1859 & 3.08 & 1.22 & 127 \\
\hline 88 & 20/07/2012 & 18:44:00 & -38.5652 & 176.1869 & 3.23 & 0.58 & 132 \\
\hline 89 & 20/07/2012 & 19:08:05 & -38.5656 & 176.1867 & 3.38 & 1.65 & 141 \\
\hline 90 & 20/07/2012 & $19: 27: 33$ & -38.5636 & 176.1855 & 2.94 & 0.32 & 142 \\
\hline 91 & 20/07/2012 & $19: 51: 16$ & -38.5674 & 176.1851 & 3.23 & 1.05 & 144 \\
\hline 92 & 20/07/2012 & $5: 12: 53$ & -38.5674 & 176.1848 & 3.2 & 1.35 & 126 \\
\hline 93 & $20 / 07 / 2012$ & $5: 46: 16$ & -38.5681 & 176.1839 & 3.21 & 1.25 & 102 \\
\hline 94 & $20 / 07 / 2012$ & $6: 20: 51$ & -38.5682 & 176.1846 & 3.29 & 1.26 & 96 \\
\hline 95 & $20 / 07 / 2012$ & $7: 06: 15$ & -38.567 & 176.1825 & 2.99 & 0.79 & 82 \\
\hline 96 & $20 / 07 / 2012$ & 10:16:00 & -38.5688 & 176.1835 & 4.6 & 1.64 & 176 \\
\hline 97 & $23 / 07 / 2012$ & $9: 47: 10$ & -38.5665 & 176.1864 & 3.2 & 1 & 124 \\
\hline 98 & $23 / 07 / 2012$ & $3: 29: 49$ & -38.5668 & 176.1861 & 3.19 & 1.41 & 122 \\
\hline 99 & $23 / 07 / 2012$ & $7: 22: 04$ & -38.5659 & 176.1858 & 3.33 & 1.46 & 130 \\
\hline 100 & $26 / 07 / 2012$ & $14: 18: 10$ & -38.5617 & 176.1891 & 2.12 & 0.62 & 123 \\
\hline 101 & $26 / 07 / 2012$ & $6: 26: 47$ & -38.5648 & 176.1869 & 3.3 & 1.6 & 137 \\
\hline 102 & $26 / 07 / 2012$ & 12:49:52 & -38.5714 & 176.1808 & 4.17 & 0.43 & 131 \\
\hline 103 & $27 / 07 / 2012$ & 11:40:06 & -38.562 & 176.1917 & 2.95 & 0.76 & 112 \\
\hline 104 & $27 / 07 / 2012$ & $14: 27: 31$ & -38.5738 & 176.1841 & 3.76 & 0.51 & 114 \\
\hline 105 & $27 / 07 / 2012$ & $17: 13: 56$ & -38.5727 & 176.184 & 3.81 & 0.93 & 123 \\
\hline 106 & $28 / 07 / 2012$ & 22:58:30 & -38.5659 & 176.1783 & 3.47 & 0.8 & 96 \\
\hline 107 & $28 / 07 / 2012$ & 11:22:10 & -38.5738 & 176.1833 & 3.78 & 0.78 & 57 \\
\hline 108 & $28 / 07 / 2012$ & $15: 45: 42$ & -38.5645 & 176.1892 & 2.84 & 0.68 & 67 \\
\hline
\end{tabular}




\begin{tabular}{cccccccc} 
Index & Date & Time & Lat & Lon & Depth $(\mathrm{km})$ & Magnitude & No. of Detections \\
\hline 109 & $28 / 07 / 2012$ & $9: 19: 29$ & -38.5644 & 176.1897 & 3.09 & 1.02 & 187 \\
110 & $28 / 07 / 2012$ & $12: 04: 09$ & -38.5618 & 176.1904 & 2.37 & 1 & 119 \\
\hline
\end{tabular}




\section{Bibliography}

Anstey, N. (1964). Correlation Techniques - a review. Geophysical Prospecting, $12(6): 355-382$.

Arehart, G., Christenson, B., Wood, C., Foland, K., and Browne, P. (2002). Timing of volcanic, plutonic and geothermal activity at Ngatamariki, New Zealand. Journal of volcanology and geothermal research, 116(3):201-214.

Baisch, S., Ceranna, L., and Harjes, H.-P. (2008). Earthquake cluster: what can we learn from waveform similarity? Bulletin of the Seismological Society of America, 98(6):2806-2814.

Barbier, E. (2002). Geothermal energy technology and current status: an overview. Renewable and Sustainable Energy Reviews, 6(1):3-65.

Bennett, T. J., Marshall, M. E., Barker, B. W., and Murphy, J. R. (1994). Characteristics of Rockbursts for Use in Seismic Discrimination. Technical report, DTIC Document.

Bertrand, E., Caldwell, T., Bannister, S., Soengkono, S., Bennie, S., Hill, G., and Heise, W. (2015). Using array MT data to image the crustal resistivity structure of the southeastern Taupo Volcanic Zone, New Zealand. Journal of Volcanology and Geothermal Research, 305:63-75.

Bibby, H., Caldwell, T., Davey, F., and Webb, T. (1995). Geophysical evidence on the structure of the taupo volcanic zone and its hydrothermal circulation. Journal of Volcanology and Geothermal Research, 68(1-3):29 - 58. doi: 10.1016/0377-0273(95)00007-H.

Bodvarsson, G. (1972). Thermal problems in the siting of reinjection wells. Geothermics, 1(2):63 - 66. doi: 10.1016/0375-6505(72)90013-2. 
Boseley, C., Cumming, W., Urzúa-Monsalve, L., Powell, T., and Grant, M. (2010). A resource conceptual model for the Ngatamariki geothermal field based on recent exploration well drilling and 3D MT resistivity imaging. In Proceedings World Geothermal Congress. International Geothermal Association.

Briechle, K. and Hanebeck, U. D. (2001). Template matching using fast normalized cross correlation. In Aerospace/Defense Sensing, Simulation, and Controls, pages 95-102. International Society for Optics and Photonics.

Brown, J. R., Beroza, G. C., and Shelly, D. R. (2008). An autocorrelation method to detect low frequency earthquakes within tremor. Geophysical Research Letters, 35(16).

Buscarlet, E., Moon, H., Wallis, I., and Quinao, J. (2015). Reservoir tracer test at the Ngatamariki geothermal field. In Proceedings 37th New Zealand Geothermal Workshop, page 9. New Zealand Geothermal Workshop.

Chambefort, I., Buscarlet, E., Wallis, I. C., Sewell, S., and Wilmarth, M. (2016). Ngatamariki Geothermal Field, New Zealand: geology, geophysics, chemistry and conceptual model. Geothermics, 59:266-280.

Chambefort, I., Lewis, B., Wilson, C., Rae, A., Coutts, C., Bignall, G., and Ireland, T. (2014). Stratigraphy and structure of the Ngatamariki geothermal system from new zircon U-Pb geochronology: implications for Taupo Volcanic Zone evolution. Journal of Volcanology and Geothermal Research, 274:51-70.

Chamberlain, C. and Badger, T. G. (2015). Eqcorrscan: Eqcorrscan v0.0.9. doi: 10.5281/zenodo.34113.

Chamberlain, C. J., Shelly, D. R., Townend, J., and Stern, T. A. (2014). Lowfrequency earthquakes reveal punctuated slow slip on the deep extent of the Alpine Fault, New Zealand. Geochemistry, Geophysics, Geosystems, 15(7):29842999.

Clacy, G. R. T. (1968). Geothermal ground noise amplitude and frequency spectra in the new zealand volcanic region. Journal of Geophysical Research, 73(16):5377-5383. doi: 10.1029/JB073i016p05377.

Clearwater, J., Azwar, L., Barnes, M., Wallis, I., and Holt, R. (2015). Changes in injection well capacity during testing and plant start-up at Ngatamariki. In Proceedings World Geothermal Congress 2015, volume 19, page 25. 
Clearwater, J., Burnell, J., and Azwar, L. (2011). Modelling the Ngatamariki geothermal system. In Proceedings New Zealand Geothermal Workshop 2011, page 6 .

Cole, J. and Lewis, K. (1981). Evolution of the Taupo-Hikurangi subduction system. Tectonophysics, 72(1):1-21.

Cook, N. (1976). Seismicity associated with mining. Engineering Geology, 10(2):99-122.

Davidson, J., Siratovich, P., Wallis, I., Graveley, D., and McNamara, D. (2012). Quantifying the stress distribution at the rotokawa geothermal field, new zealand. In Proceedings of 34th New Zealand Geothermal Workshop, pages 1921. New Zealand Geothermal Congress.

De Simone, S., Vilarrasa, V., Carrera, J., Alcolea, A., and Meier, P. (2013). Thermal coupling may control mechanical stability of geothermal reservoirs during cold water injection. Physics and Chemistry of the Earth, Parts A/B/C, 64:117126.

Dempsey, D., Clearwater, J., Kelkar, S., and Wallis, I. (2013). Validation of a coupled thermal-hydrological-mechanical model through a comparative study of shear stimulation in two geothermal environments: USA and New Zealand. In Proceedings of the 35th New Zealand Geothermal Workshop, page 8.

Dempsey, D., Kelkar, S., Davatzes, N., Hickman, S., Moos, D., and Zemach, E. (2014). Evaluating the roles of thermoelastic and poroelastic stress changes in the desert peak EGS stimulation. In Proceedings, Thirty-ninth Workshop on Geothermal Reservoir Engineering, page 13.

DeShon, H. R., Thurber, C. H., and Rowe, C. High-precision earthquake location and three-dimensional $\mathrm{p}$ wave velocity determination at redoubt volcano, alaska. Journal of Geophysical Research: Solid Earth, 112(B7).

Douze, E. and Sorrells, G. (1972). Geothermal ground-noise surveys. Geophysics, $37(5): 813-824$.

Du, W.-X., Thurber, C. H., and Eberhart-Phillips, D. (2004). Earthquake relocation using cross-correlation time delay estimates verified with the bispectrum method. Bulletin of the Seismological Society of America, 94(3):856-866. 
Freiberger, W. F. (1962). An approximate method in signal detection. Technical report, Division of Applied Mathematics, Brown University, Rovidence, Rhode Island.

Gibbons, S. J. and Ringdal, F. (2006). The detection of low magnitude seismic events using array-based waveform correlation. Geophysical Journal International, 165(1):149-166.

Glynn-Morris, T., Mclean, K., and Brockbank, K. (2011). Characterizing feed zones in geothermal fields: integrated learnings from completion testing, image logs and continuous core. In Proceedings New Zealand Geothermal Workshop 2011, page 8 .

Goforth, T. T., Douze, E. J., and Sorrells, G. G. (1972). Seismic noise measurements in a geothermal area. Geophysical Prospecting, 20(1):76-82. doi: 10.1111/j.1365-2478.1972.tb00620.x.

Grant, M. A. and Bixley, P. F. (2015). Geothermal Reservoir Engineering. John Wiley \& Sons, Ltd.

Grant, M. A., Clearwater, J., Quinão, J., Bixley, P. F., and Le Brun, M. (2013). Thermal stimulation of geothermal wells: a review of field data. In Proceedings, Thirty-Eighth Workshop on Geothermal Reservoir Engineering, page 7.

Gupta, H. K. and Rastogi, B. K. (1976). Dams and earthquakes. In Developments in geotechnical engineering, pages 228-229. Elsevier Scientific Pub. Co.

Gutenberg, B. and Richter, C. F. (1944). Frequency of earthquakes in california. Bulletin of the Seismological Society of America, 34(4):185-188.

Harjes, H.-P. (1997). Injection-induced earthquakes and crustal stress at $9 \mathrm{~km}$ depth at the KTB deep drilling site, Germany. Journal of Geophysical Research, 102(B8):18-477.

Havskov, J. and Ottemoller, L. (1999). Seisan earthquake analysis software. Seismological Research Letters, 70(5):532-534.

Healy, J., Rubey, W., Griggs, D., and Raleigh, C. (1968). The Denver Earthquakes. Science, 161(3848):1301-1310.

Kerr, R. A. and Stone, R. (2009). A human trigger for the great quake of Sichuan? Science, 323(5912):322-322. 
Knoll, P. (1990). The fluid-induced tectonic rockburst of March 13, 1989, in the "Werra" potash mining district of the GDR (first results). Gerlands Beitrage zur Geophysik, 99(6):239-245.

Langridge, R., Ries, W., Litchfield, N., Villamor, P., Van Dissen, R., Barrell, D., Rattenbury, M., Heron, D., Haubrock, S., Townsend, D., Lee, J., Berryman, K., Nicol, A., Cox, S., and Stirling, M. (2016). The new zealand active faults database. New Zealand Journal of Geology and Geophysics, pages 86-96.

Lees, J. M. (1998). Multiplet analysis at Coso geothermal. Bulletin of the Seismological Society of America, 88(5):1127-1143.

Lewis, J. (1995). Fast normalized cross-correlation. In Vision interface, volume 10, pages 120-123, Interval Research, Palo Alto CA.

Lyons, R. G. (2010). Understanding digital signal processing. Pearson Education.

Martínez-Garzón, P., Kwiatek, G., Sone, H., Bohnhoff, M., Dresen, G., and Hartline, C. (2014). Spatiotemporal changes, faulting regimes, and source parameters of induced seismicity: A case study from the geysers geothermal field. Journal of Geophysical Research: Solid Earth, 119(11):8378-8396.

Milicich, S. D., Chambefort, I., Bignall, G., and Clark, J. (2014). Overprinting hydrothermal systems in the Taupo Volcanic Zone. Geothermal Resources Council Transactions.

Mossop, A. P. (2001). Seismicity, subsidence and strain at the Geysers geothermal field. PhD thesis, Stanford University.

Norris, R., Koons, P., and Cooper, A. (1990). The obliquely-convergent plate boundary in the South Island of New Zealand: implications for ancient collision zones. Journal of structural geology, 12(5):715-725.

Petersen, T., Gledhill, K., Chadwick, M., Gale, N. H., and Ristau, J. (2011). The New Zealand national seismograph network. Seismological Research Letters, $82(1): 9-20$.

Poupinet, G., Ellsworth, W., and Frechet, J. (1984). Monitoring velocity variations in the crust using earthquake doublets: An application to the calaveras fault, california. Journal of Geophysical Research: Solid Earth, 89(B7):5719-5731. 
Price, R., Mortimer, N., Smith, I., and Maas, R. (2015). Whole-rock geochemical reference data for Torlesse and Waipapa terranes, North Island, New Zealand. New Zealand Journal of Geology and Geophysics, 58(3):213-228. doi: 10.1080/00288306.2015.1026832.

Raleigh, C., Healy, J., and Bredehoeft, J. (1976). An experiment in earthquake control at Rangely, Colorado. Science, 191(4233):1230-1237.

Rawlinson, Z. (2011). Microseismicity associated with actively exploited geothermal systems: earthquake detection and probabilistic location at Rotokawa and statistical seismic network design at Kawerau. Master's thesis, Victoria University of Wellington. Masters of Science Thesis.

Rowe, C., Aster, R., Phillips, W. S., Jones, R., Borchers, B., and Fehler, M. (2002a). Using automated, high-precision repicking to improve delineation of microseismic structures at the Soultz geothermal reservoir. Pure and Applied Geophysics, 159(1-3):563-596.

Rowe, C. A., Aster, R. C., Borchers, B., and Young, C. J. (2002b). An automatic, adaptive algorithm for refining phase picks in large seismic data sets. Bulletin of the Seismological Society of America, 92(5):1660-1674.

Rubinstein, J. L. and Ellsworth, W. L. (2010). Precise estimation of repeating earthquake moment: Example from parkfield, california. Bulletin of the Seismological Society of America, 100(5A):1952-1961.

Rutqvist, J., Dobson, P. F., Garcia, J., Hartline, C., Jeanne, P., Oldenburg, C. M., Vasco, D. W., and Walters, M. (2015). The northwest geysers egs demonstration project, california: Pre-stimulation modeling and interpretation of the stimulation. Mathematical Geosciences, 47(1):3-29.

Schaff, D. P. and Waldhauser, F. (2010). One magnitude unit reduction in detection threshold by cross correlation applied to parkfield (california) and china seismicity. Bulletin of the Seismological Society of America, 100(6):3224-3238.

Sewell, S., Cumming, W., Bardsley, C., Winick, J., Quinao, J., Wallis, I., Sherburn, S., and Bourguignon, S. (2015). Interpretation ofmMicroseismicity at the Rotokawa geothermal field, 2008 to 2012. In Proceedings World Geothermal Congress, volume 19, page 25. 
Shapiro, S. and Dinske, C. (2009). Fluid-induced seismicity: pressure diffusion and hydraulic fracturing. Geophysical Prospecting, 57(2):301-310.

Shapiro, S., Patzig, R., Rothert, E., and Rindschwentner, J. (2003). Triggering of seismicity by pore-pressure perturbations: Permeability-related signatures of the phenomenon. Pure and applied geophysics, 160(5-6):1051-1066.

Shapiro, S. A. (2015). Fluid-Induced Seismicity. Cambridge University Press.

Shelly, D. R., Beroza, G. C., and Ide, S. (2007). Non-volcanic tremor and lowfrequency earthquake swarms. Nature, 446(7133):305-307.

Sherburn, S. and Bourguignon, S. (2013). Ngatamariki Seismic Network and Seismicity - Annual Report 2012. Technical Report GNS Science Consultancy Report 2013/64, GNS Science.

Sherburn, S., Bromley, C., Bannister, S., Sewell, S., and Bourguignon, S. (2015a). New Zealand Geothermal Induced Seismicity: an overview. In Proceedings World Geothermal Congress 2015, volume 19, page 25.

Sherburn, S., Sewell, S., Bourguignon, S., Cumming, W., Bannister, S., Bardsley, C., Winick, J., Quinao, J., and Wallis, I. (2015b). Microseismicity at Rotokawa geothermal field, New Zealand, 2008-2012. Geothermics, 54:23-34.

Stern, T., Stratford, W., Seward, A., Henderson, M., Savage, M., Smith, E., Benson, A., Greve, S., and Salmon, M. (2010). Crust-mantle structure of the central North Island, New Zealand, based on seismological observations. Journal of Volcanology and Geothermal Research, 190(1):58-74.

Townend, J., Sherburn, S., Arnold, R., Boese, C., and Woods, L. (2012). Threedimensional variations in present-day tectonic stress along the australia-pacific plate boundary in new zealand. Earth and Planetary Science Letters, 353-354:47 $-59$.

Van Trees Jr, H. L., Baggeroer, A. B., Collins, L., Kurth, R., and Cruise, T. (1968). Detection and Estimation Theory. Technical Report Massachusetts Institute of Technology. Research Laboratory of Electronics. Quarterly Progress Report, no. 90, Research Laboratory of Electronics (RLE) at the Massachusetts Institute of Technology (MIT). 
Waldhauser, F. and Ellsworth, W. L. (2000). A double-difference earthquake location algorithm: Method and application to the northern Hayward fault, California. Bulletin of the Seismological Society of America, 90(6):1353-1368.

Wesnousky, S. G. (1994). The gutenberg-richter or characteristic earthquake distribution, which is it? Bulletin of the Seismological Society of America, 84(6):19401959.

Wilson, C., Houghton, B., McWilliams, M., Lanphere, M., Weaver, S., and Briggs, R. (1995). Volcanic and structural evolution of Taupo Volcanic Zone, New Zealand: a review. Journal of Volcanology and Geothermal Research, 68(1):128.

Wilson, C. J. and Rowland, J. V. (2016). The volcanic, magmatic and tectonic setting of the Taupo Volcanic Zone, New Zealand, reviewed from a geothermal perspective. Geothermics, 59:168-187.

Woessner, J. and Wiemer, S. (2005). Assessing the quality of earthquake catalogues: Estimating the magnitude of completeness and its uncertainty. Bulletin of the Seismological Society of America, 95(2):684-698.

Wohletz, K. and Heiken, G. (1992). Volcanology and geothermal energy. University of California Press Berkeley. http://ark.cdlib.org/ark:/13030/ft6v19p151/. 